FPGA-BASED COHERENT DOPPLER PROCESSOR FOR MARINE RADAR APPLICATIONS

Dissertation

Submitted to

The School of Engineering of the

UNIVERSITY OF DAYTON

In Partial Fulfillment of the Requirements for

The Degree of

Doctor of Philosophy in Engineering

By

Hamdi Eltayib Abdelbagi

Dayton, Ohio

May 2016

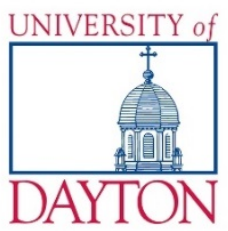


FPGA-BASED COHERENT DOPPLER PROCESSOR FOR MARINE RADAR APPLICATIONS

Name: Abdelbagi, Hamdi Eltayib

APPROVED BY:

Michael C. Wicks, Ph.D.

Advisory Committee Chairman

Ohio Research Scholar Endowed Chair in

Sensor Exploitation and Fusion;

Distinguished Research Engineer,

Department of Electrical and

Computer Engineering

Guru Subramanyam, Ph.D.

Committee Member

Chairperson of Department of

Electrical and Computer Engineering

John G. Weber, Ph.D.

Associate Dean, School of Engineering
Eric Balster, Ph.D.

Committee Member

Associate Professor, Department of

Electrical and Computer Engineering
Lorenzo Lo Monte, Ph.D.

Committee Member

Adjunct Professor, Department

of Electrical and Computer Engineering 
(C) Copyright by

Hamdi Eltayib Abdelbagi

All Right Reserved

2016 


\section{ABSTRACT \\ FPGA-BASED COHERENT DOPPLER PROCESSOR FOR MARINE RADAR APPLICATIONS}

Name: Abdelbagi, Hamdi Eltayib

University of Dayton

Advisor: Dr. Michael C. Wicks

The goal of this research is to develop a method for affordable and reliable sampling and coherent processing of measurement data collected via a modified magnetron oscillator based marine radar system. Non-coherent low-priced marine radar systems offer limited surveillance in clutter rich environments as compared to more expensive and complex coherent solid state radar systems. The approach used herein leverages modern analog to digital converters (ADC) and field programmable gate array (FPGA) technology to affordably and effectively sample the radiated and received signals for further analysis using FFT-based Doppler processing or cross correlation analysis. Track processing of moving targets is fundamental to any advanced radar and is a further focus of this research. The marine radar hardware is modified to capture the transmit signal at the source, and the receive signal at the aperture, for processing via FPGAs. The receive pulse train is cross-correlated with the transmit pulse train reference to remove the uncertainties in the phase history of the collected data. This operation ultimately makes the radar fully coherent on receive. Once the receive signal is made coherent, classical 
Doppler processing is used to differentiate moving targets from clutter and electromagnetic interference. A real time system has been built on a board with ADCs, FPGAs, and a microprocessor. Mixing of the Transmit (TX) and the Receive (RX) signals, Fourier transform analysis, and Pulse Compression are all executed digitally in the FPGA whereas Doppler Processing is performed on the microprocessor. This paper presents the underlying principles of cohering signals on receive, and it will show a realtime implementation of such algorithms using FPGAs. 
To my loving parents, my family, and my friends 


\section{ACKNOWLEDGEMENTS}

First, I would like to thank Dr. Michael C. Wicks, my advisor, for his expert guidance and encouragement. His dedication and insightful guidance have significantly contributed to the successful completion of this work.

My thanks also go to Dr. Lorenzo Lo Monte, Dr. Eric Blaster, Dr. Guru Subramanyam for helping me through my Ph.D. journey and serving as Ph.D. committee members, and also to Mansour Aljohani for his help in experimentation and data collection.

I would like to thank the Electrical and computer engineering department for offering the convenient and the suitable atmosphere to accomplish this work.

Finally, I would like to thank my parents, my brothers, my wife and my friends for their love and support. They have been a great source of inspiration and encouragement throughout my life. 


\section{TABLE OF CONTENTS}

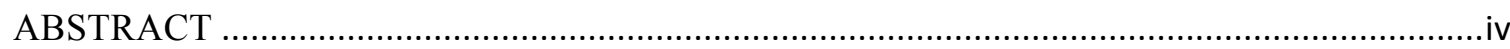

ACKNOWLEDGEMENTS ................................................................................................. vii

LIST OF FIGURES ……………………………………………………………….....

LIST OF ABBREVIATIONS AND NOTATION ....................................................................xi

CHAPTER 1 INTRODUCTION AND LITERATURE REVIEW ……………………………..... 1

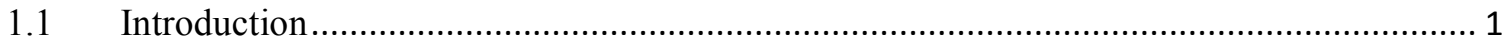

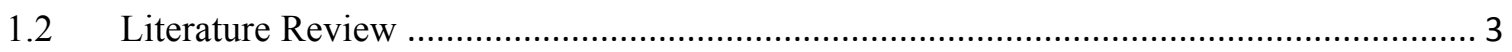

CHAPTER 2 BACKGROUND AND BRIEF DESCRIPTION OF INVENTION ........................... 5

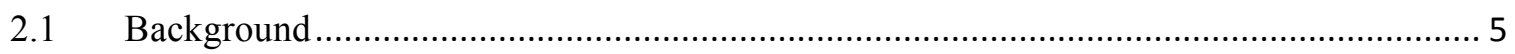

2.1.1 Non-coherent radar ......................................................................... 5

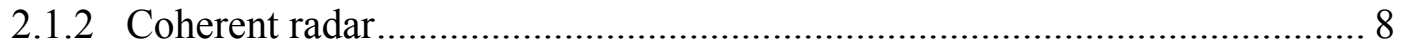

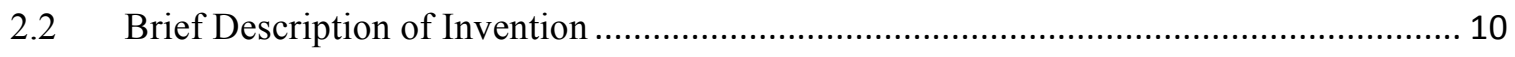

CHAPTER 3 INVENTION APPROACH ………………………………………………... 12

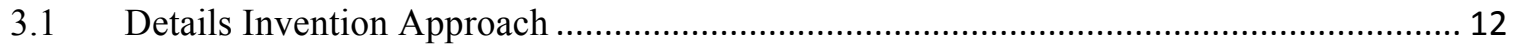

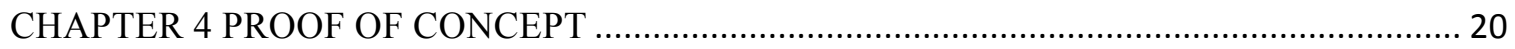

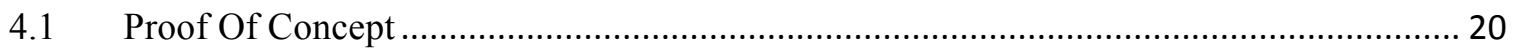

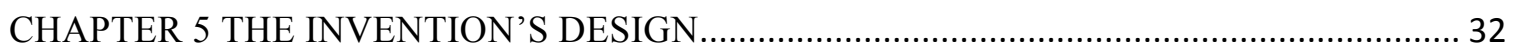

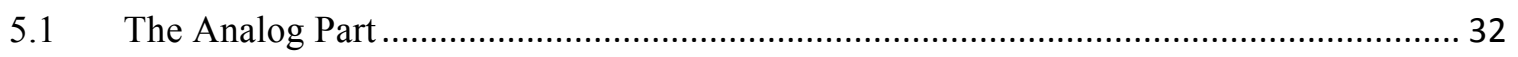

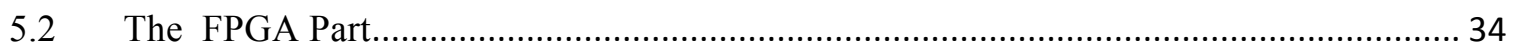

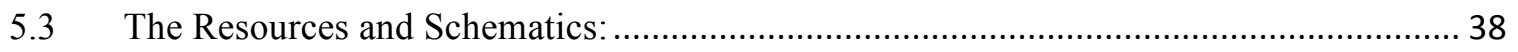

CHAPTER 6 FINDING AND RESULTS ……………………………………………. 49

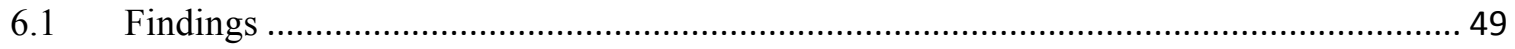

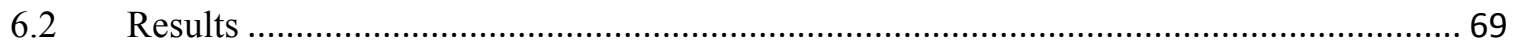

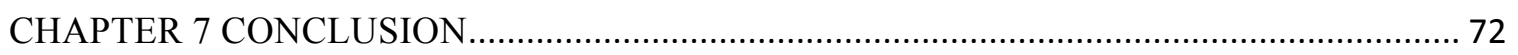

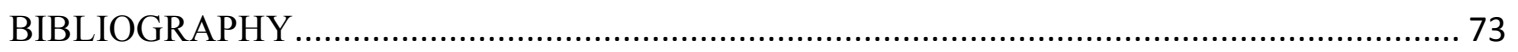




\section{LIST OF FIGURES}

Figure 1: Standard Marine Radar System ............................................................................. 5

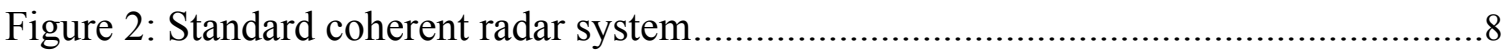

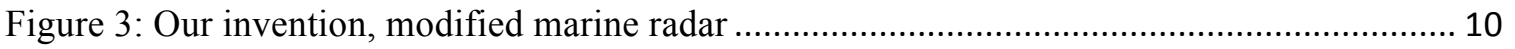

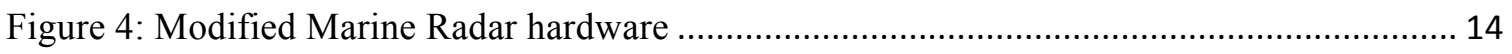

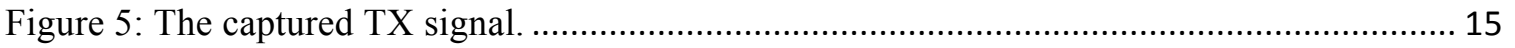

Figure 6: 2-D Data Matrix of coherent, baseband returns for M pulses and L range bins............ 17

Figure 7: FFT of the received signal to expound a Doppler shift. ............................................. 17

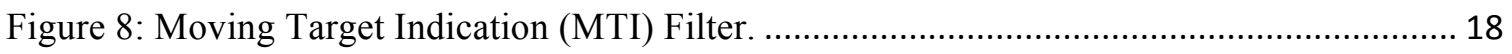

Figure 9: Doppler spectrum after Moving Target Indication filtering process. ............................ 19

Figure 10: The oscilloscope's capture for the transmitter and the receiver. ................................. 22

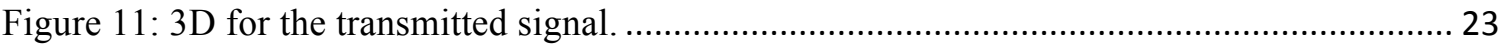

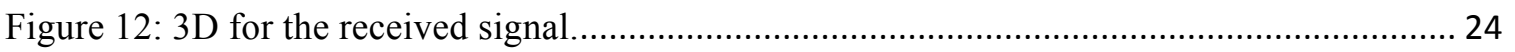

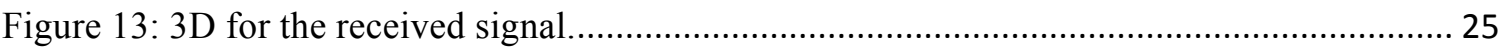

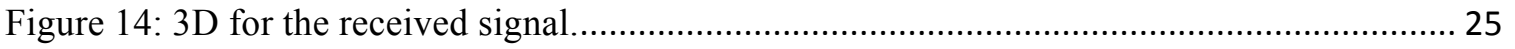

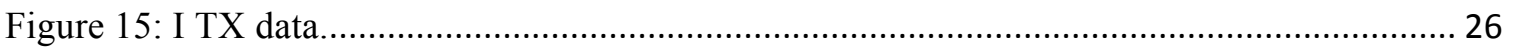

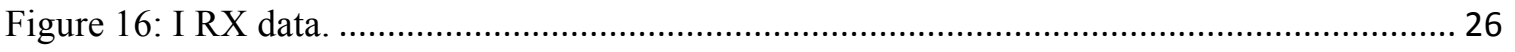

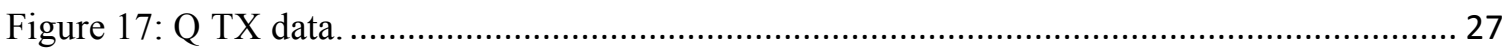

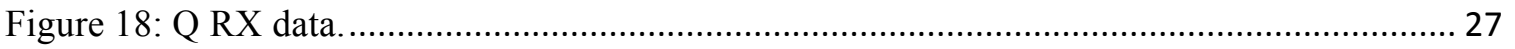

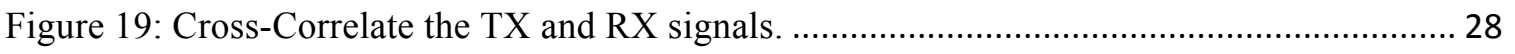

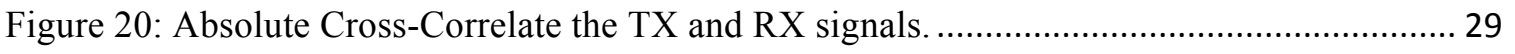

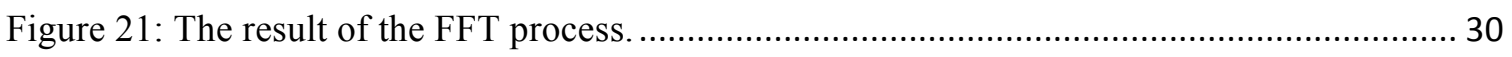

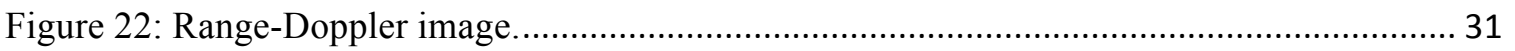

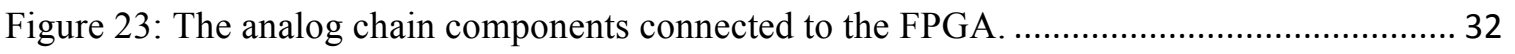

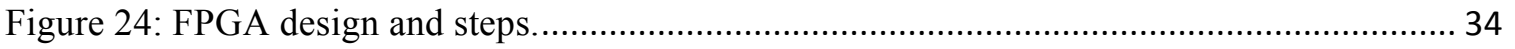

Figure 25: Marine Radar Specifications. ............................................................................ 37

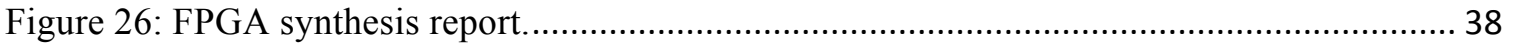

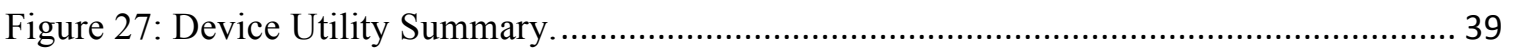

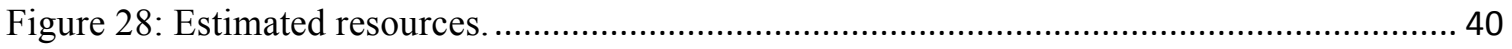

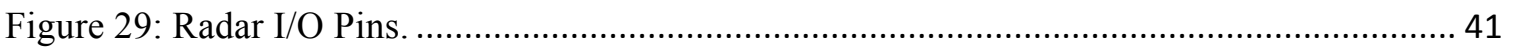

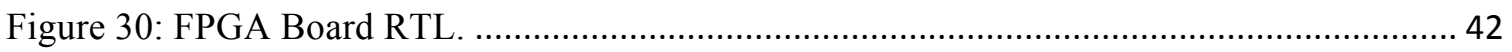

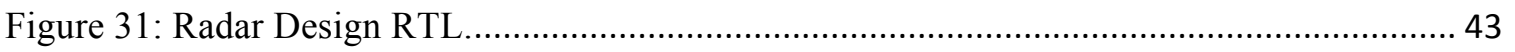

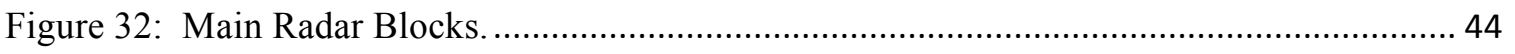

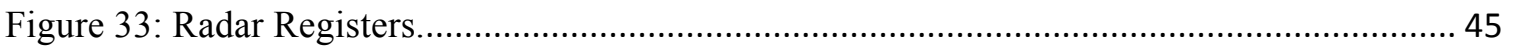




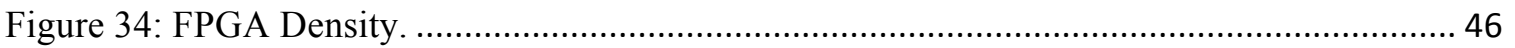

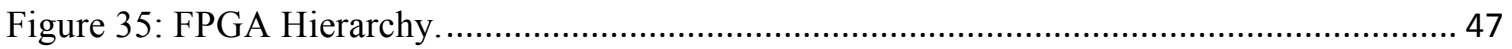

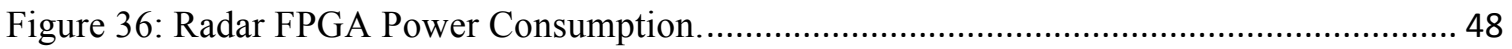

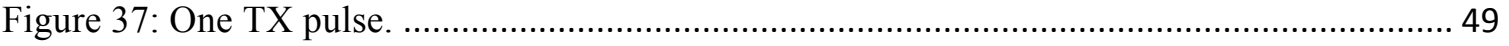

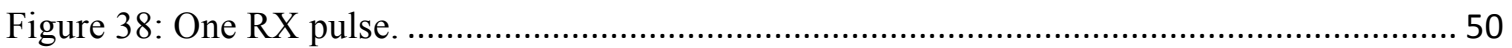

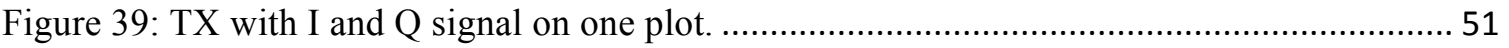

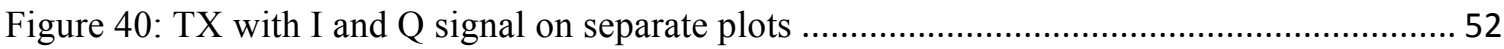

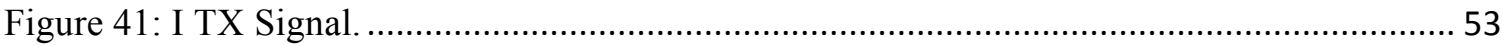

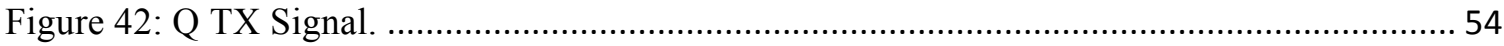

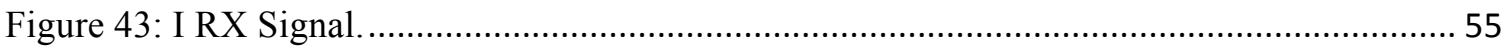

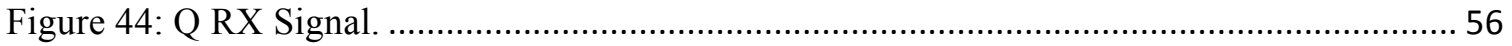

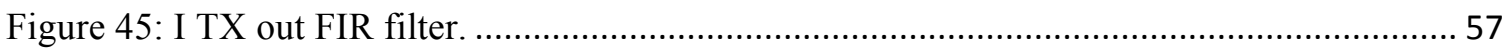

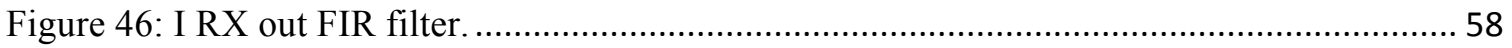

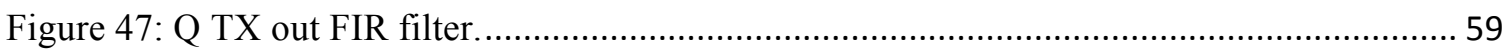

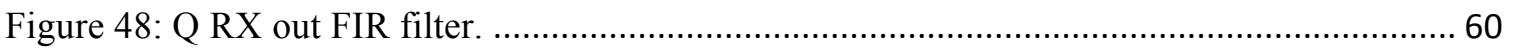

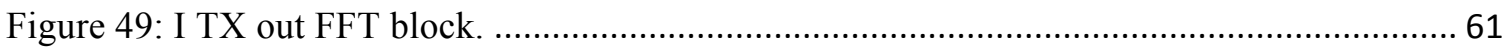

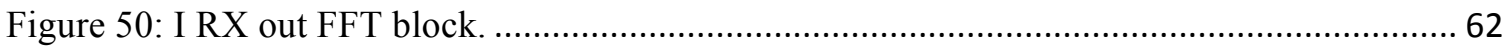

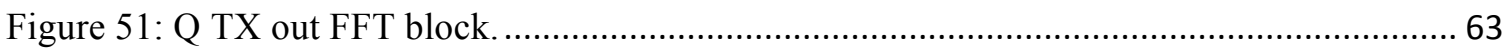

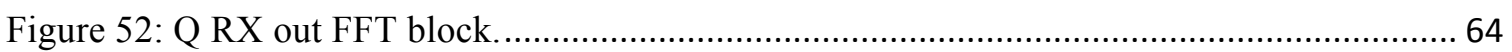

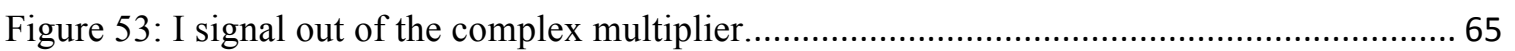

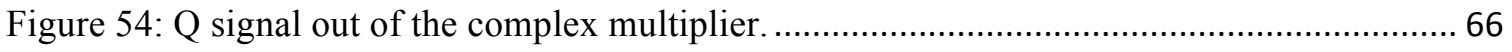

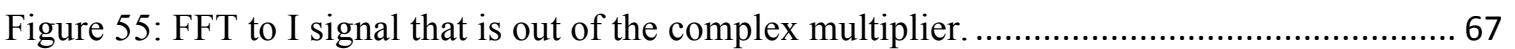

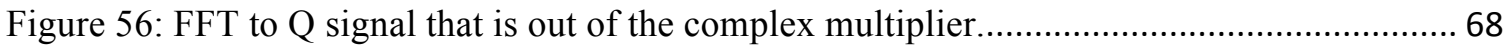

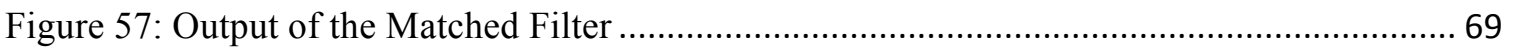

Figure 58: Range-Doppler Image Result of the FPGA design................................................. 70

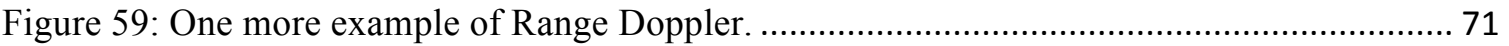




\section{LIST OF ABBREVIATIONS AND NOTATION}

\begin{tabular}{|c|c|}
\hline $\mathrm{ACP}$ & Azimuth Clock Pulse \\
\hline $\mathrm{ADC}$ & Analog-to-Digital Converter \\
\hline ADT & Automatic Detection and Tracking \\
\hline $\mathrm{AFC}$ & Automatic Frequency Control \\
\hline $\mathrm{AGC}$ & Automatic Gain Control \\
\hline AM & Amplitude Modulation \\
\hline ATC & Air Traffic Control \\
\hline ATR & Anti-Transmit-Receive \\
\hline $\mathrm{BPF}$ & Band Pass Filter \\
\hline $\mathrm{B} / \mathrm{W}$ & Black and White \\
\hline $\mathrm{CA}$ & Civil Aviation \\
\hline CFA & Crossed Field Amplifier \\
\hline
\end{tabular}




\begin{tabular}{|c|c|}
\hline CFD & Crossed Field Device \\
\hline $\mathrm{COHO}$ & Coherent Oscillator \\
\hline CRO & Cathode Ray Oscilloscope \\
\hline CRT & Cathode Ray Tube \\
\hline $\mathrm{CW}$ & Continuous Wave \\
\hline DAC & Digital-to-Analog Converter \\
\hline DSP & Digital Signal Processing \\
\hline FAR & False Alarm Rate \\
\hline FET & Field Effect Transistor \\
\hline FPF & False Plot Filter \\
\hline FTC & Fast Time Constant \\
\hline GEN & Generator \\
\hline GTC & Gain Time Control \\
\hline HPF & High Pass Filter \\
\hline IAGC & Instantaneous AGC \\
\hline ICAO & International Civil Aviation Org \\
\hline
\end{tabular}




\begin{tabular}{|c|c|}
\hline ICBM & Intercontinental Ballistic Missile \\
\hline IFA & Intermediate Frequency Amplifier \\
\hline IFF & Identification Friend or Foe \\
\hline ISLS & Interrogator Side-lobe Suppression \\
\hline LAN & Local Area Network \\
\hline LNA & Low Noise Amplifier \\
\hline LO & Local Oscillator \\
\hline LPF & Low Pass Filter \\
\hline LSB & Least Significant Bit or Byte \\
\hline LTA & Local Track Averaging \\
\hline LVA & Large Vertical Aperture \\
\hline MDS & Minimum Detectable Signal \\
\hline MIX & Mixer \\
\hline MF & Matched Filter \\
\hline MOD & Modulator \\
\hline MSB & Most Significant Bit or Byte \\
\hline MTI & Moving Target Indication \\
\hline
\end{tabular}




\begin{tabular}{|c|c|}
\hline MTD & Moving Target Detection \\
\hline MTBF & Mean Time Between Failures \\
\hline MTTR & Mean Time To Repair \\
\hline $\mathrm{OTH}$ & Over-the-horizon \\
\hline OOP & Object-Oriented Programming \\
\hline Pfa & Probability of False Alarms \\
\hline PFN & Pulse Forming Network \\
\hline PLL & Phase Locked Loop \\
\hline PPI & Plan Position Indicator \\
\hline PRF & Pulse Repetition Frequency \\
\hline PRI & Pulse Repetition Interval \\
\hline PSD & Phase Sensitive Detector \\
\hline PSR & Primary Surveillance Radar \\
\hline RAM & Random Access Memory \\
\hline $\mathrm{RCM}$ & Remote Control \& Monitoring \\
\hline $\mathrm{RCP}$ & Range Clock Pulse \\
\hline $\mathrm{RCS}$ & Radar Cross Section \\
\hline
\end{tabular}




\begin{tabular}{|c|c|}
\hline $\mathrm{RDE}$ & Radar Data Extractor \\
\hline RDP & Radar Data Processor \\
\hline RFA & Radio Frequency Amplifier \\
\hline ROM & Read Only Memory \\
\hline RPM & Revolution Per Minute \\
\hline RSL & Reduced Side Lobe \\
\hline RSP & Radar Signal Processor \\
\hline RSR & Reverse-switching Rectifier \\
\hline $\mathrm{RX}$ & Receiver \\
\hline SAM & Surface-to-Air Missile \\
\hline SAR & Synthetic Aperture Radar \\
\hline SAW & Surface Acoustic Wave \\
\hline SCR & Silicon-Controlled Rectifier \\
\hline SIF & Selective Identification Feature \\
\hline STALO & Stable Local Oscillator \\
\hline STC & Short Time Constant \\
\hline
\end{tabular}




\begin{tabular}{|c|c|}
\hline TR & Transmit-receive \\
\hline TX & Transmitter \\
\hline TWS & Track-while-scan \\
\hline TWT & Traveling Wave Tube \\
\hline VAMP & Video Amplifier \\
\hline $\mathrm{VCO}$ & Voltage- Controlled Oscillator \\
\hline FFT & Fast Fourier Transform \\
\hline IFFT & Inverse Fast Fourier Transform \\
\hline Ap & Antenna Aperture \\
\hline Av & Amplifier Voltage Gain \\
\hline B & Bandwidth \\
\hline $\mathrm{c}$ & Speed of Light \\
\hline $\mathrm{DC}$ & Direct Current \\
\hline D.C. & Duty Cycle \\
\hline $\mathrm{dB}$ & Decibel \\
\hline $\mathrm{dBm}$ & Decibel to a Milli-Watt \\
\hline $\mathrm{dBW}$ & Decibel to a Watt \\
\hline
\end{tabular}




\begin{tabular}{|c|c|}
\hline $\mathrm{Ed}$ & Magnetron Hartree Voltag \\
\hline Et & Transmitted Energy \\
\hline$f$ & Frequency \\
\hline$f \mathrm{~d}$ & Doppler Frequency Shift \\
\hline $\mathrm{G}$ & Antenna Gain \\
\hline $\mathrm{Gm}$ & Tube Trans-conductance \\
\hline $\mathrm{H}(f)$ & Transfer Function \\
\hline$h(t)$ & Impulse Response \\
\hline $\mathrm{k}$ & Boltzman's Constant \\
\hline K & Klystron Factor \\
\hline knot & Nautical Mile per Hour \\
\hline $\mathrm{n}$ & Positive Integer Number \\
\hline$\tilde{\mathrm{n}}$ & Number of Pulses \\
\hline $\mathrm{N}$ & Noise Average Power \\
\hline NF & Noise Figure \\
\hline No & Noise Density \\
\hline $\mathrm{nm}$ & Nautical Mile \\
\hline
\end{tabular}




\begin{tabular}{|c|c|}
\hline Pav & Average Power \\
\hline Pden & Power Density \\
\hline Ppeak & Peak Power \\
\hline pps & Pulse Per Second \\
\hline $\mathrm{Pd}$ & Probability of Detection \\
\hline Pfa & Probability of False Alarm \\
\hline $\operatorname{Pr}$ & Received Power \\
\hline $\mathrm{Pt}$ & Transmitted Power \\
\hline Ptx & Transmitter Power \\
\hline $\mathrm{R}$ & Slant Range \\
\hline $\mathrm{R}(\tau)$ & Autocorrelation Function \\
\hline Smin & Minimum Detectable Signal \\
\hline SCR & Signal-to-clutter Ratio \\
\hline SNR & Signal-to-noise Ratio \\
\hline $\mathrm{T}$ & Pulse Repetition Interval \\
\hline $\mathrm{Td}$ & Time Delay Elapsed \\
\hline To & Absolute Temperature \\
\hline
\end{tabular}




\begin{tabular}{|c|c|}
\hline Ts & Sampling Period \\
\hline$v$ & Velocity \\
\hline $\mathrm{Va}$ & Anode Voltage \\
\hline VA & Anode Supply Voltage \\
\hline$v \mathrm{~B}$ & Blind Speed \\
\hline $\mathrm{Vg}$ & Grid Voltage \\
\hline$v \mathrm{r}$ & Relative Velocity \\
\hline XOR & Exclusive OR \\
\hline$\alpha$ & Alpha Constant \\
\hline$\beta$ & Beta Constant \\
\hline$\beta$ & Phase Constant \\
\hline$\delta \mathrm{r}$ & Range Resolution \\
\hline$\varepsilon$ & Permittivity \\
\hline$\eta$ & Efficiency \\
\hline$\theta \mathrm{B}$ & Antenna Beam-width \\
\hline$\lambda$ & Wavelength \\
\hline & Permeability \\
\hline
\end{tabular}


$\pi$

$\sigma$

$\tau$

$\omega$

$\Phi$

$\Omega$ pi Constant

Radar Cross-Section

Pulse Duration

Angular Frequency

Phase Angle

Antenna Angular Speed 


\section{CHAPTER 1}

\section{INTRODUCTION AND LITERATURE REVIEW}

\subsection{Introduction}

Marine radars are mostly used aboard vessels for obstacle avoidance and situational realization. They are intended to show targets, including other ships, or the shoreline. Most of these targets are all stationary or very slow moving, so there will be little or no advantage in engaging fully coherent treatment in these circumstances to detect moving targets. Therefore, marine radars use non-coherent magnetron oscillator based, and hence, are inherently non-coherent. Because of the lack of coherence and phase or frequency control, these radars cannot perform Doppler processing or Moving Target Indication (MTI) filtering. Marine radars generate waveforms which match those used by coherent radars fairly well. These waveforms can be taken advantage of to make marine radars coherent. These radars are comparatively inexpensive. Non-coherent marine radars are greatly lower in cost than fully coherent solid state radars, which are intended for detecting and tracking moving targets in a dense environment. One of the main reasons for the low price of the marine radar is the fact that they use very affordable (\$30) noncoherent magnetron oscillators to generate the high power RF signal. Coherent radars require a phase locking mechanism which are fast enough to provide correct phase in the entire reception period. This technique is usually done using a quite complex phase 
locking mechanism, as well as a stable waveform within the pulse, hence increasing the requirements to expensive transmitter technology, making the fully coherent radar very expensive, and unaffordable for many end-users.

The work which has been done in this area of research is built on knowledge of how to implement coherent processing on the non-coherent signals transmitted by any source. The coherency processes in a modern radar are based upon phase-locking the local oscillator (LO) for down conversion of the RF signal. The technique showed in this paper is based on an affordable and effective technique to sample and digitize both the transmitted and received signals and matched filter them (together) for further analysis using FFT-based Doppler processing to extract the target's velocity. This technique allows for elimination of the random phase modulation on the transmit pulses, leaving only the Doppler.

The magnetron circuitry in the marine radar is modified to make it coherent by splitting the signal at the transmitter into two portions. A major portion is fed to the antenna and a smaller portion $(0.1 \%)$ to a mixer which processes the received signal by multiplying the radar echo by the transmitter leakage signal for further analysis. The transmitter leakage acts as a reference to determine the Doppler frequency change that has taken place. A similar approach has been made for correcting the phase of each transmit signal. The development of the digital signal processing and the radar practices are all taken place on the real-time system in Field Programmable Gate Array (FPGA) architecture. 


\subsection{Literature Review}

Marine radars (Furuno Marine Radar X-band) are mostly used onboard vessels for obstacle avoidance and situational alertness. They are designed to show objects on or near water, such as other ships, land or buoys. These are all stationary or slow moving targets, and there would be little benefit in employing coherent processing in this case. Marine radars, therefore, can use non-coherent transmitters such as magnetrons. The waveforms used by navigation radars (Transmit power, pulse width, pulse repetition frequency, illumination time within a beam, etc.) do however match those used by air surveillance radars quite well. Bharadwaji has proposed a waveform design for x-band radar, and later he proposed a waveform design for radar network at x-band. [7][8].

The main issue for air surveillance is the coherent processing required distinguishing small returns from moving targets and larger returns from stationary surroundings (or cluttering when looking for moving targets). Recently, X-band marine radar also utilized for marine wave observation [2], local area weather radar [3] and bird migration study [4]. In [16] is proposed to modify Furuno marine radar to handle sea clutter to increase the short range visibility of X-band marine radar.

The capability of a radar network to perform multiple radar measurements such as network attenuation correction [5] or multiple Doppler winds [6] is driven by the number of radars that have overlapping coverage at a given point, which is a function of the number of radars and the network layout.

A very similar concept to ours has been described in [9] [12], and a possible practical solution was demonstrated in [10] at Radar 2013 in the context of marine 
surveillance. The main additional contribution of this work is demonstrating Doppler range image capability, and the implementation of the real-time system in a combined FPGA/PC architecture. The principle of radar imaging was described at [11][13][14].

Radio Frequency Tomography is an imaging technology that aims to reconstruct the extended object. A network of coherent marine radar collects scattered electromagnetic field samples, which are processed to obtain $2 \mathrm{D}$ or $3 \mathrm{D}$ images of the complex dielectric permittivity profile of the volume under investigation. Unlike systems such as Synthetic Aperture Radar (SAR) or Ground Penetrating Radar (GPR) which normally employ wideband pulses, RF Tomography uses Continuous Wave (CW) signals to illuminate the scene. The information about the target is not retrieved by relying on bandwidth but by exploiting spatial, frequency and/or polarization diversity.

FPGA was adapted commercially on high-resolution range Doppler by Nicolaisen [15] And for our work, we proposed novel techniques to modify marine radar Furuno to work as a network of radar imaging processing for different applications such as border surveillance, warning tornados (e.g. tornado speed and direction) and air traffic control. 


\section{CHAPTER 2}

\section{BACKGROUND AND BRIEF DESCRIPTION OF INVENTION}

\subsection{Background}

\subsubsection{Non-coherent radar}

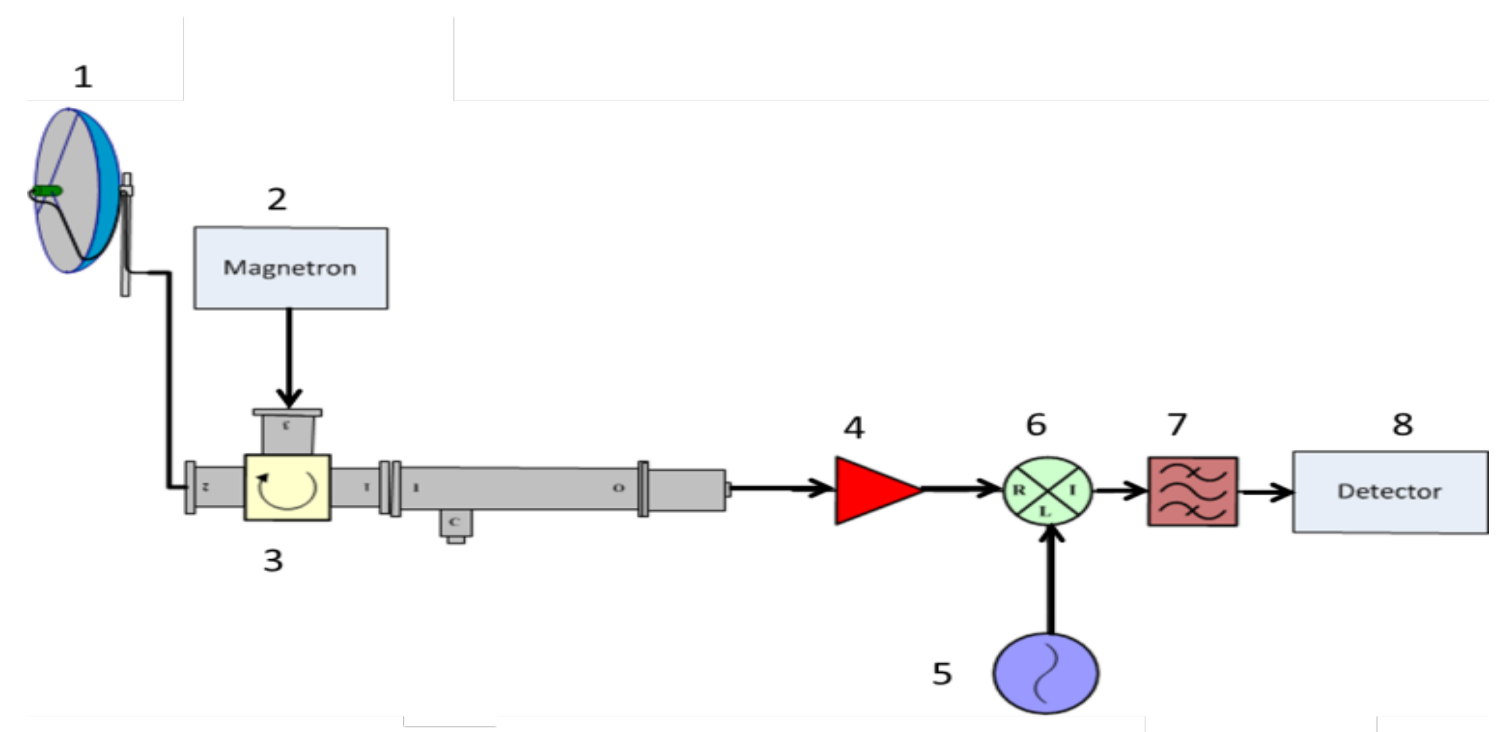

Figure 1: Standard Marine Radar System

Figure 1 shows a standard marine radar system as used on most vessels. This system employs a magnetron 2 as the transmitter source. A magnetron is a self-oscillating tube that may deliver a strong signal at a low cost. This magnetron is a random phase device, as the heating of the tube during the transmitting pulse will change its resonant frequency and shift the phase of the emitted signal. The signal from the magnetron 2 is lead to a 
scanning antenna 1 . The corresponding reflected or back-scattered signals are received by the antenna 1 and processed in a receiving channel including the components RF amplifier 4, mixer 6, a local oscillator 5, Intermediate Frequency amplifier and filter 7, and detector 8 . The detector 8 is normally a crystal (diode) amplitude detector. The signals from the detector 8 may be observed on an old fashioned Plan Projection Indicator, or digitized and observed on a raster display device as a computer screen. To protect the receiving channel from the strong transmitter signal, the signals are routed through a circulator 3 . Also, the receiving channel will be muted or turned off during the transmitting period.

To detect targets in a cluttered environment (e.g. over land), radar has to include means for extracting moving targets from the surrounding clutter. Multiple methods have been used, but may be divided into two main categories:

- Non-coherent detection where the detected video (amplitude) signal from two or more antenna scans are compared in amplitude to detect changes, thus not utilizing signal phase

- Coherent detection where signal phase changes from pulse to pulse are used to extract MTI.

The system illustrated in Figure 1 may be used for detecting moving targets, i.e. according to the first alternative, if received signals are stored during a receiving period (scan), and compared with signals received during the subsequent receiving period. 
Non-coherent moving target indication (MTI) typically is slow (as at least two complete scans of the antenna are needed for detection) and requires that the target moves in the order of one or more resolution cell within the antenna scan time. The sensitivity is also quite low because the only detected video is used.

Most modern radar systems utilize coherent detection where the signal phase from two or more pulses is compared for detection of target radial speed towards or away from the radar. These systems employ a stable oscillator that is used both for generation of the transmitter carrier frequency and for a receiver down conversion. Thus, the intermediate frequency signal in the receiver is coherent with the transmitted pulse signal, and the received signal phase depends on the target distance. A movement of the target towards or away from the radar corresponding to a fraction of a wavelength will result in a change in the received signal phase. 


\subsubsection{Coherent radar}

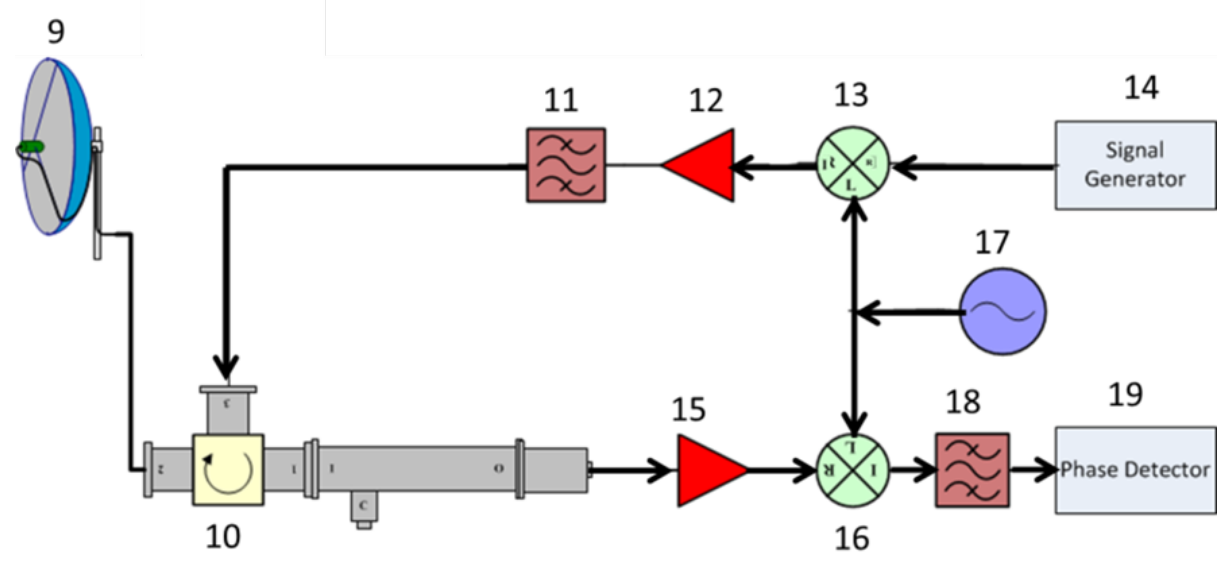

Figure 2: Standard coherent radar system

Figure 2 shows the elements of such a coherent radar system. Here radar pulses are produced in a signal generator source 14 . The generated pulses are modulating the signal from a stable carrier oscillator 17 in a mixer 13 . The transmitter pulses are then amplified in a power amplifier 12 filtered through a filter 11 and delivered to the antenna 9 via the circulator 10 . On receive, the signals are down- converted in the mixer 16 using the carrier oscillator 17 . In this way received signals will be coherent with the transmitted pulses. Subsequent pulses are compared after detection in detector 19. This system also includes an RF amplifier 15 following the circulator 10, a mixer 16 and an IF amplifier/filter 18. A moving target will show up as a phase difference between subsequent pulses. Movements as small as a fraction of the carrier wavelength may be detected. 
Construction of coherent radars is costly due to the need of phase stability in high power components. Therefore, a scheme of coherent-on-receive MTI has previously been employed, especially in high power radars. In this system, a fast local oscillator is locked to transmitter phase and frequency during the short period of transmission. After the transmitter pulse, the oscillator system is designed to hold the phase constant in the subsequent receive period. This oscillator is used for down-conversion of the received signal and thus provides coherent detection even if the transmitter has random phase. 


\subsection{Brief Description Of Invention}

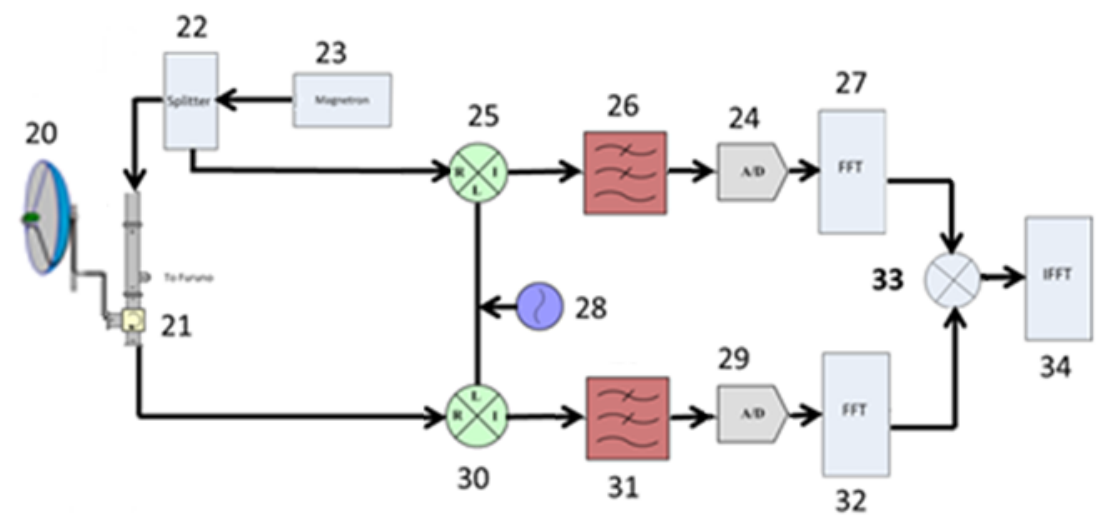

Figure 3: Our invention, modified marine radar

Figure 3 illustrates our inventive approach. This concept is based on extracting the transmit signal TX, which is generated by the magnetron 23 as a leakage signal shown in 22 , and the received signal, $\mathrm{RX}$, is sampled through circulator 21 . The RF signals and LO signals are down converted to an IF signal using the mixers 25 and 30 and then filtering these IF signal using low-pass filters 26 and 31. These signals are then digitized using A/D converters 24 and 29. The main steps are to matched filter the TX and RX signals using FFT 27 and 32, and then IFFT using 34. The result of the matched filter will be put onto slow and fast time matrix for Doppler process to extract the velocity of the target using the following equation if there is no angle between the radar and the target:

$$
D_{f}=\left(\frac{2 v}{\lambda}\right)
$$

Where $D_{f}$ is the Doppler Frequency in $\mathrm{Hz}$.

$\lambda$ is the wavelength in meter.

$v$ is the speed of the target in meter per second. 
But if the target travels in another direction of the target, the equation (1.1) becomes:

$$
D_{f}=\left(\frac{2 v}{\lambda}\right) * \cos (a)
$$

Where $\boldsymbol{a}$ is the angle between the direction of the TX/Rx signals and the direction of the target. 


\section{CHAPTER 3}

\section{INVENTION APPROACH}

\subsection{Details Invention Approach}

The X-Band Furuno DRS25A Marine Radar is magnetron oscillator based, and as such, inherently non-coherent, operating at $9410 \pm 30 \mathrm{MHz}$ with a selectable signal bandwidth of between 1.25 to $12.5 \mathrm{MHz}$. A low duty cycle gated RF pulse between $80 \mathrm{~ns}$ and $800 \mathrm{~ns}$ is employed. For traditional marine radar applications, the necessity of Doppler processing and moving target indication (MTI) is of less importance because of the subdued nature of sea clutter reflectivity, and the stationary nature of the clutter environment. Because of lacking coherence and phase or frequency control, these radars cannot perform Doppler processing or MTI filtering. Additionally, modern, coherent radars separate pulse-width control from radar resolution by employing pulse compression. It does so by transmitting frequency-modulated or phase-coded waveforms. Magnetron oscillators (pulses) do not exhibit this ability.

To exploit Doppler frequencies and detect moving targets, the radar must be fully coherent over the observation interval (number of pulses in the main beam as the radar antenna rotates by a target position). One way to do so using a magnetron oscillator based radar systems is to sample and digitize the transmitted and received signals for further analysis. 
Non-coherent marine radar systems use a magnetron to generate a pulse train. These pulse trains have no "locking" reference at the transmitter and, therefore, cannot measure Doppler. These radars only measure distance, and the speed of the target can only be determined non-coherently through radar range rate of change. In our research, the magnetron output is modified to provide a fully coherent reference. By splitting the signal from the transmitter into two portions using a sidewall coupler, the majority of which goes to the antenna, the other portion is mixed with the received signal (digitally). By multiplying the echo signal at frequency $\mathrm{fc} \pm \mathrm{fd}$ with the transmitter leakage signal at frequency fc for comparison, Doppler is computed. The sampled transmitter signal (sidewall coupler) acts as a reference to determine the target's Doppler frequency shift. 


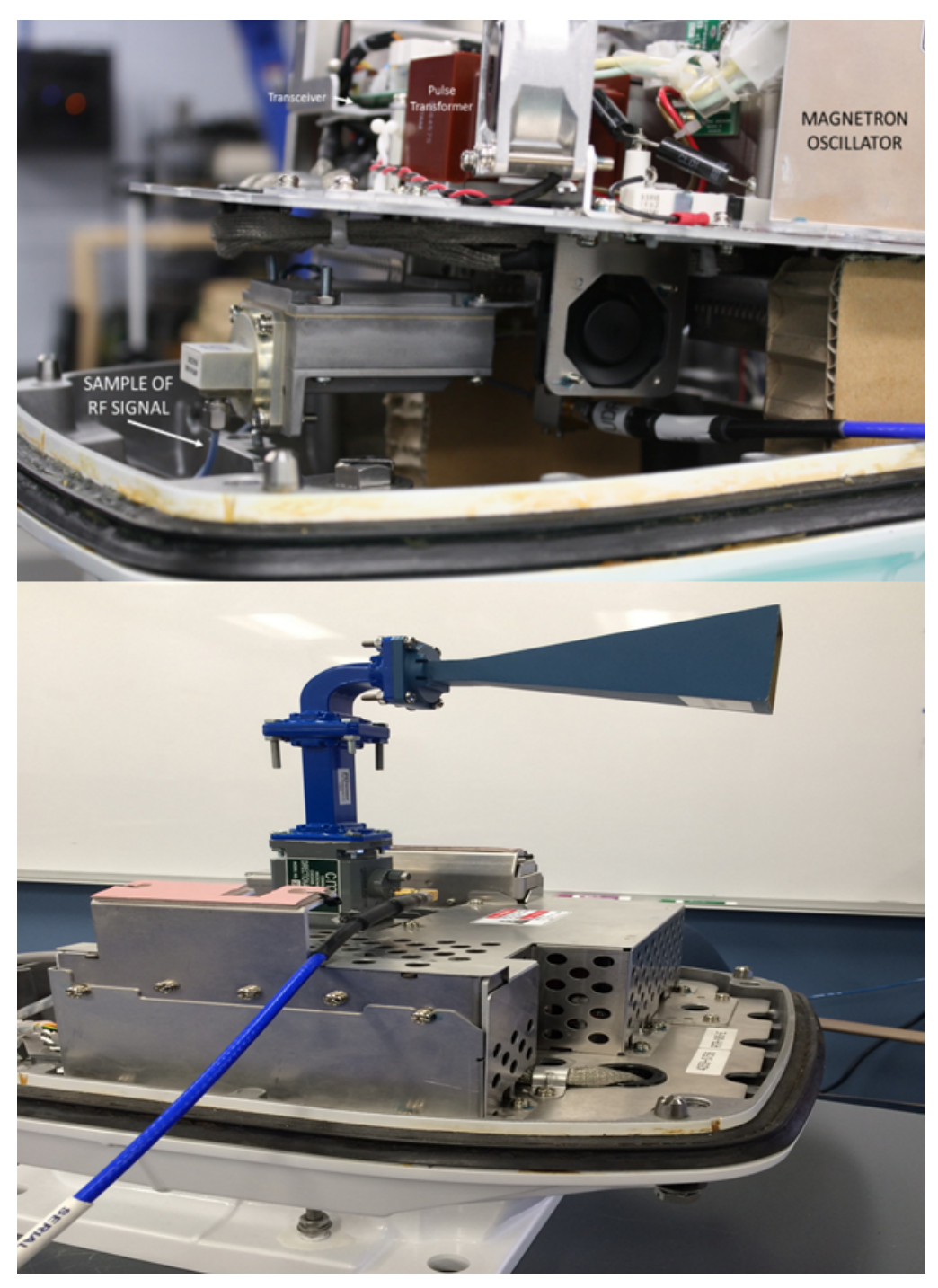

Figure 4: Modified Marine Radar hardware 


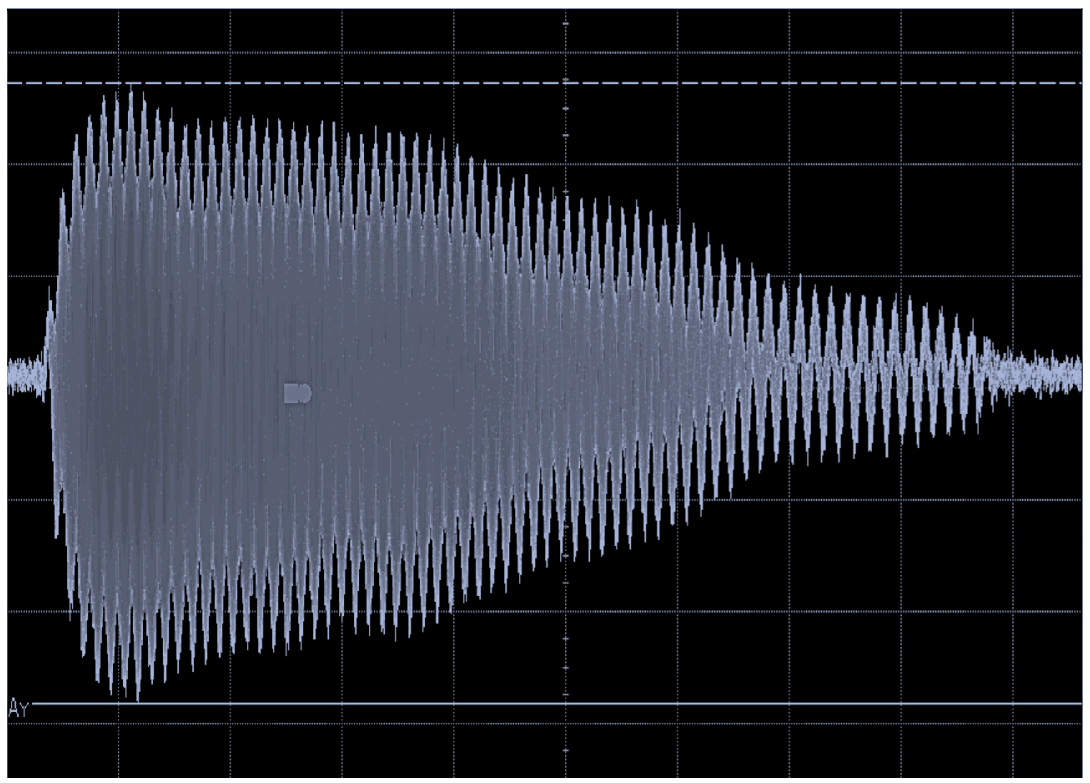

Figure 5: The captured TX signal.

Figure 4 shows the modified marine radar. Figure 5 shows a sample of the transmit signal obtained from a CMC Directional Coupler A786B and captured via a Keysight DSO-X 92004Q oscilloscope. Knowledge of the phase of the transmit signal allows the radar to differentiate between stationary clutter returns and the moving targets by correlating the transmitted and received signals. If there is any time-varying difference in phase, that will result in a Doppler shift indicating target motion.

To sample the transmit and the receive signals, the marine radar hardware is modified to access the transmit signal at the magnetron oscillator waveguide output. After capturing both the transmit and the receive signals, the next step is to down convert these signals to an intermediate frequency (IF) and digitize at a suitable sampling frequency approximately $250 \mathrm{MHz}$ in our experiment. After digitalizing these signals, the next step is accomplished via FPGA-based processing. Doppler decomposition and statistical signal processing are accomplished on FPGA boards. 
We process 100 slow time samples (pulses) or received data. With 500 samples, the SNR increases by $13 \mathrm{~dB}$. The sampled transmit and receive signals are cross-correlated via matched filtering on the FPGA board. The results of this cross-correlation processing are discussed below.

The range interval includes 2000 cells (fast time index). The data stream is decomposed into a matrix (2000 range bins x 200 pulses). Figure 3 illustrates how the 200 pulses (slow time index) can be decomposed into a 2D data matrix. Each column represents a range profile matrix (large). The fast time rate is typically $10 \mathrm{MS} / \mathrm{s}$ to $1 \mathrm{GS} / \mathrm{s}$. The first few rows in Figure 3 represent the localized source "characterization" matrix (small). The slow time rate is $100 \mathrm{~Hz}$ to $100 \mathrm{KHz}$. We integrate against thermal noise with power density $\mathrm{N}(\mathrm{N}=\mathrm{KTB})$ in the source characterization matrix. 


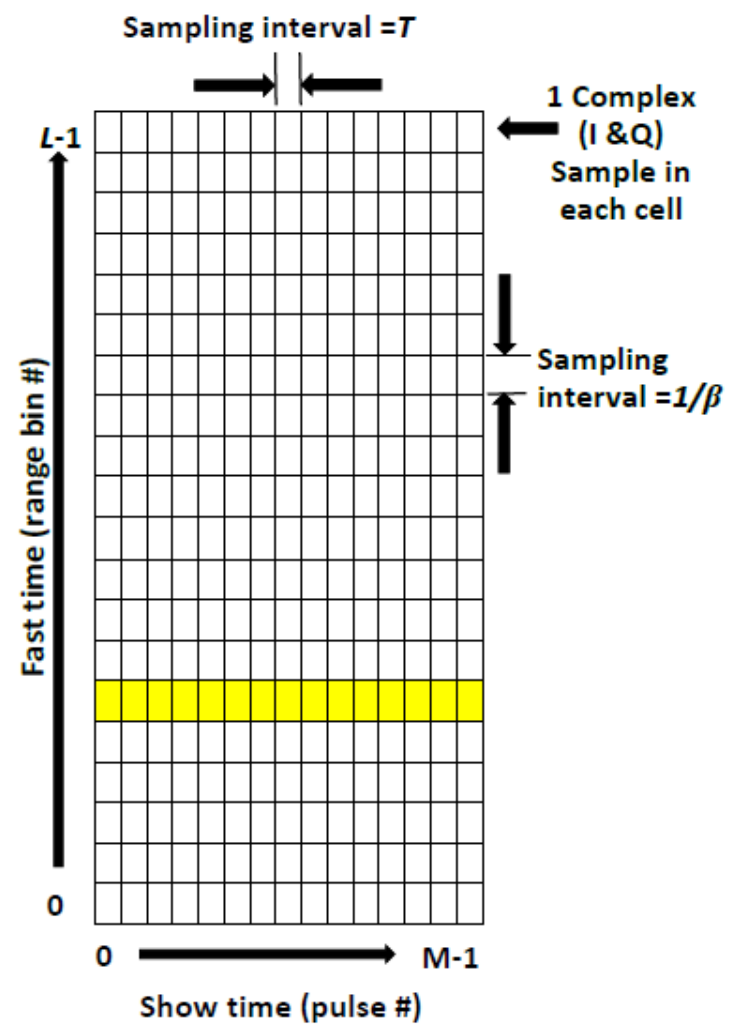

Figure 6: 2-D Data Matrix of coherent, baseband returns for $M$ pulses and L range bins.

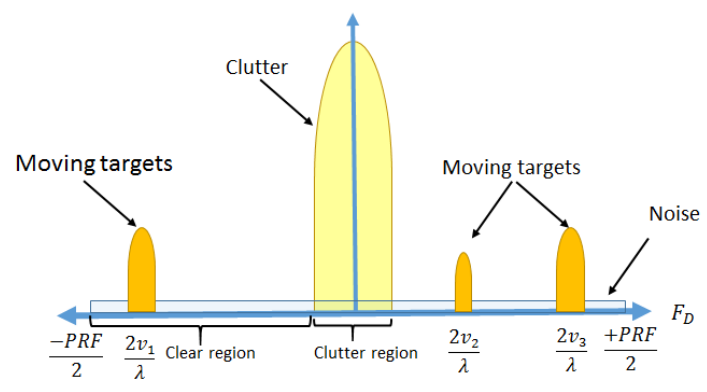

Figure 7: FFT of the received signal to expound a Doppler shift.

Figure 6 shows fast and slow time 2D matrix. 
Coherency is required to compute the target's velocity and is accomplished via Fast Fourier Transform (FFT) in slow time. Figure 7 illustrates the result of pulse Doppler processing permits visualization of target and clutter returns.

MTI applies a linear filter in the time domain to distinguish between moving targets and clutter. The concept of MTI is presented Figure 8 and in Figure 9.

MTI applies a high pass filter to the data in slow time. A filtered output retains noise and moving target returns, but reduces clutter power. MTI can only indicate whether a moving target is present or not and doesn't determine precise velocity nor the presence of multiple targets. Some of the advantages of MTI are simplicity, light computational load, and ease of implementation.

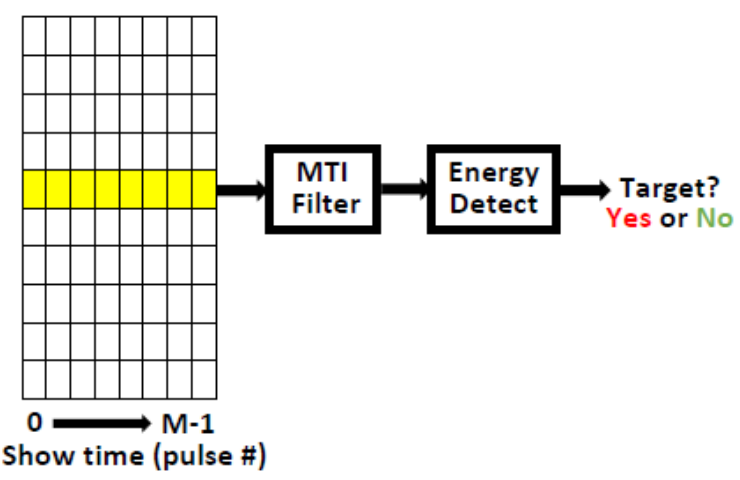

Figure 8: Moving Target Indication (MTI) Filter. 

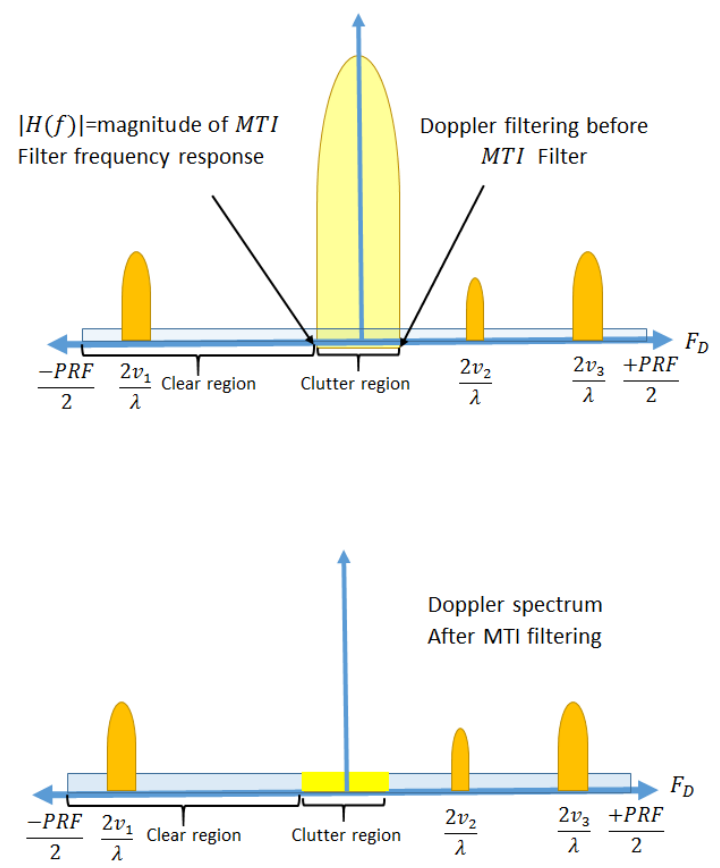

Figure 9: Doppler spectrum after Moving Target Indication filtering process. 


\section{CHAPTER 4}

\section{PROOF OF CONCEPT}

\subsection{Proof of Concept}

After we had captured the TX and RX signals, we used the MATLAB software to prove the concept of the invention before we design the real-time FPGA.

As we mentioned before that the magnetron generates random transmit phase, that is why the marine radar is non-coherent. The main idea here is to prove that the matched filter can be done independently of the random transmit phase. The following equations prove this concept:

Let's consider the transmit signal to be

$$
T(t)=x(t) e^{\left(j \omega_{c} t\right)} e^{(j \phi)}(1.2)
$$

Where $\mathrm{x}(\mathrm{t})$ is the pulse envelope.

$\omega_{c}$ is the carrier frequency.

$\emptyset$ is a random phase offset introduced by the magnetron.

Now the receive signal from the target is

$$
R(t)=x(t-\tau) e^{\left(j \omega_{c} t\right)} e^{(j \phi)} e^{\left(j \omega_{c} \phi\right)}(1.3)
$$


Where $\tau=2 \mathrm{R} / \mathrm{c}$ is the round trip delay to the target

$c$ is the speed of the light.

Now down-converting (1) and (2) with LO tuned to $\omega_{c}$ removes the $e^{j \omega_{c} t}$ from each baseband signals giving

$$
T^{\prime}(t)=x(t) e^{j \varnothing}(1.4)
$$

And

$$
R^{\prime}(t)=x(t-\tau) e^{(j \phi)} e^{\left(j \omega_{c} \tau\right)}(1.5)
$$

Next, we matched filter (3) using (4) as the filter coefficient, and this process is equivalent to cross-correlation, and the constant terms can be removed outside of this operation

The $e^{j \emptyset}$ and $e^{-j \emptyset}$ cancel leaving

$$
T^{\prime}(t) \otimes R^{\prime}(t)=x(t-\tau) e^{\left(j \omega_{c} \tau\right)} \otimes x(t)(1.6)
$$

Which is independent of the random transmit phase and equivalent to the output of the matched filter of the conventional coherent radar.

We successfully modified marine radar hardware and capture the transmitted and the received signals for coherent processing. Figure 10 shows the transmitted signal after being captured on the oscilloscope. 

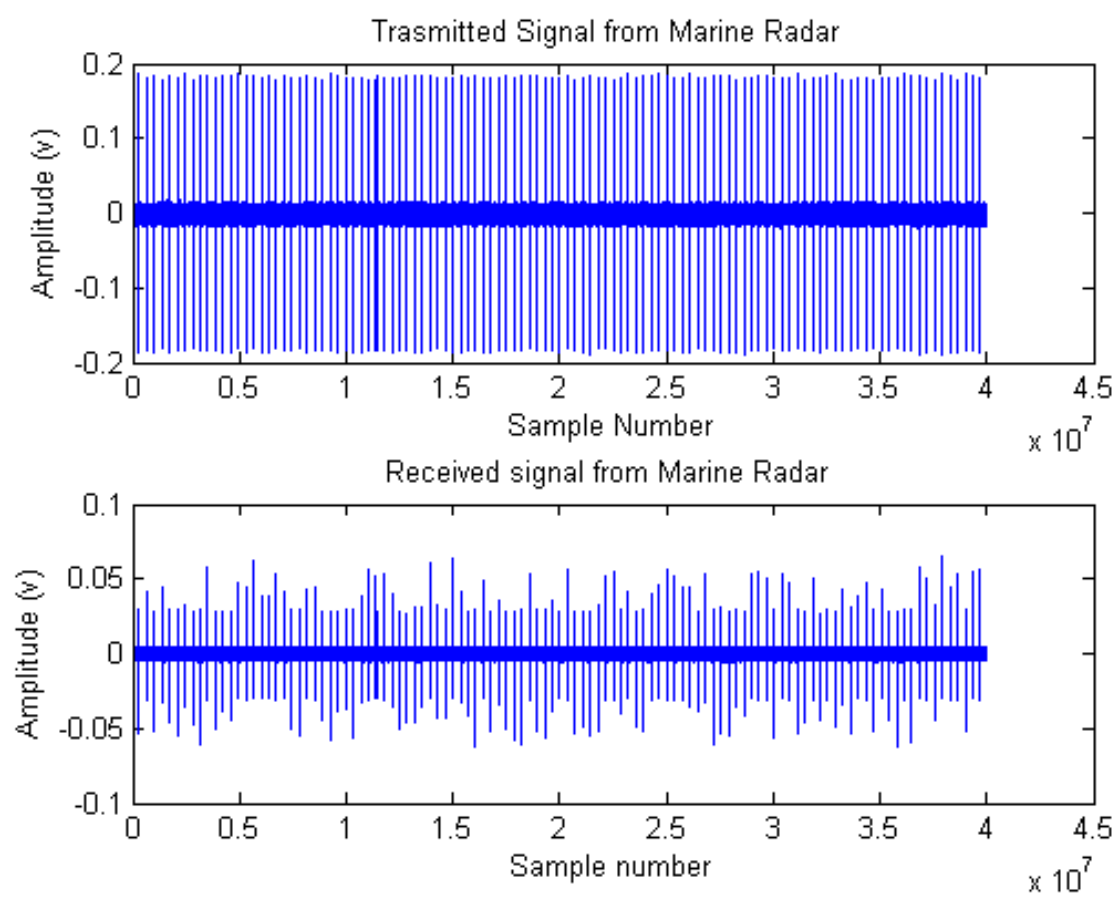

Figure 10: The oscilloscope's capture for the transmitter and the receiver.

The following steps have been taken to prove the concept of the invention.

\section{A. Capturing the TX and RX signals}

After modifying the hardware marine radar, we captured the TX and the RX signals. The following plots show the TX and RX signals in a $2 \mathrm{D}$ image. 


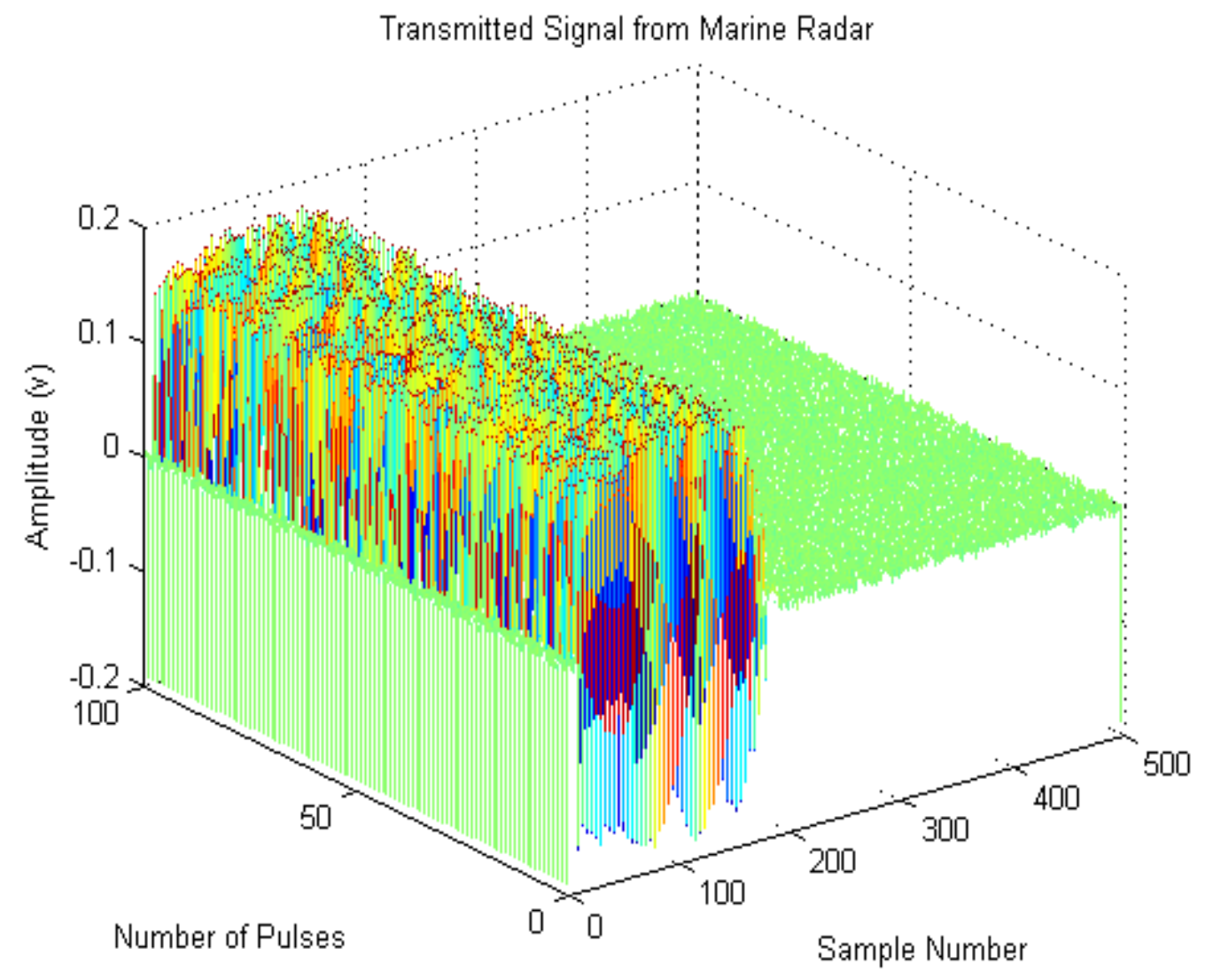

Figure 11: 3D for the transmitted signal. 


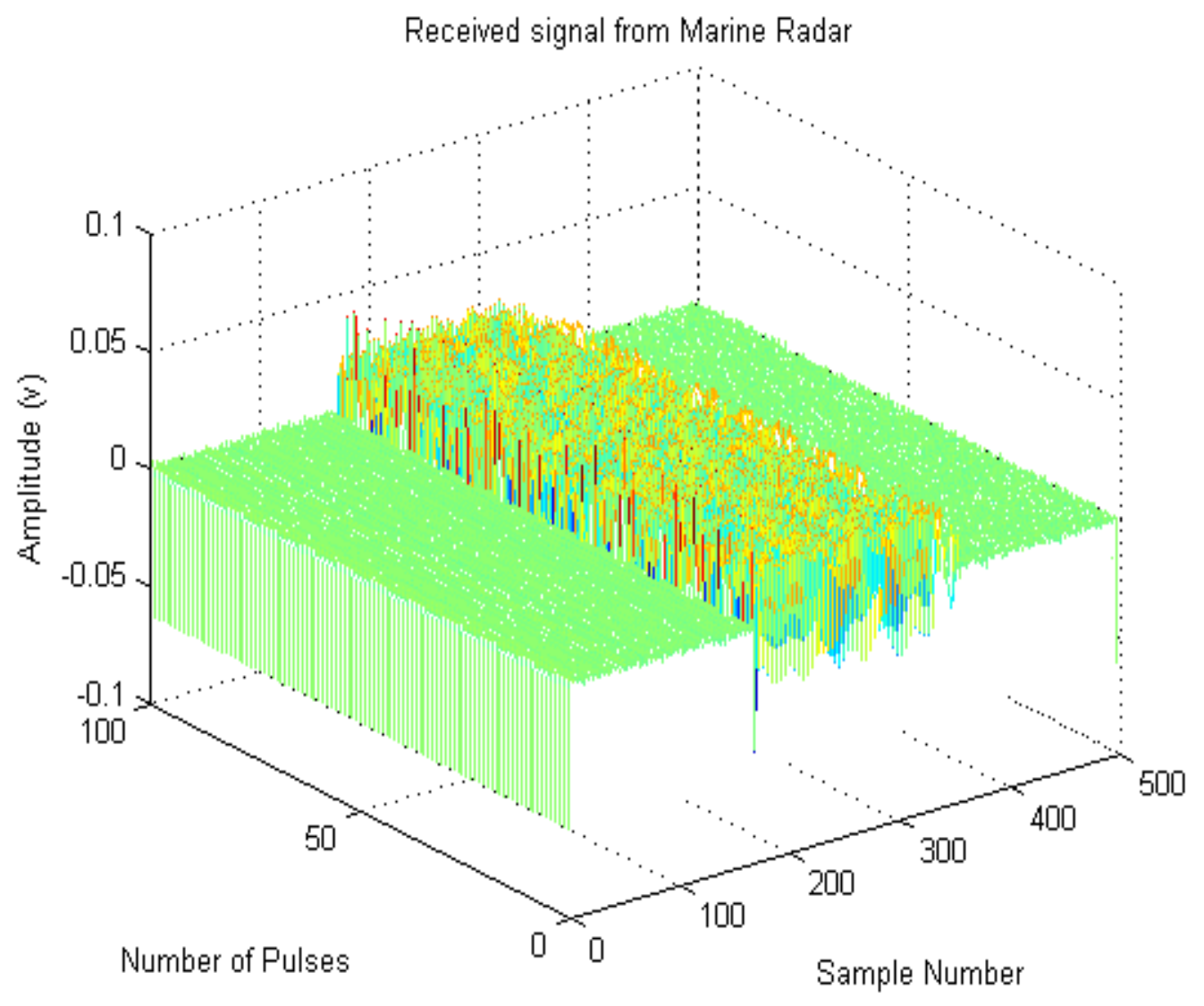

Figure 12: $3 D$ for the received signal. 


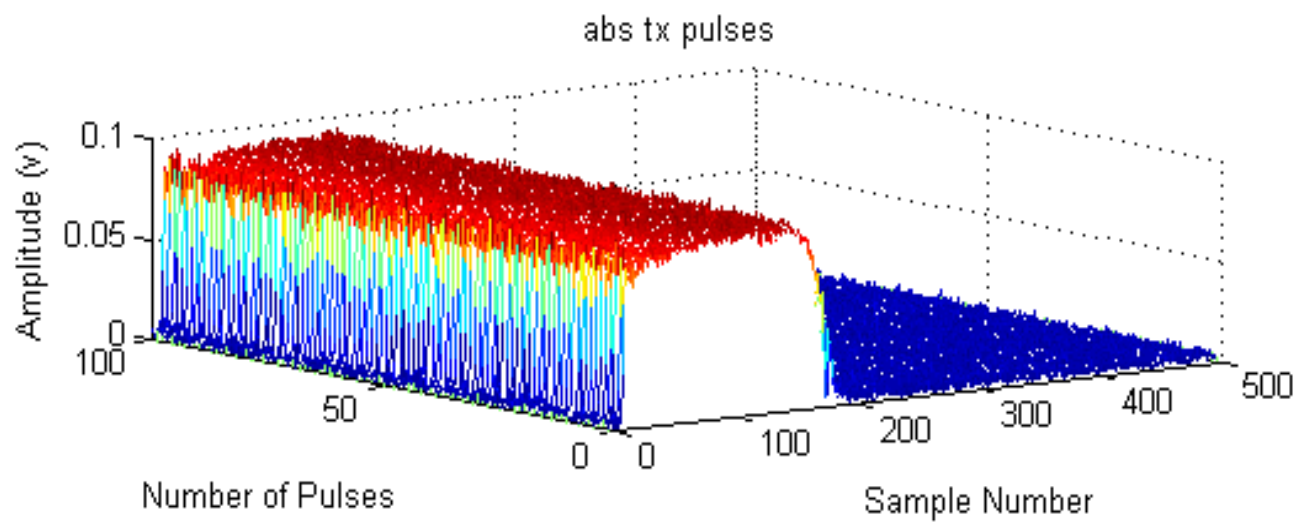

Figure 13: 3D for the received signal.

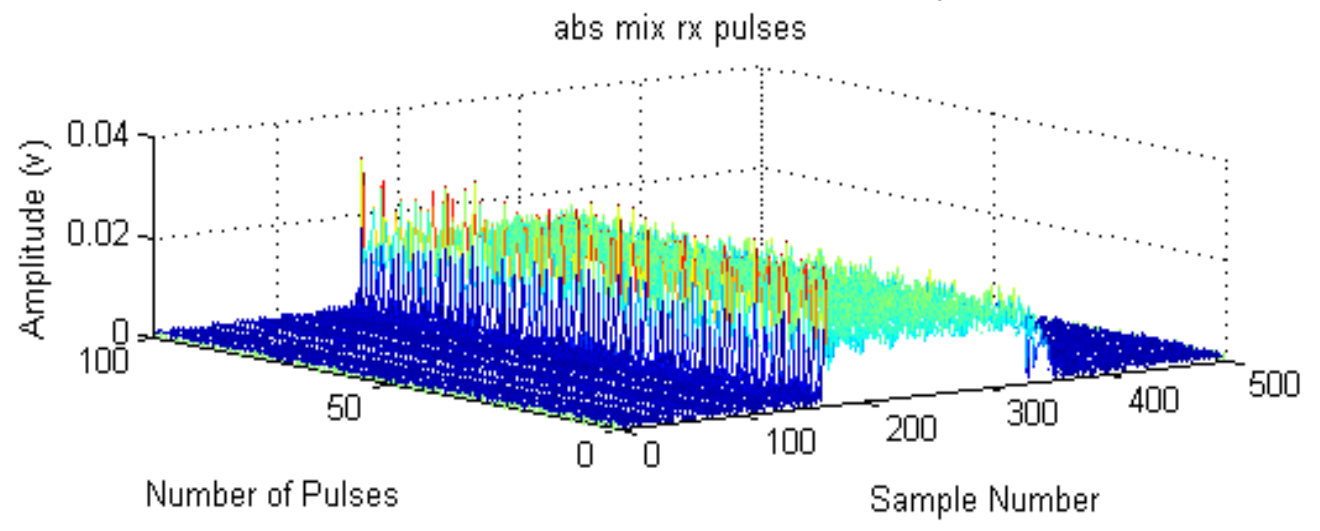

Figure 14: 3D for the received signal. 


\section{B. Mixing RF signal to get IF, then get reduced to I-Q.}

The RF signal got mixed to IF signal using $50 \mathrm{MHz}$ LO. The following plots show I TX, I RX, Q TX, and Q RX respectively.

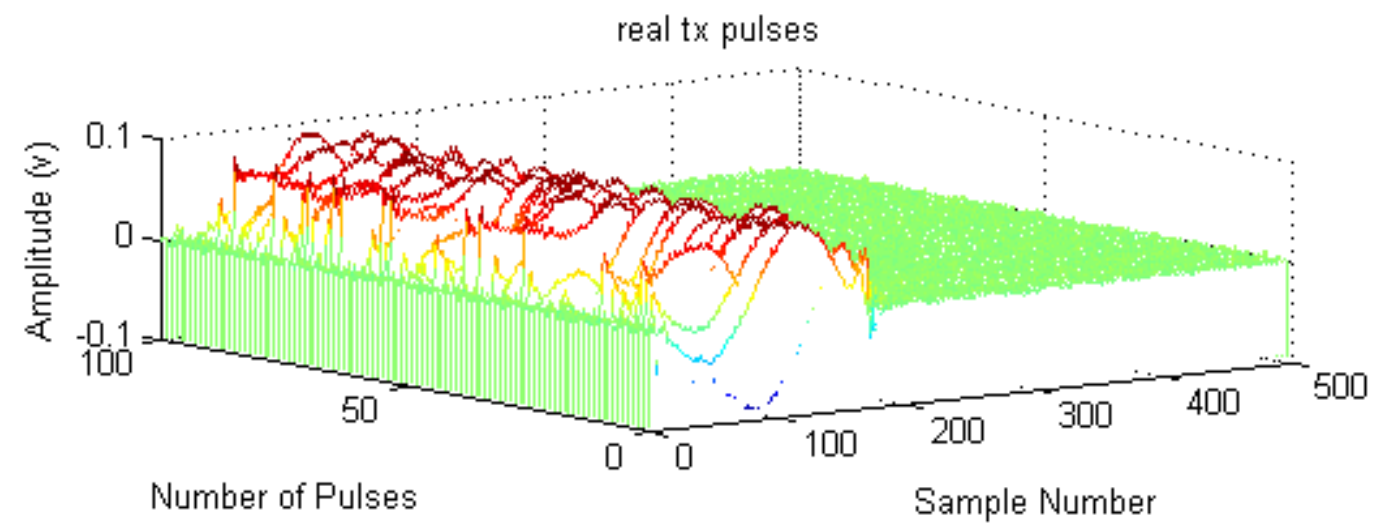

Figure 15: I TX data.

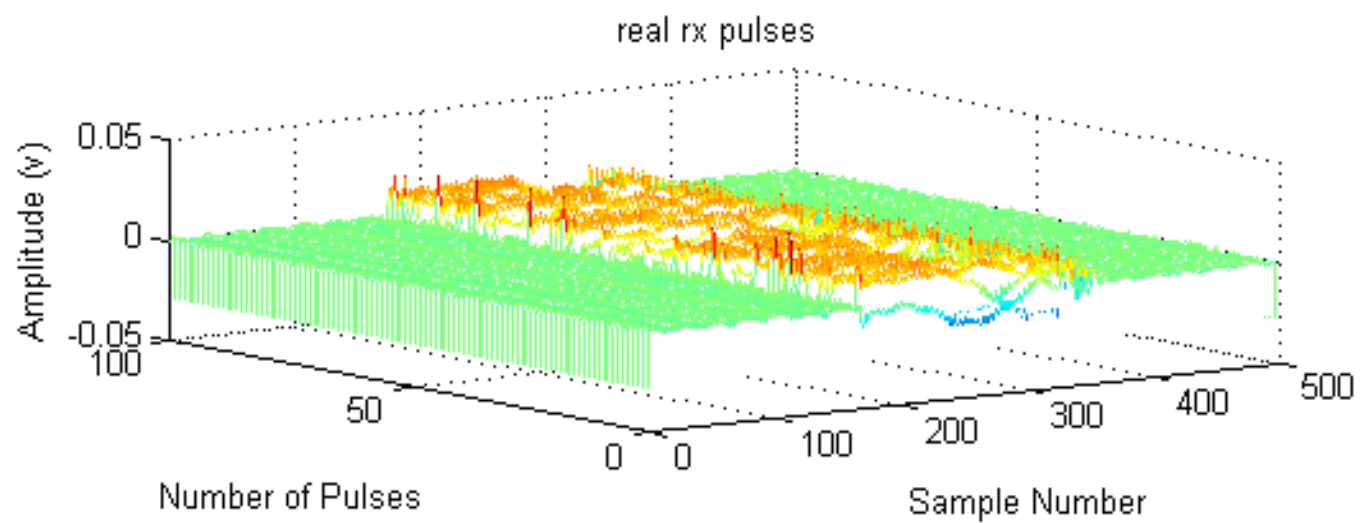

Figure 16: I RX data. 


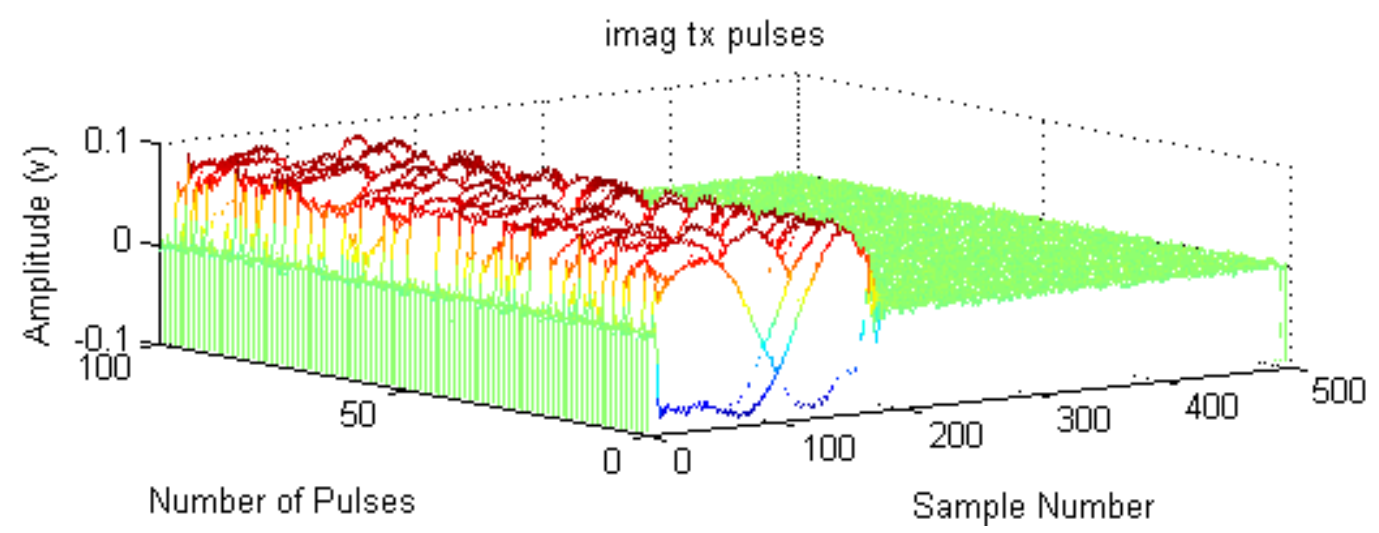

Figure 17: Q TX data.

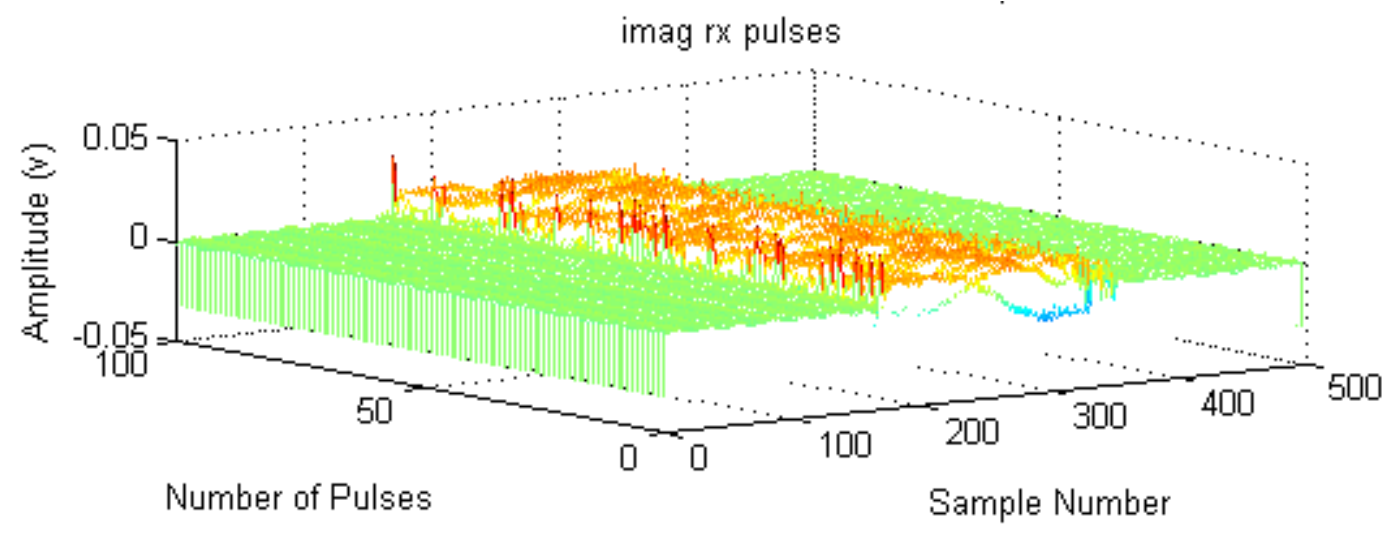

Figure 18: $Q R X$ data. 


\section{Matched Filter (cross-correlation)}

After we performed all the basic processing of the TX and RX data, we apply radar algorithms such as matched filter (cross-correlation) processing on same data.

The correlation is standard cross-correlation product:

$$
r_{x y}(l)=\sum_{n=-\infty}^{\infty} x(n) y(n-1) \quad l=0, \pm 1, \pm 2, \ldots
$$

Figure 19 and Figure 20 show the result of cross-correlation of the TX and RX signals.

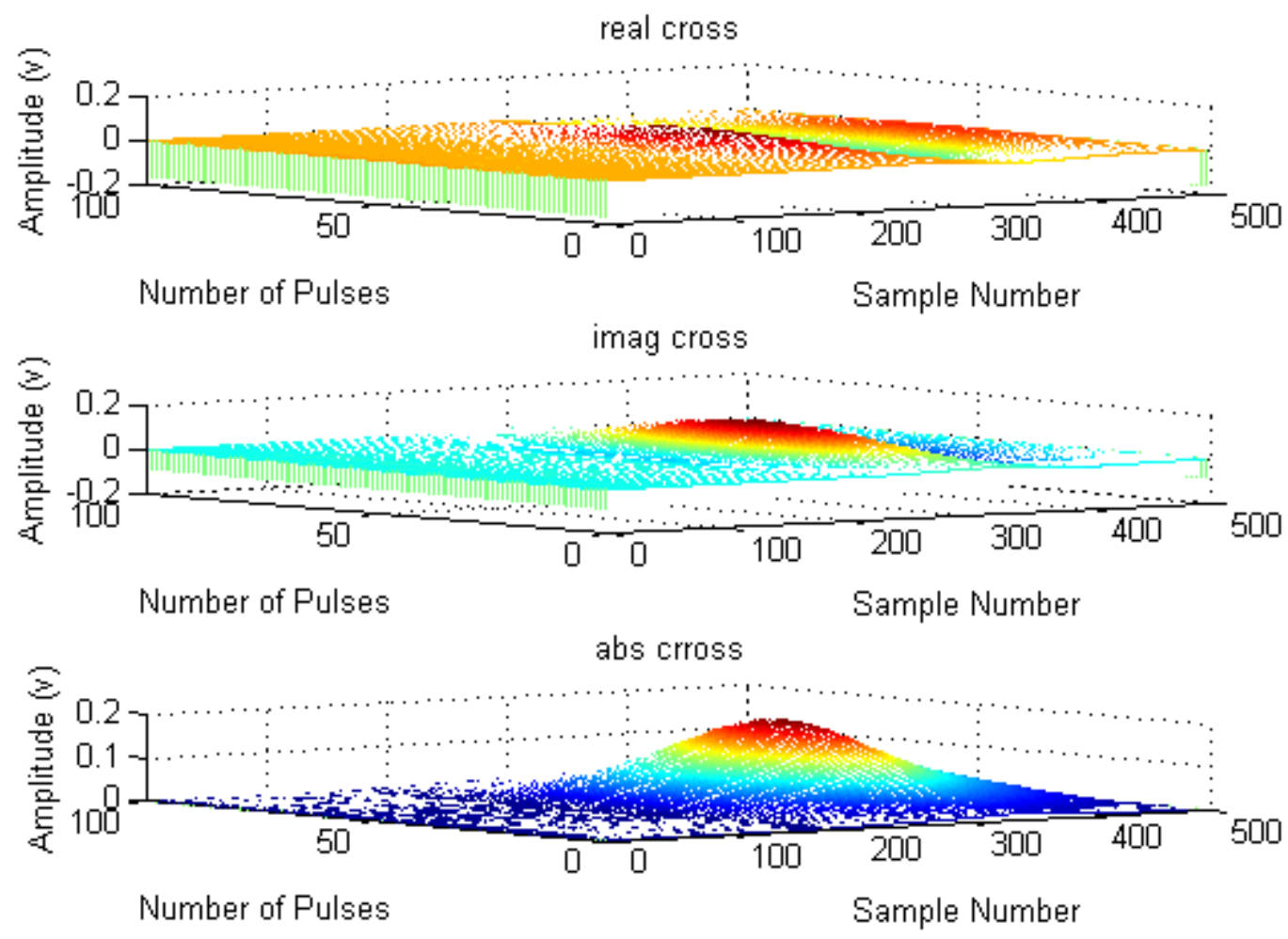

Figure 19: Cross-Correlate the TX and RX signals. 


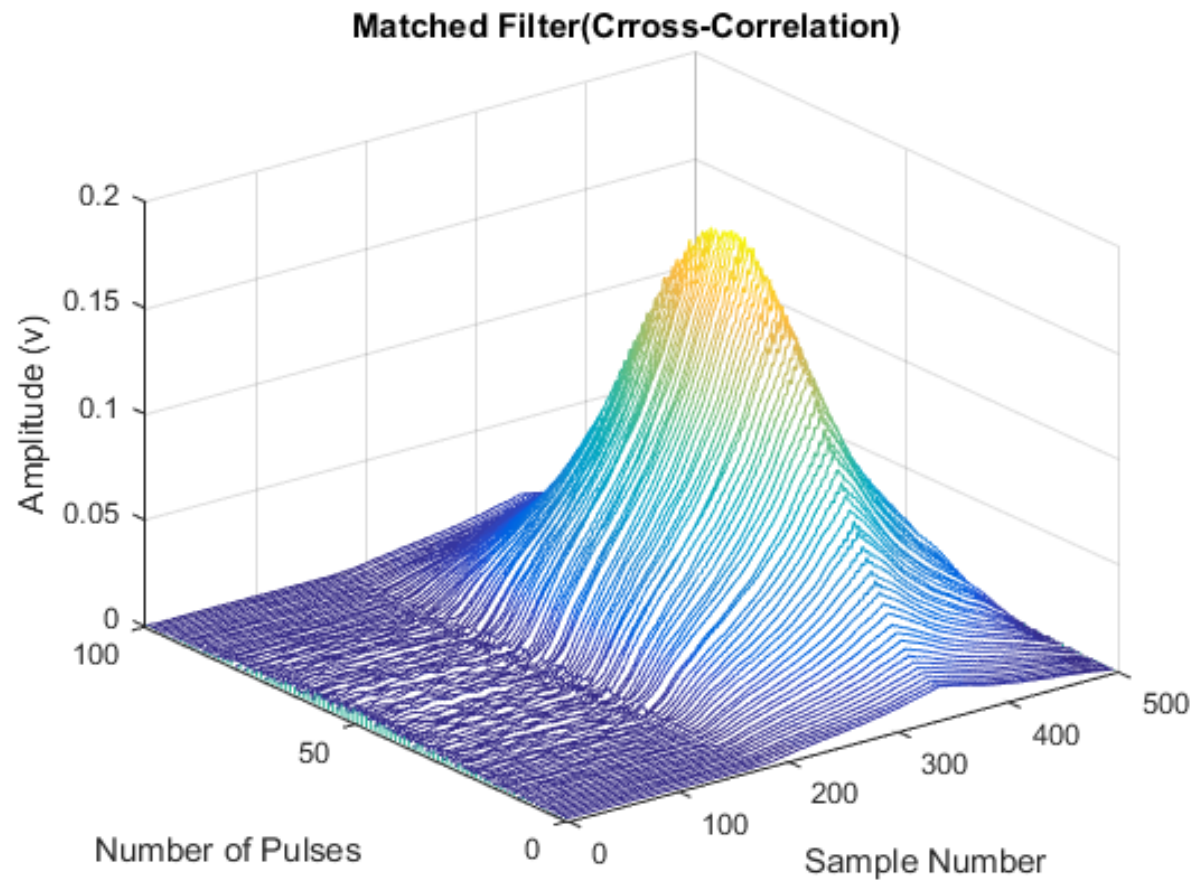

Figure 20: Absolute Cross-Correlate the TX and RX signals. 


\section{Fast Fourier Transform (FFT)}

FFT processing of the rows of the data above matrix results in Doppler decomposition as illustrated in Figure 21.

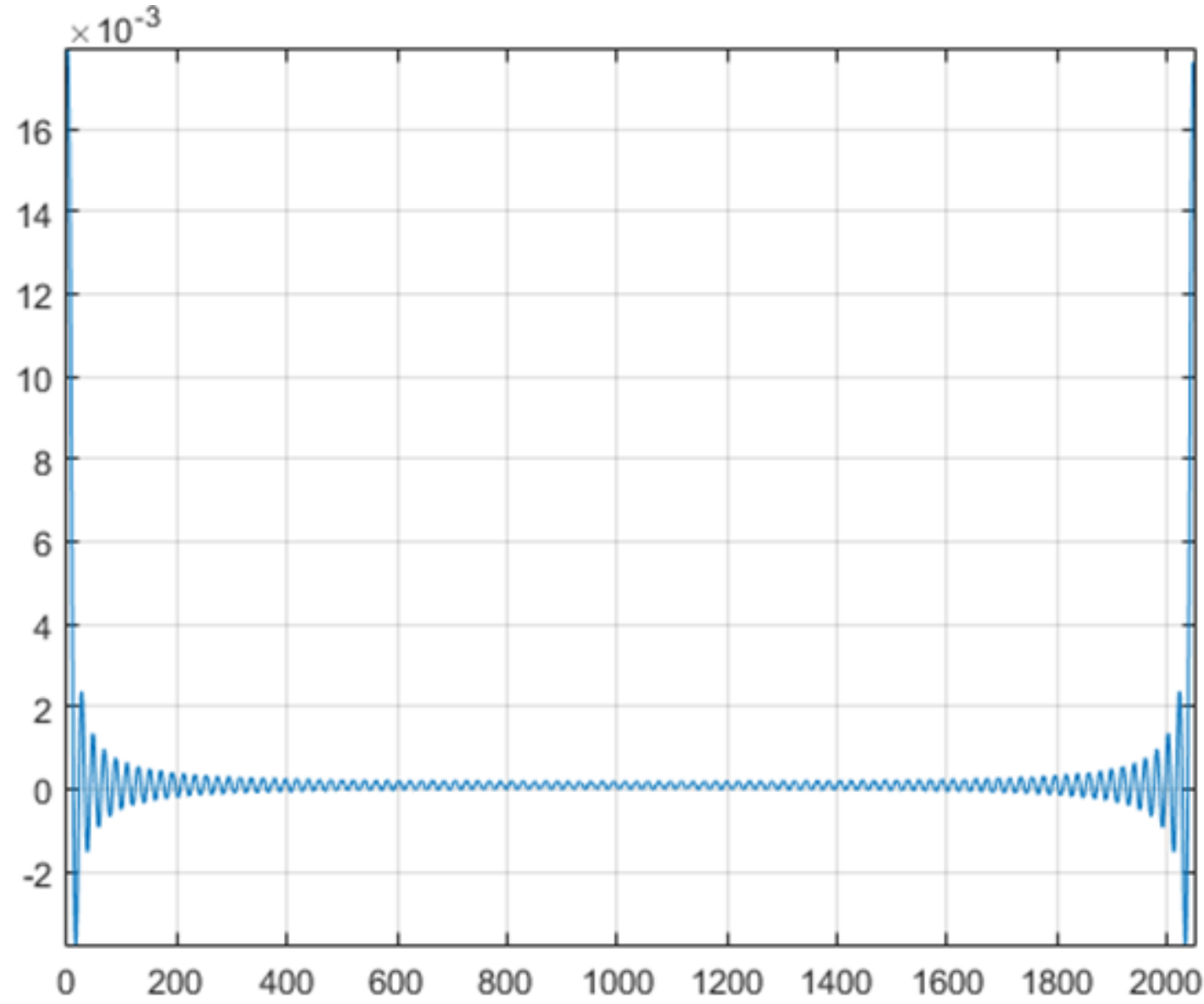

Figure 21: The result of the FFT process. 


\section{E. Doppler Frequency}

Figure 22 shows the Rang-Doppler image which is the proof of our invention.

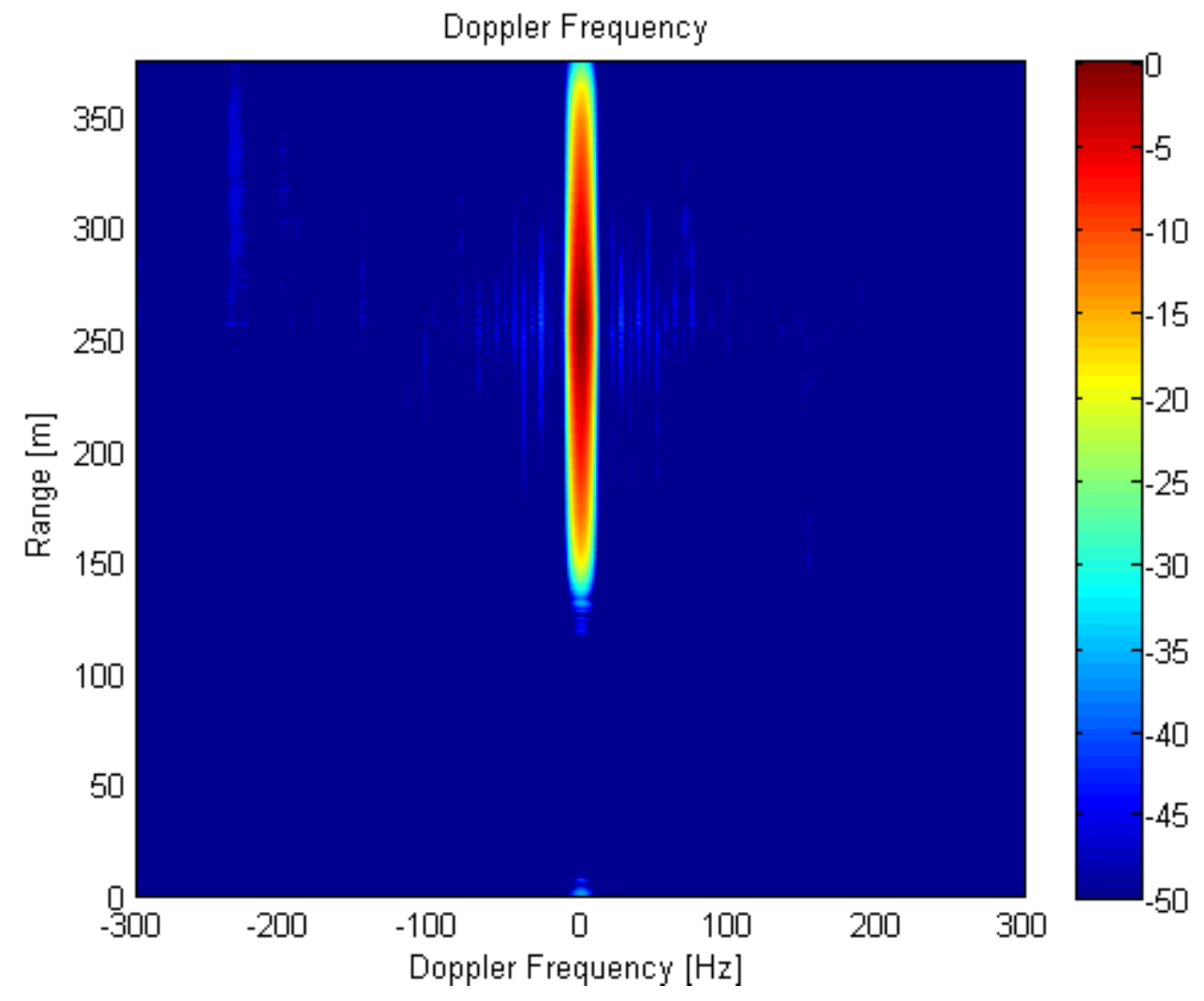

Figure 22: Range-Doppler image. 


\section{CHAPTER 5}

\section{THE INVENTION'S DESIGN}

\subsection{The Analog Part}

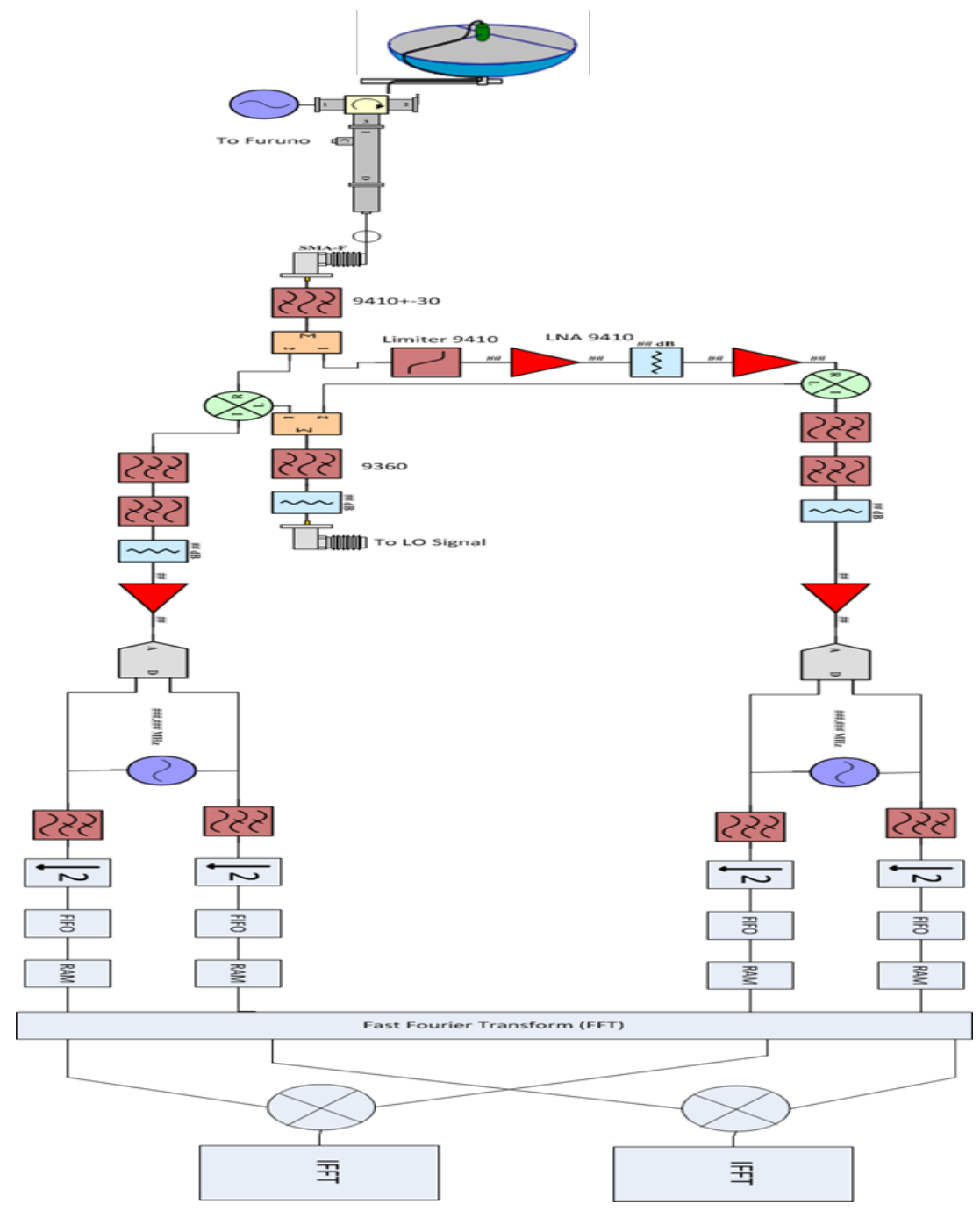

Figure 23: The analog chain components connected to the FPGA. 
Figure 23 shows the invention with the analog chain components. The circuit that we are analyzing is one of the main parts of coherent radar processing. This circuit is the first step of taking and attenuating the sample of the TX and RX signals. The non-coherent marine radar Furuno DRS25A operated the transmitted signal at $25 \mathrm{~kW}(75 \mathrm{dBm})$, which need to be attenuated to get analyzed and processed. The operation frequency of the marine radar is $9410 \mathrm{MHz}$, and the vector network analyzer measured it and confirmed by the oscillator. The sample of the transmitted signal was attenuated by $50 \mathrm{dBm}$ at the magnetron and then attenuated again by $20 \mathrm{dBm}$ before entering to the frequency mixer at (3400- $15000 \mathrm{MHz})$. The frequency mixer is connected to LO side with splitter (7.4 12.6 GHz) that split the clock signal that synchronized the transmitted signal and the received signal. The IF side of the frequency is connected to low pass filter (50 OHM DC- $96 \mathrm{MHz}$ ) then to DC-Block (50 OHM). The final destination of the transmitted sample is done by the FPGA. For received signal, the sample is entering a frequency mixer at (3400- $15000 \mathrm{MHz}$ ) after attenuated by $20 \mathrm{dBm}$ and the $\mathrm{LO}$ side of the mixer is connected to the clock signal for synchronization. A low-pass filter of (50 OHM DC- 140 $\mathrm{MHz}$ ) is connected to the IF side of the mixer. The received sample after filtering will enter to an ADC-Block before FPGA analysis. 


\subsection{The FPGA Part}

We configured our VHDL code in Xilinx Virtex 5 XC5VSX95T FPGA, which resides on the Sigmatec PX1500 board. Next are the main FPGA design steps, follow by the FPGA schematics and design resources and power consumption.

\subsubsection{FPGA design steps:}

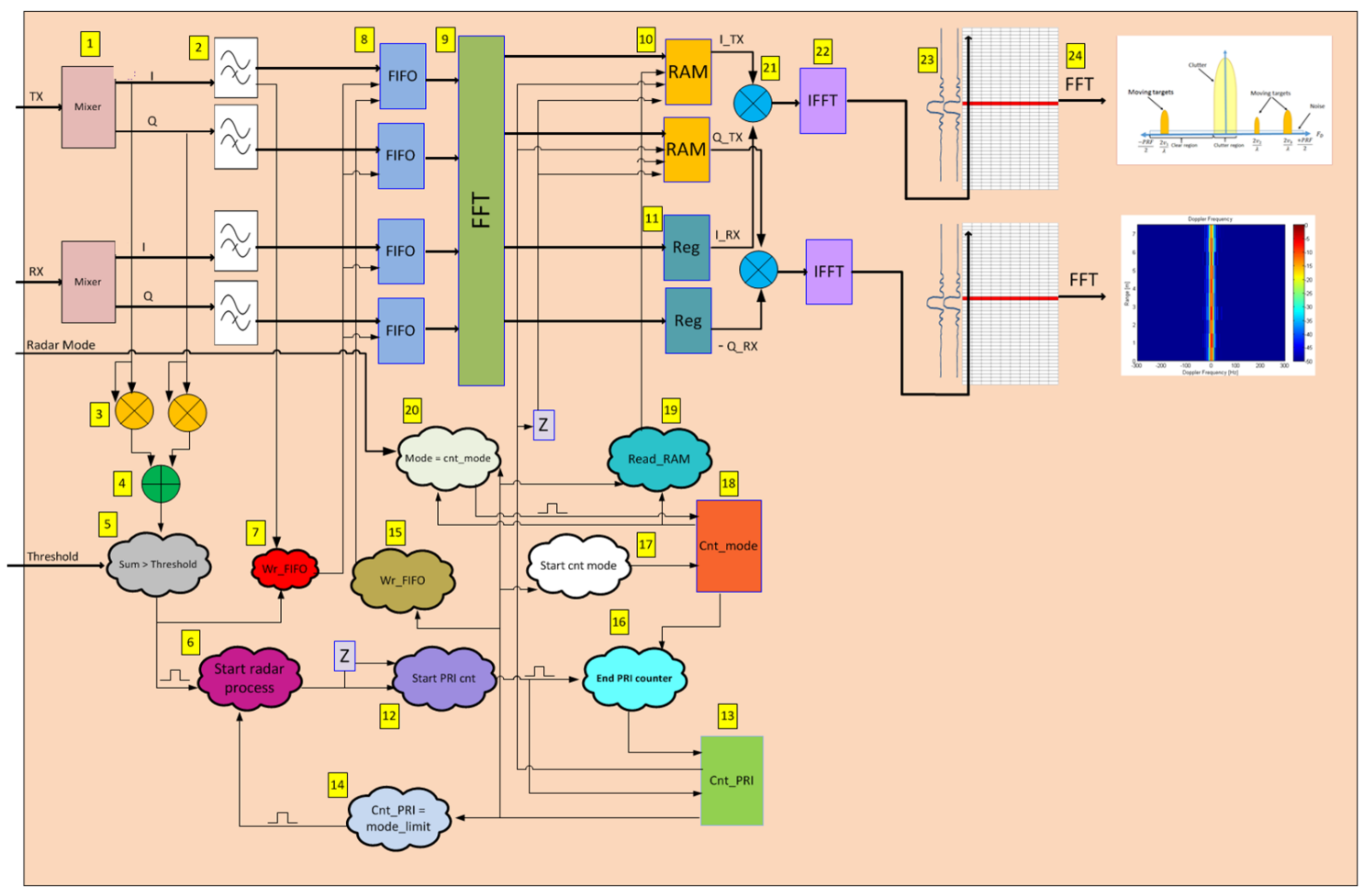

Figure 24: FPGA design and steps.

Figure 24 shows the FPGA design, which is broken down into the following steps.

1. The TX and RX signals are both RF signals. The outputs of the two mixers are centered on an IF of $50 \mathrm{MHz}$, and both signals are I-Q demodulated.

2. The outputs of the mixer are fed to $50 \mathrm{MHz}$ Lowpass filter and then decimated by 2. 
3. The TX I and Q signals are both squared to get the absolute value, so it will be compared to the threshold value to detect the new TX pulse.

4. Adding $\mathrm{I}^{2}$ and $\mathrm{Q}^{2}$ together, so they can be compared to the threshold.

5. This process compares the sum of $\mathrm{I}^{2}$ and $\mathrm{Q}^{2}$ to the given threshold.

6. If the sum is equal or greater than the threshold, a pulse will be created to start the processing the incoming data.

7. Once the TX pulse is detected, the enable write FIFO signal will be active to write incoming data to four FIFOs (two for TX, and two for RX)

8. Since the system clock is $200 \mathrm{MHZ}$ and the data got decimated by 2 to $100 \mathrm{MHz}$, we need a FIFO to store data then read it out of the FIFO at $200 \mathrm{MHz}$.

9. FFT all the filtered data.

10. Since the maximum Xilinx Virtex 5 FFT bins is 65536 and the maximum PRI length is 363636 , so we have to save the TX FFT data in a RAM, and then read it multiple times to make sure that we cross-correlated the whole RX signal. The number of reading the data out of the RAM depends on the radar modes; see Figure 25 for the radar mode operation.

11. Since we have to save the TX data in a RAM for some time before we read it out, we have to save the RX signal in the registers to make sure the two data.

12. When the TX pulse got detected, the system creates a one clock cycle pulse to get the PRI counter starts. 
13. The PRI counter counts the length's numbers of the PRI period. Again the period depends on the radar mode of operation.

14. When the counter reaches the maximum PRI length, the system creates a pulse to indicate the end of the match filter processing.

15. The PRI counter also controls the read of the FIFOs. When the PRI counter reaches a certain value, the FIFO starts reading out the stored data.

16. The length of the PRI counter depends on the radar mode operation. So when the counter mode reaches the operation mode value, the system creates a pulse to reset the PRI counter and wait for a new TX pulse.

17. When the PRI counter starts, it also sends a pulse to the mode counter to starts count.

18. The radar has five mode operations $(3000 \mathrm{~Hz}, 1400 \mathrm{~Hz}, 1000 \mathrm{~Hz}, 600 \mathrm{~Hz}$, and $500 \mathrm{~Hz}$ ). The mode counter receives the operation mode from the user and then controls the operation of the system according to the user input mode.

19. The mode counter tells the system how many times to read the data out of the RAM for the match filter processing.

20. When the mode counter reaches the number corresponding to the desired mode, the system sends a pulse to reset the mode counter and wait for a new TX pulse.

21. The TX data out of the RAMs get multiplied by the conjugate RX data of the registers. 
22. The data out of the complex multiplier gets IFFT process to produce the result of the matched filter.

23. The output data from the IFFT block gets arranged in 2 D matrixes. Each column contains the range (the fast time), and the row contains the pulse number (the slow time.)

24. Finally, each row of the matrix gets FFT process to produce the Doppler frequencies.

The data from the FFT channel was processed in MATLAB technique and the resulting range profiles analyzed to find the velocity of the targets.

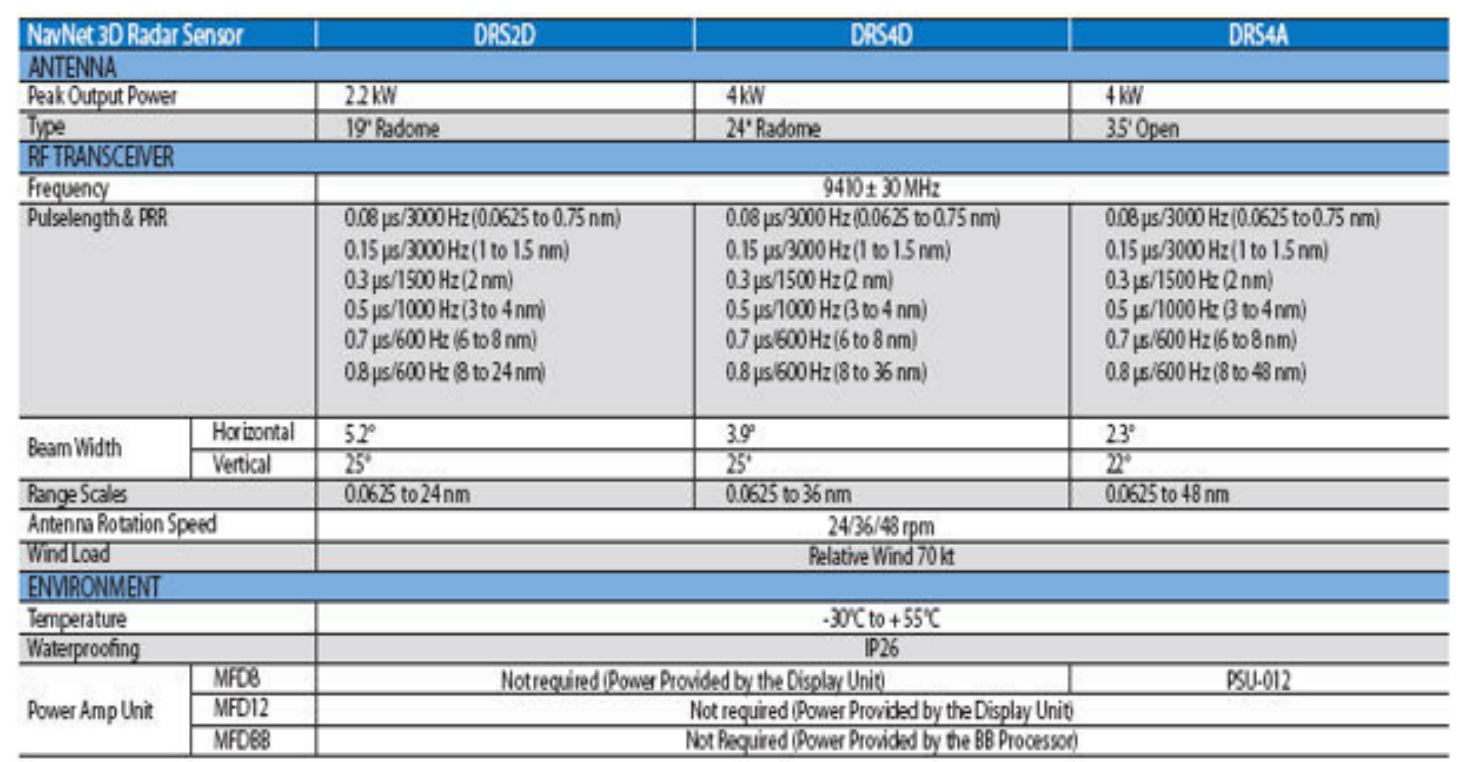

Figure 25: Marine Radar Specifications. 


\subsection{The Resources and Schematics:}

This section shows Xilinx ISE design summary and schematics.

Figure 26, Figure 27, and Figure 28 show the FPGA synthesis report and design utility summary of the whole design.

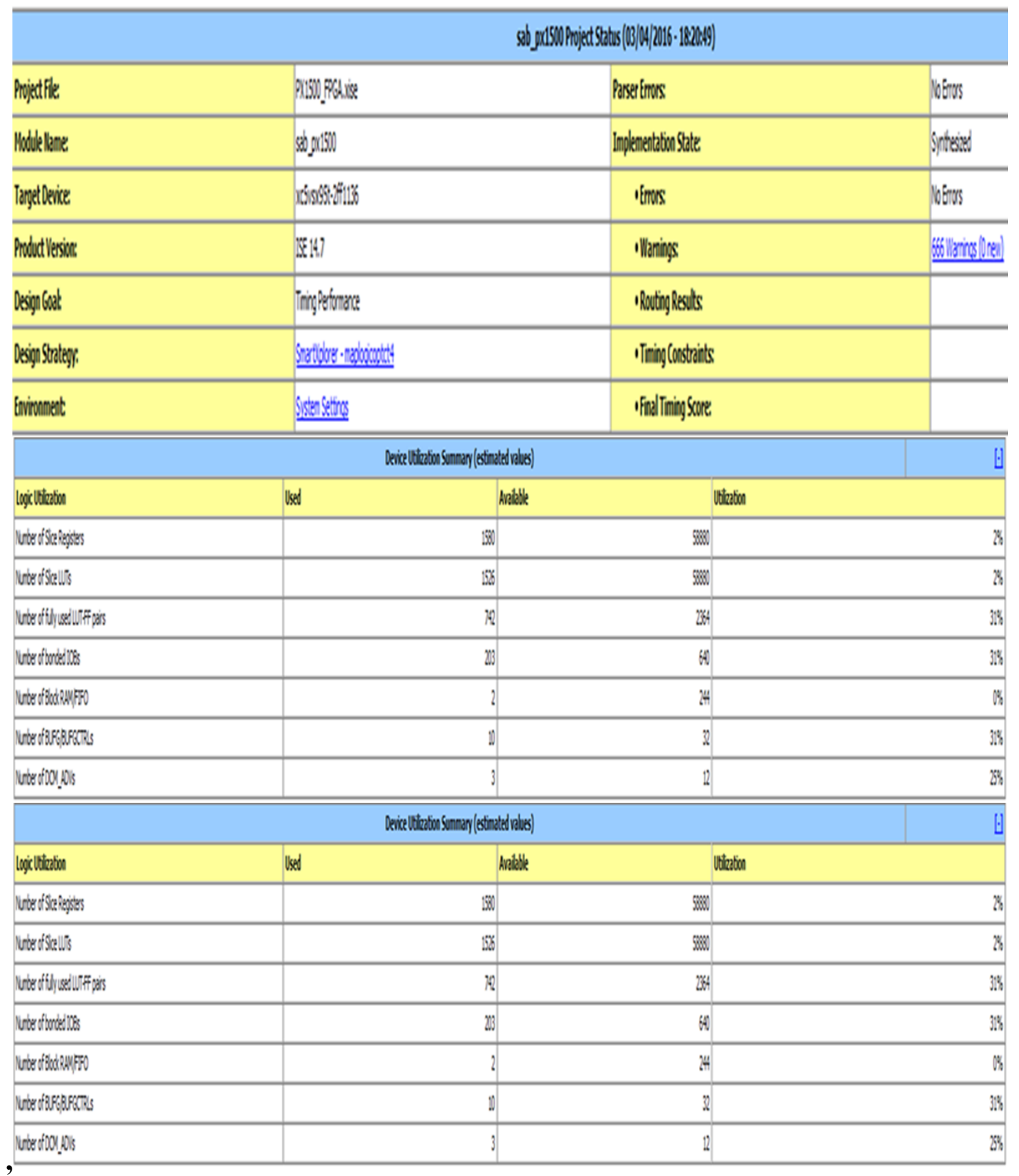

Figure 26: FPGA synthesis report. 


\begin{tabular}{|c|c|c|c|c|}
\hline \multicolumn{5}{|c|}{ Device Uttilization Summary } \\
\hline Slice Logic Utilization & Used & Avalibble & Utirzation & Mote(5) \\
\hline Number of filce Registeris & 10,22 & 58800 & $17 \%$ & \\
\hline Whber used as Flip Fipss & 10,212 & & & \\
\hline Nurber of fice L/S & 6,212 & 58800 & $10 \%$ & \\
\hline Nunber used a a logic & 2,177 & 59800 & $3 \%$ & 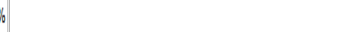 \\
\hline Nurmer using O6 oupuptony & 1,633 & & & \\
\hline Nurner sisro 05 oulpitony & 293 & & & \\
\hline Nurter wisho 05 and ch & 251 & & & \\
\hline Wrnber used bs Menoroy & 4,218 & 24,20 & $17 \%$ & \\
\hline 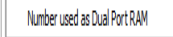 & 4,44 & & & \\
\hline Nunber sing C6atut ony & 4,104 & & & \\
\hline Nurber sing C5 atatotony & 20) & & & \\
\hline Nurbe : sing 05 ad 06 & 22 & & & \\
\hline Nurber used as ShiftRegigter & 74 & & & \\
\hline Nurber sing C6 atututony & 72 & & & \\
\hline Nurber singo5 atatutony & 2 & ? & & \\
\hline Winbo used ds exdadive route-tru & 25 & & & \\
\hline Nurber of foule:trins & 321 & & & \\
\hline Nonbs usirg C6 ouputady & 318 & & & \\
\hline Nrnbe usirg 05 oututionly & 3 & 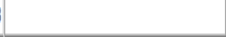 & & \\
\hline Nunber of occapided Sices & 3,750 & 14,720 & $25 \%$ & \\
\hline Nurber of LUT Fip Flep pairs Lsed & 11,048 & & & \\
\hline Nunber wht an unsed flip Flop & 928 & 11,049 & $8 \%$ & $/$ \\
\hline Wraber wh a nusced UT & 4,628 & 11,049 & $41 \%$ & \\
\hline Nenbe of fuly ysed UTFF pairs & 5,493 & 11,049 & $49 \%$ & $\%$ \\
\hline Nanbe of urige conto sets & 729 & & & \\
\hline 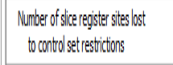 & 797 & 58800 & $1 \%$ & $/ 4$ \\
\hline Nurber of borced 10Bss & 269 & 640 & $4 \%$ & \\
\hline Minbe of LOCes:CCEs & 269 & 269 & $100 \%$ & \\
\hline 108 Fip Flops & 456 & & & \\
\hline Nurber of BodochANFFO & 28 & 24 & $11 \%$ & \\
\hline Nrinbe usirg Bod:RAM ony & 28 & & & \\
\hline Numer of $36 k$ EloxxeM/ ussd & 27 & & & \\
\hline 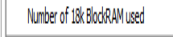 & 1 & . & & \\
\hline Total / Menryyused (BB] & 990 & 8,784 & $11 \%$ & \\
\hline Nunber of B FFG BVFGCTRLs & 5 & 32 & $15 \%$ & $/ /$ \\
\hline Nunbe used as EUFGs & 5 & 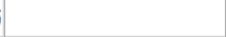 & & \\
\hline Number of DEAACTRLS.S & 6 & 2 & $27 \%$ & \\
\hline 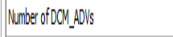 & 1 & .12 & $8 \%$ & $/ 4$ \\
\hline Nunber of D:P 49ES & 130 & 640 & $20 \%$ & \\
\hline 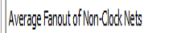 & 242 & & & \\
\hline
\end{tabular}

Figure 27: Device Utility Summary. 


\section{Report utilization}

Estimated resources are compared with xc5vsx 95 tff $1136-2$.

Show More Details

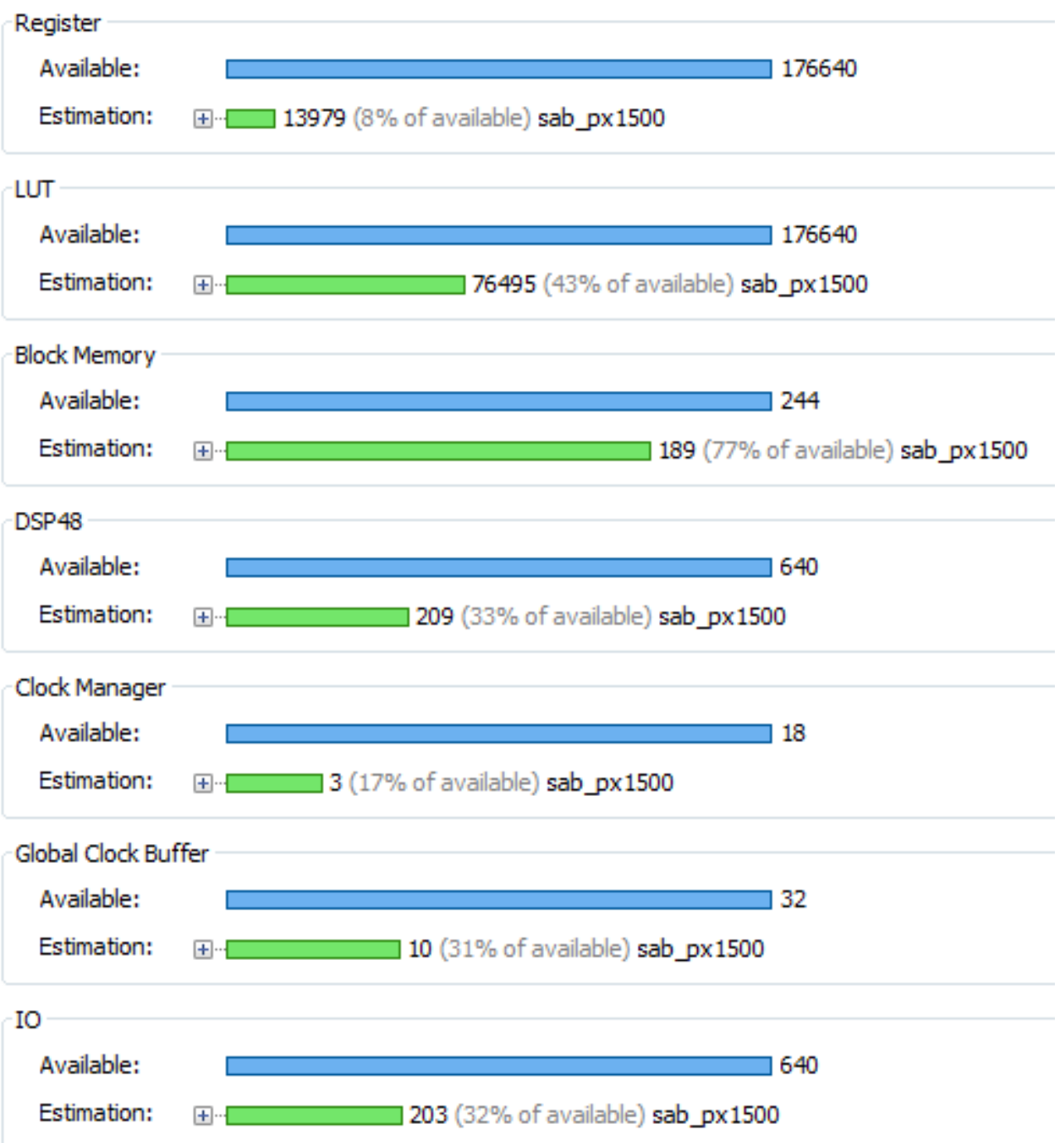

Figure 28: Estimated resources. 
Figure 29 shows the inputs and the outputs signal of the FPGA marine radar design. The user can choose the radar mode as well as setting the required threshold.

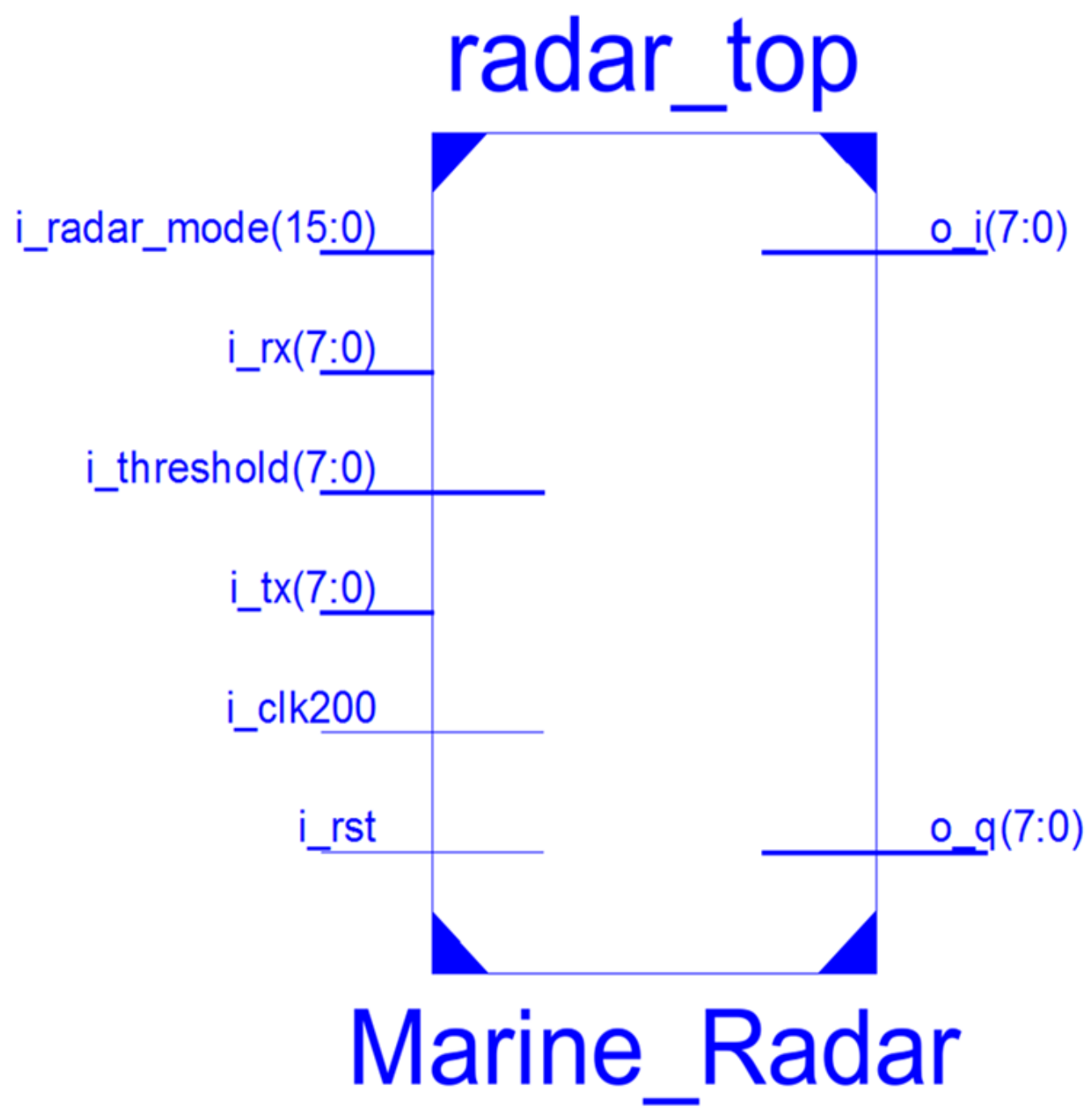

Figure 29: Radar I/O Pins. 
Figure 30 shows the RTL for the entire FPGA design.

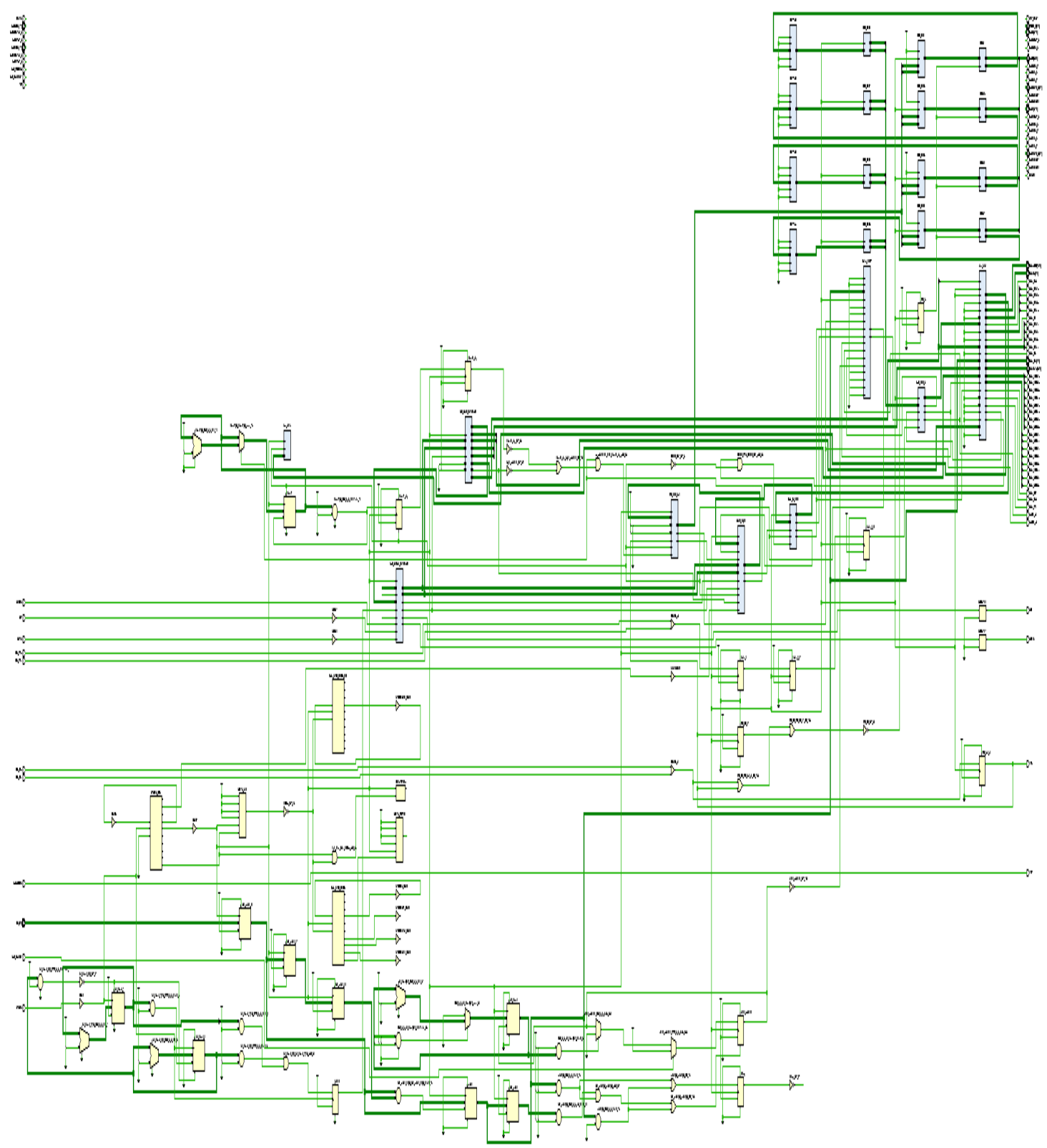

Figure 30: FPGA Board RTL. 
Figure 31 shows the RTL for the radar design.

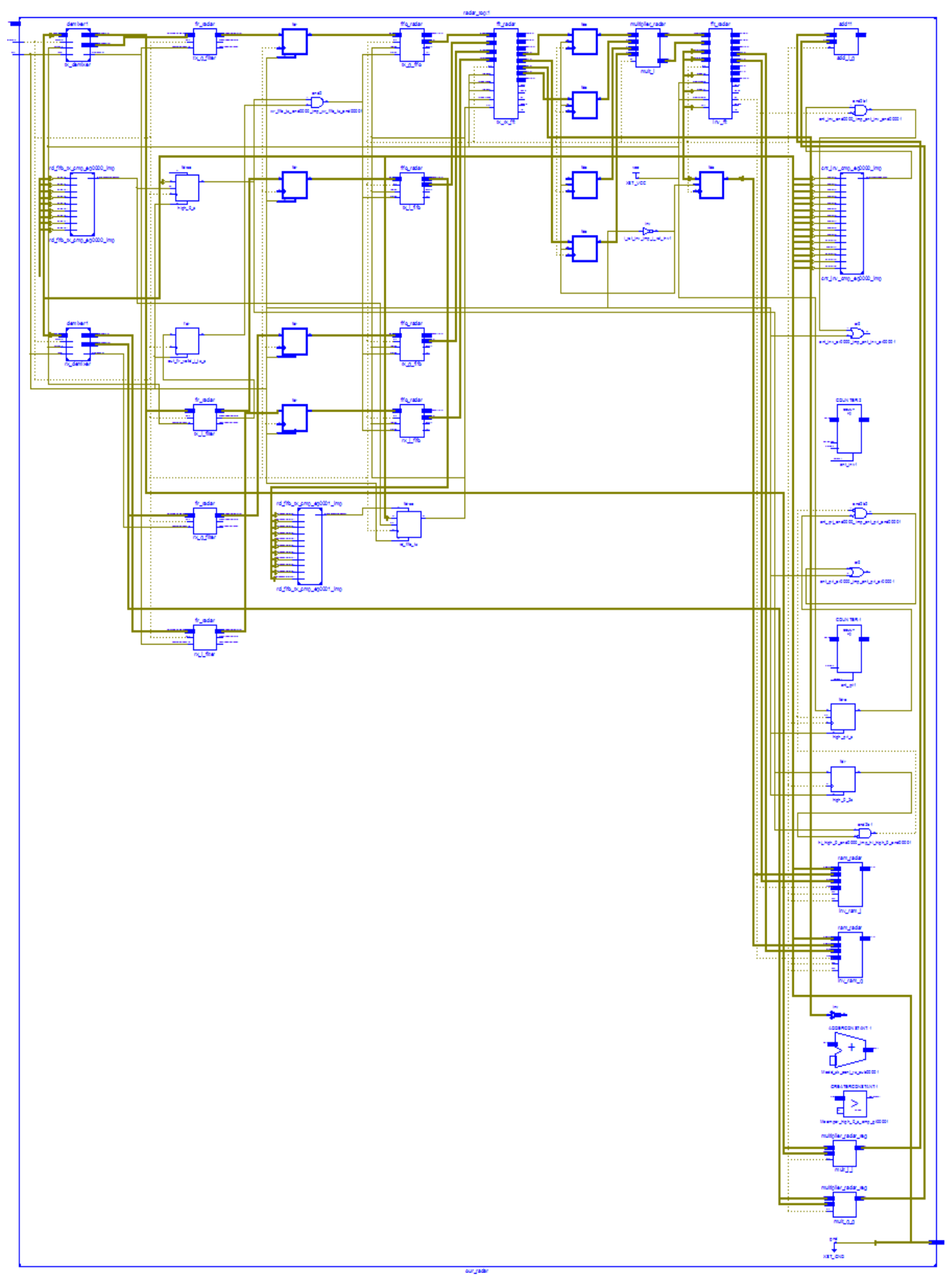

Figure 31: Radar Design RTL. 
Figure 32 shows the FPGA marine radar design blocks and the connections between them.

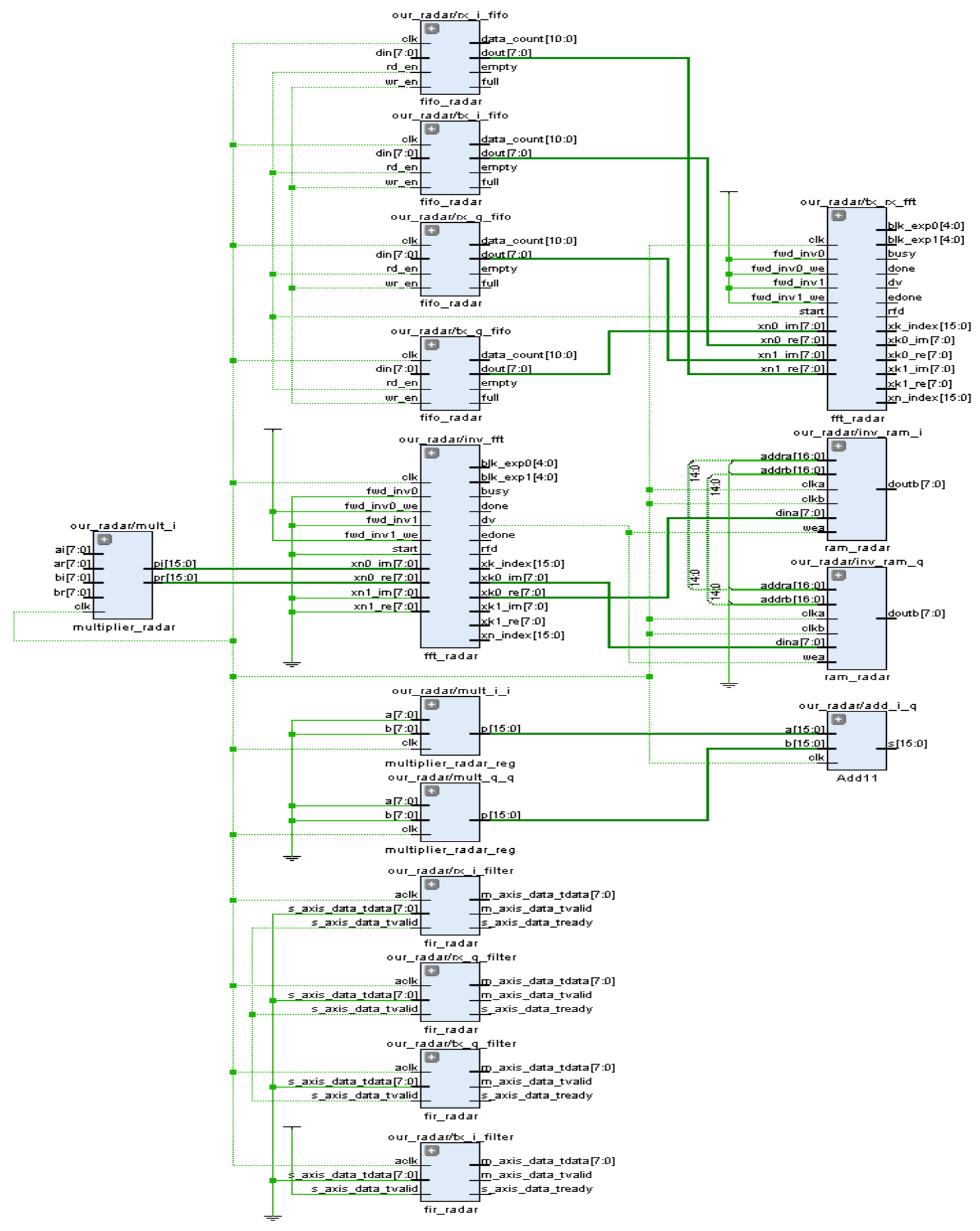

Figure 32: Main Radar Blocks. 
Figure 33 shows the marine radar FPGA gates and registers.

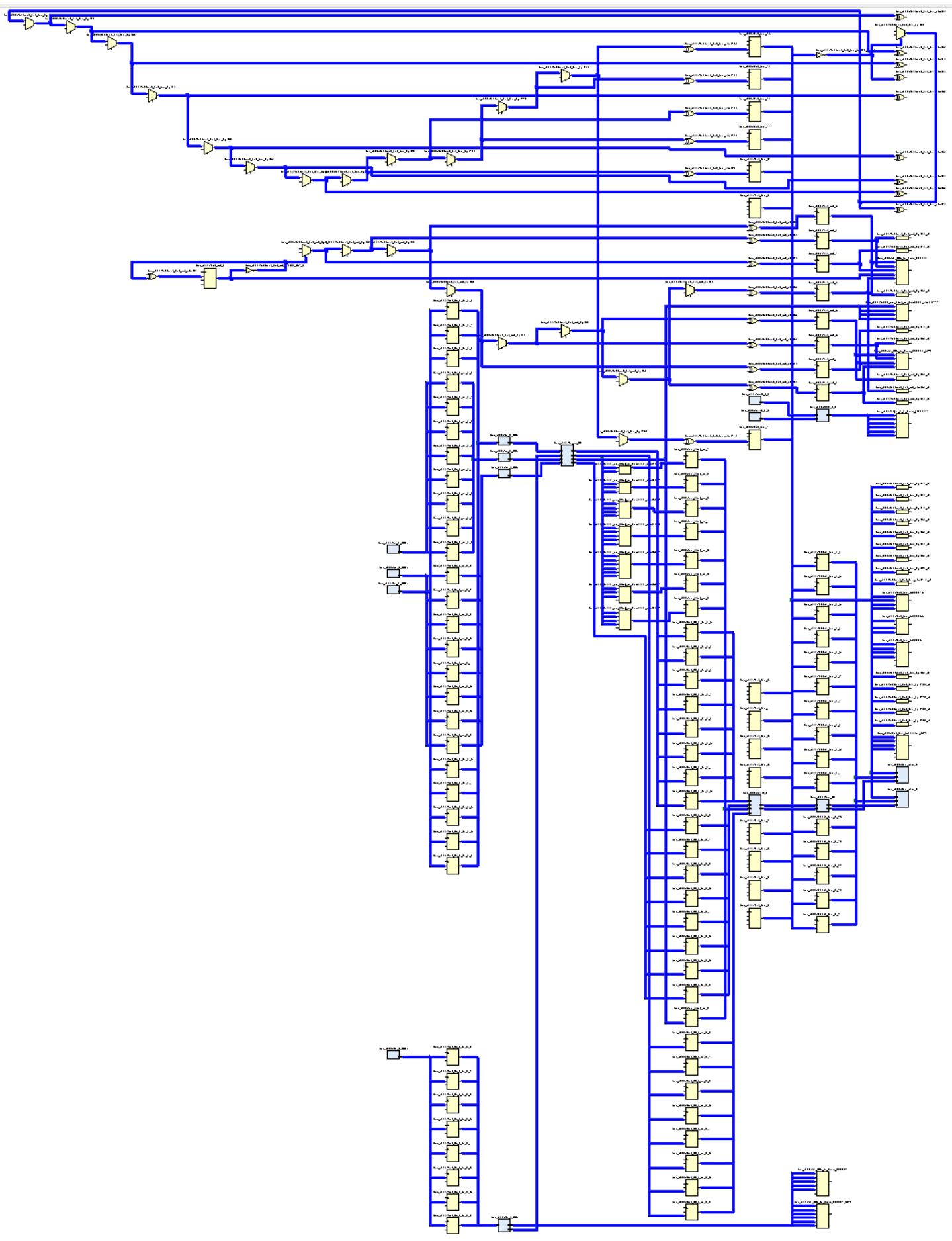

Figure 33: Radar Registers. 
Figure 34and Figure 35 show the FPGA density and hierarchy respectively.

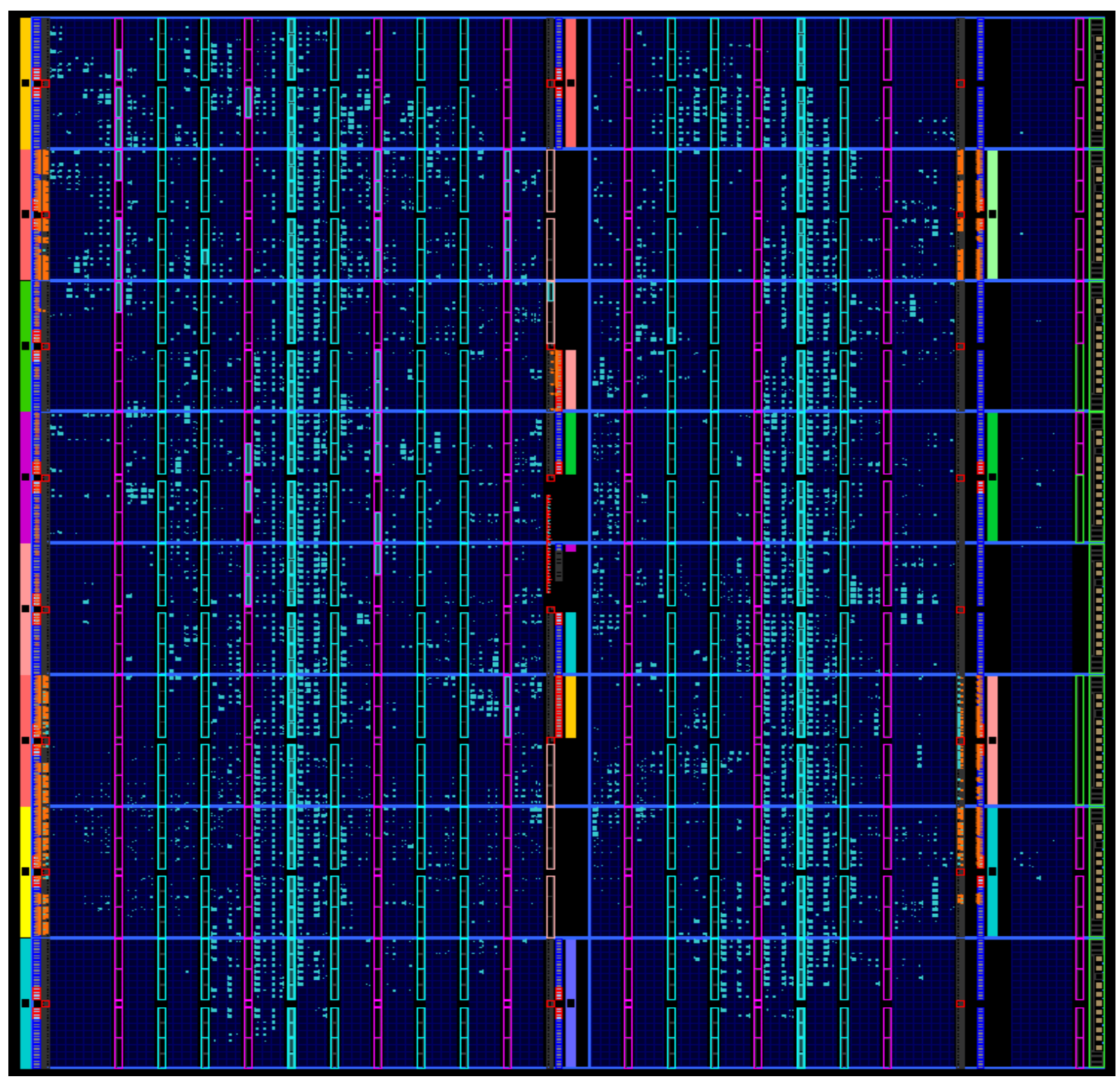

Figure 34: FPGA Density. 


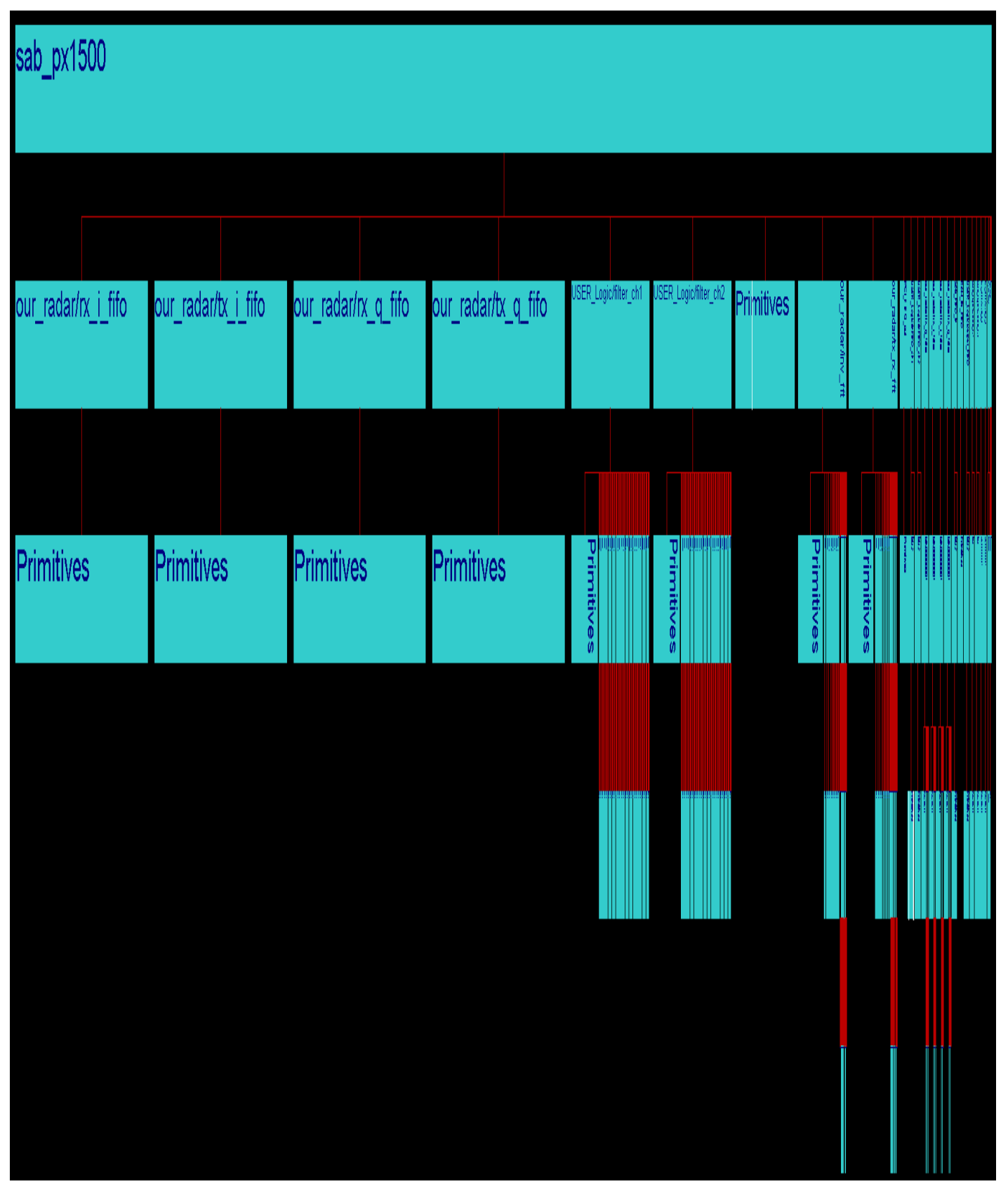

Figure 35: FPGA Hierarchy. 
Figure 36 shows the whole design power consumption.

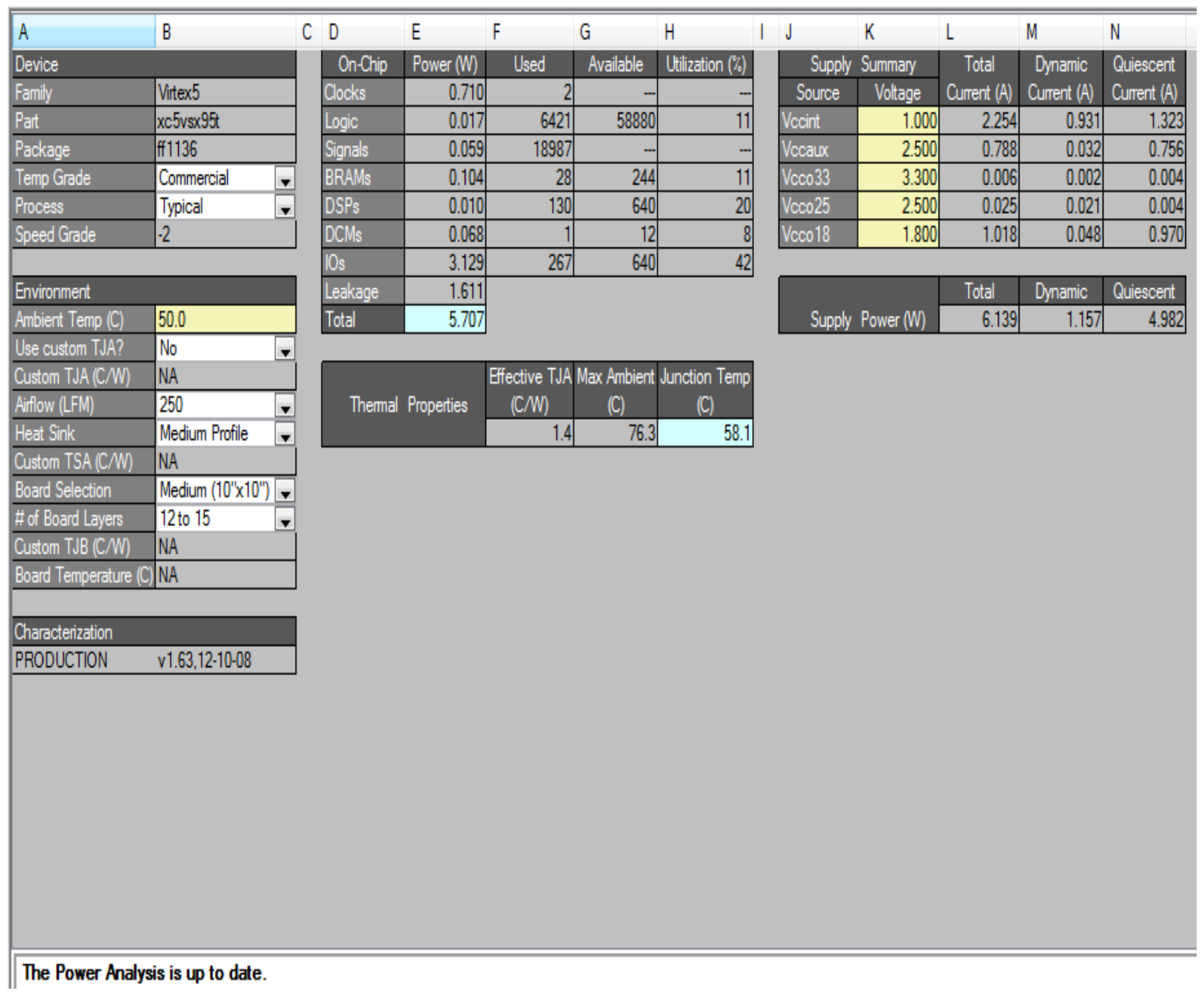

Figure 36: Radar FPGA Power Consumption. 


\section{CHAPTER 6}

\section{FINDING AND RESULTS}

\subsection{Findings}

In this section, one TX and one RX PRI signal will be tracked from it entering the FPGA block, processing through the entire design, until leaving the FPGA. The result of the Doppler Frequencies is also shown at the end of this section.

Figure 37 and Figure 38 show the TX and RX signals when entering the radar FPGA top level.

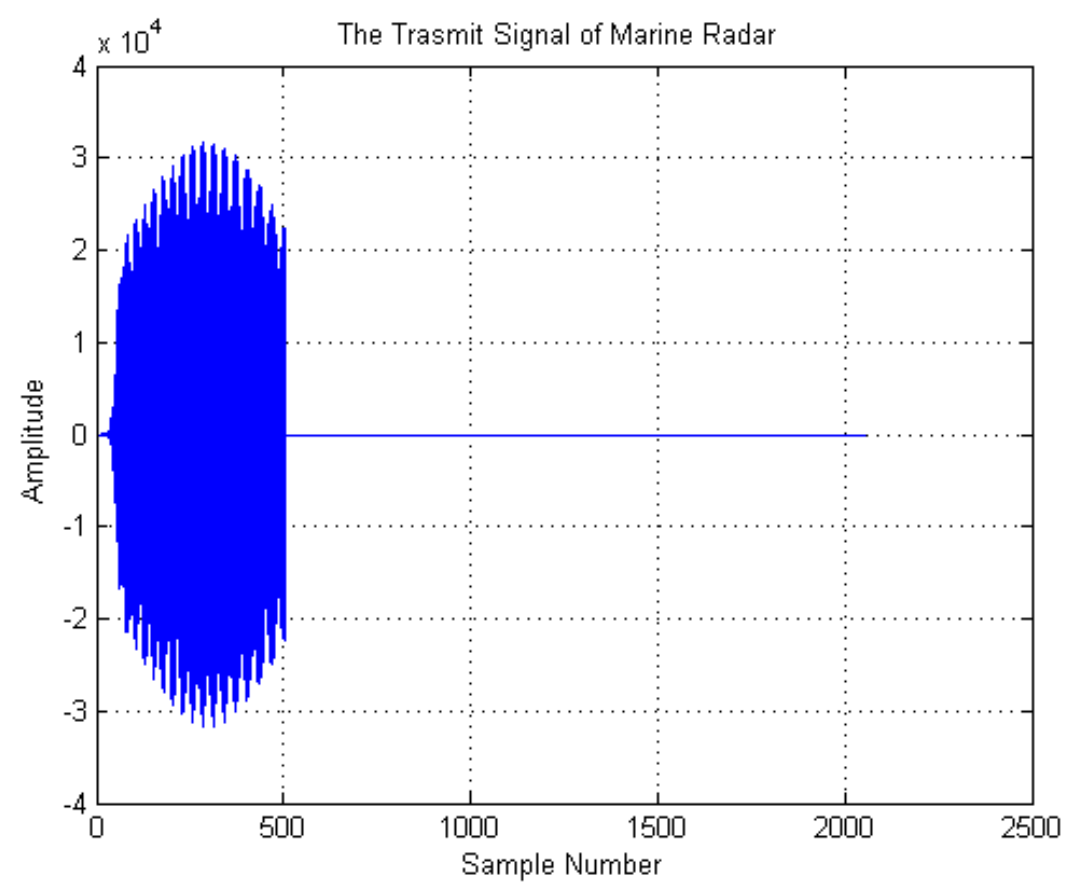

Figure 37: One TX pulse. 


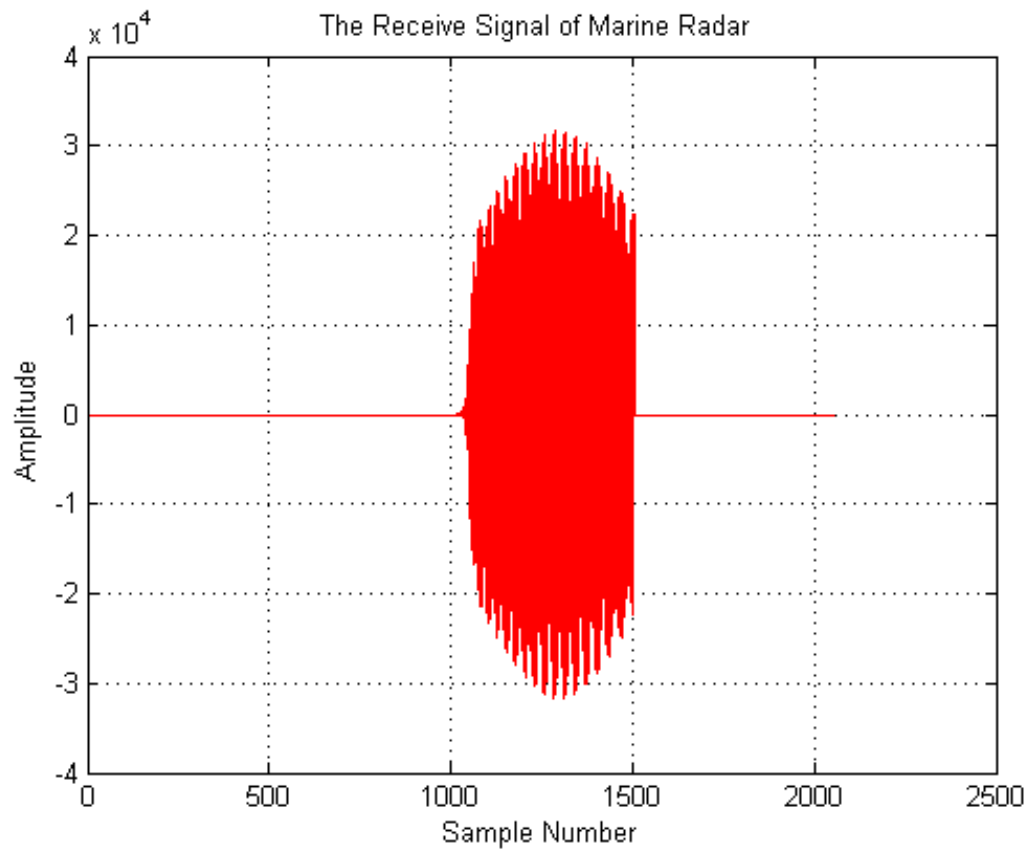

Figure 38: One RX pulse. 
Both TX and RX signals are get mixed with $50 \mathrm{MHz}$ LO, to go from RF to IF signal.

Figure 39 and Figure 40 show an example of TX signal after it goes through the mixer with its I and Q signals in the same plot, then the same signals in its plots.

Also Figure 41, Figure 42, Figure 43, and Figure 44 show I Tx, I RX, Q TX, and Q RX respectively.

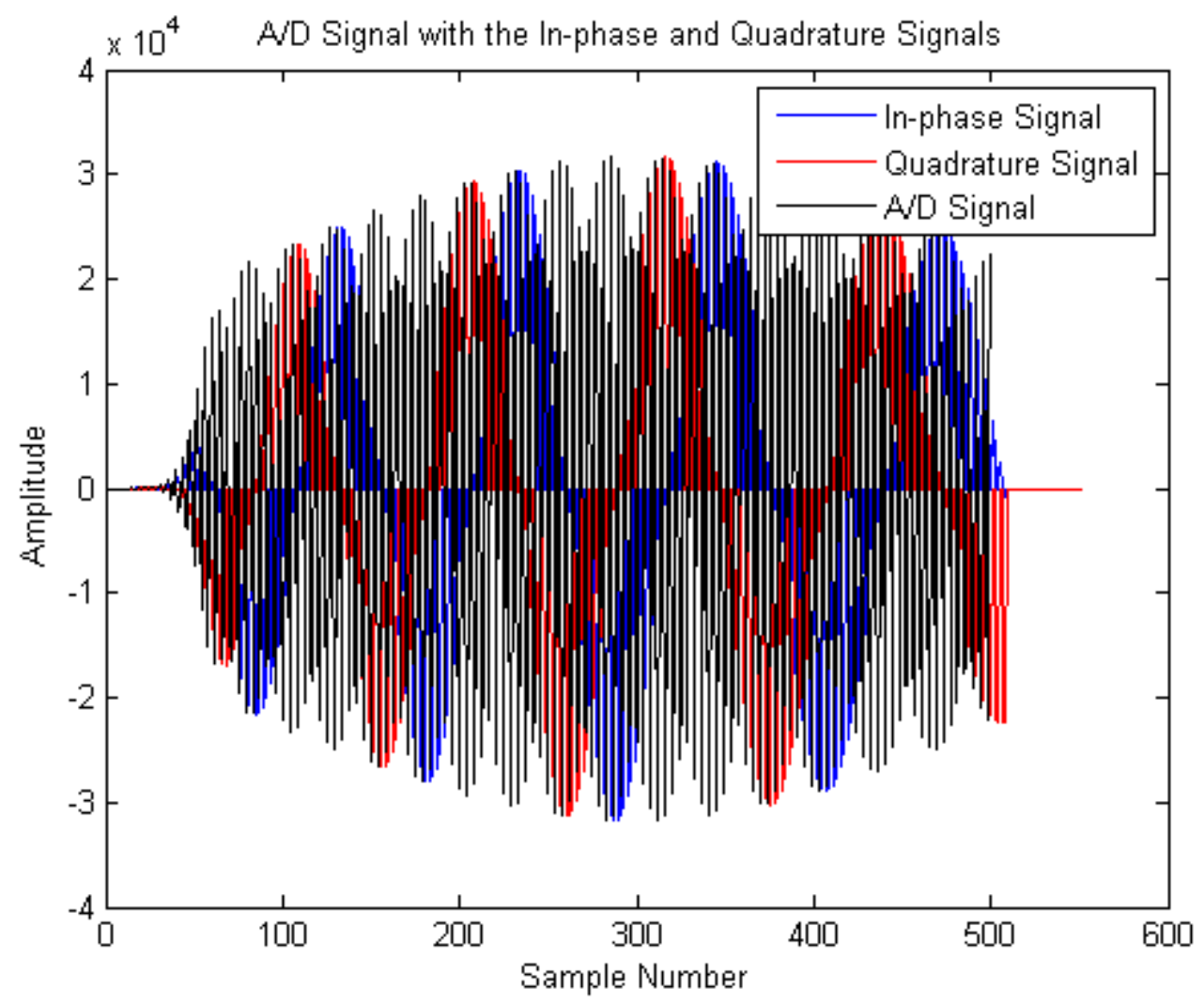

Figure 39: TX with I and $Q$ signal on one plot. 

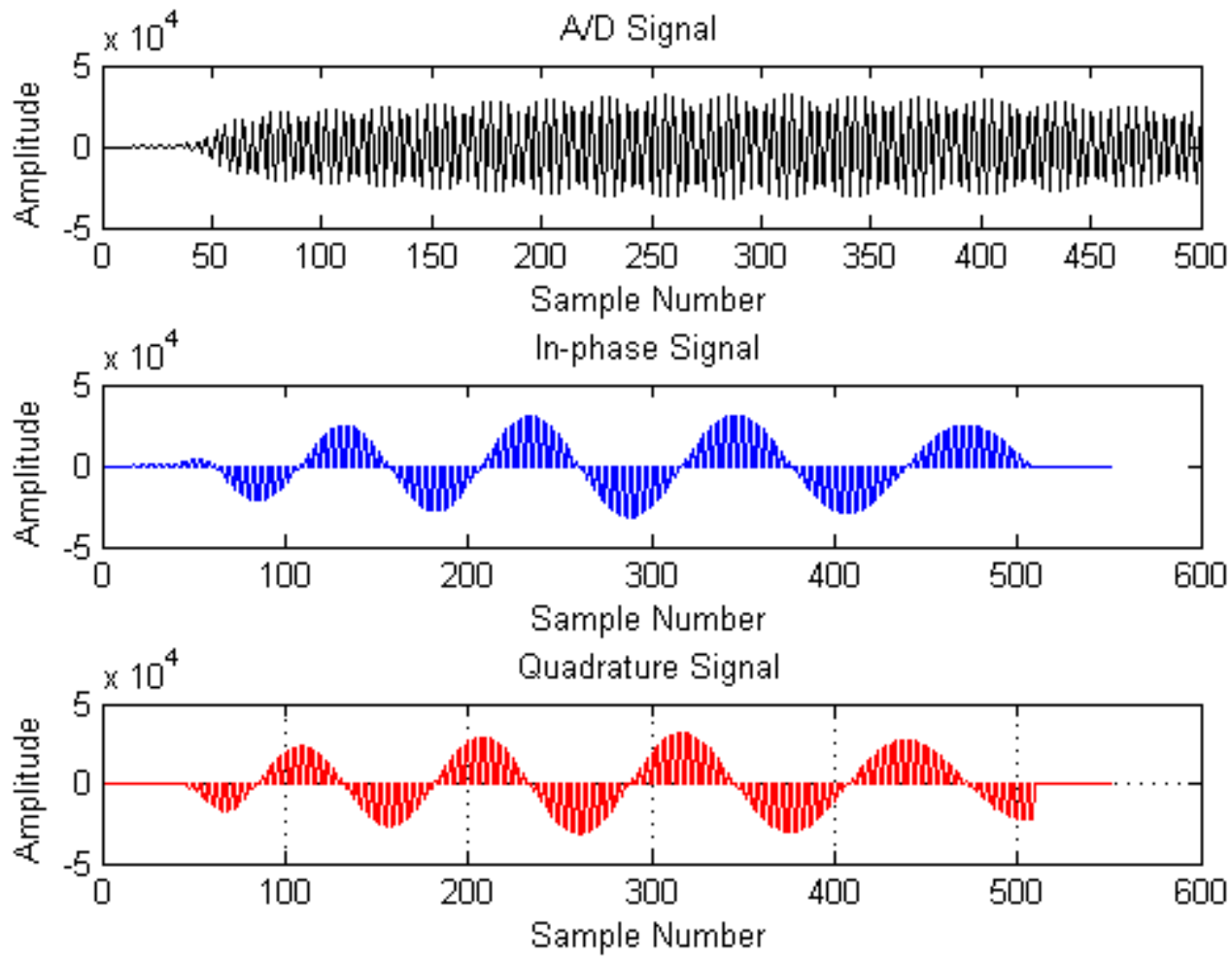

Figure 40: TX with I and $Q$ signal on separate plots 


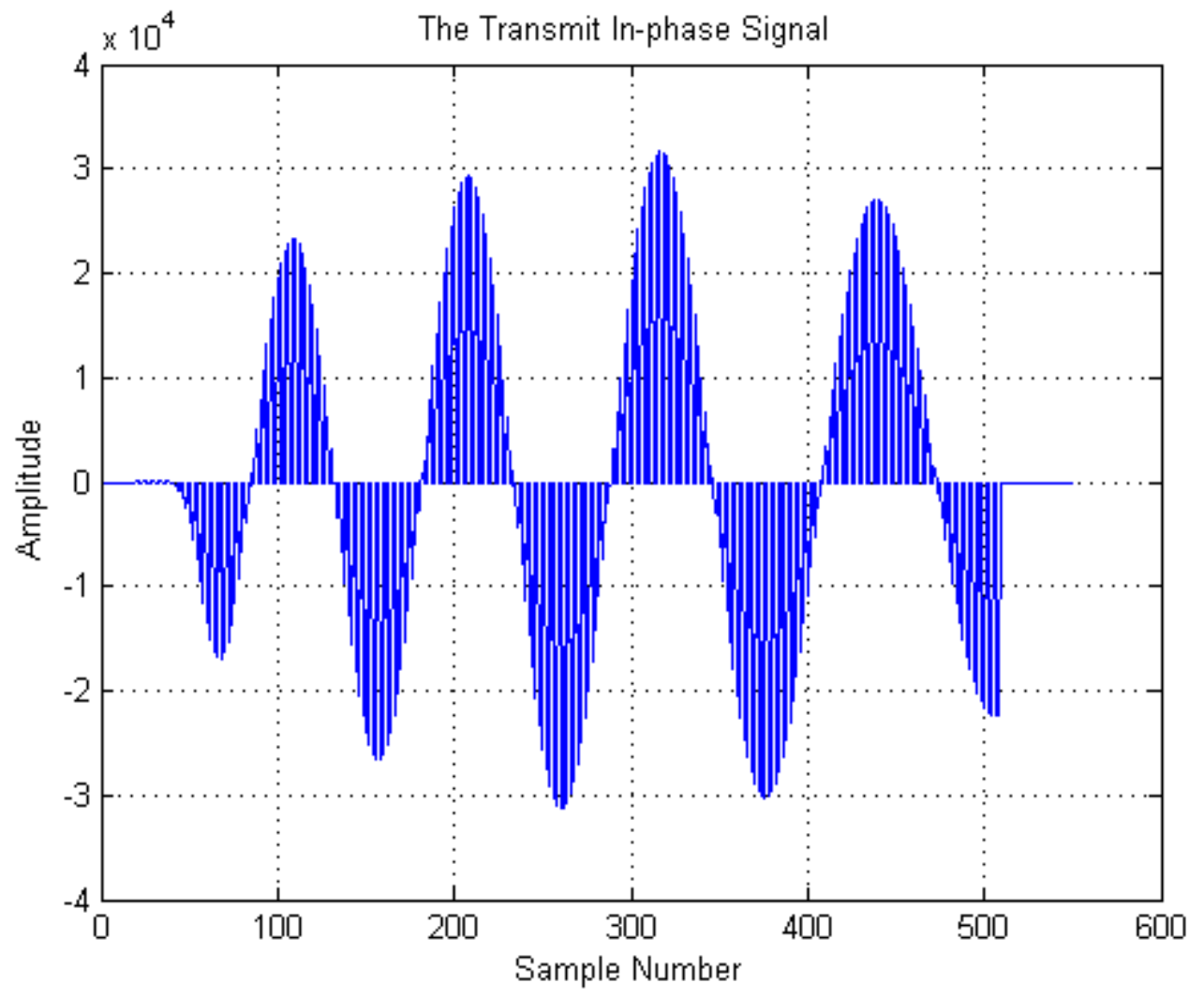

Figure 41: I TX Signal. 


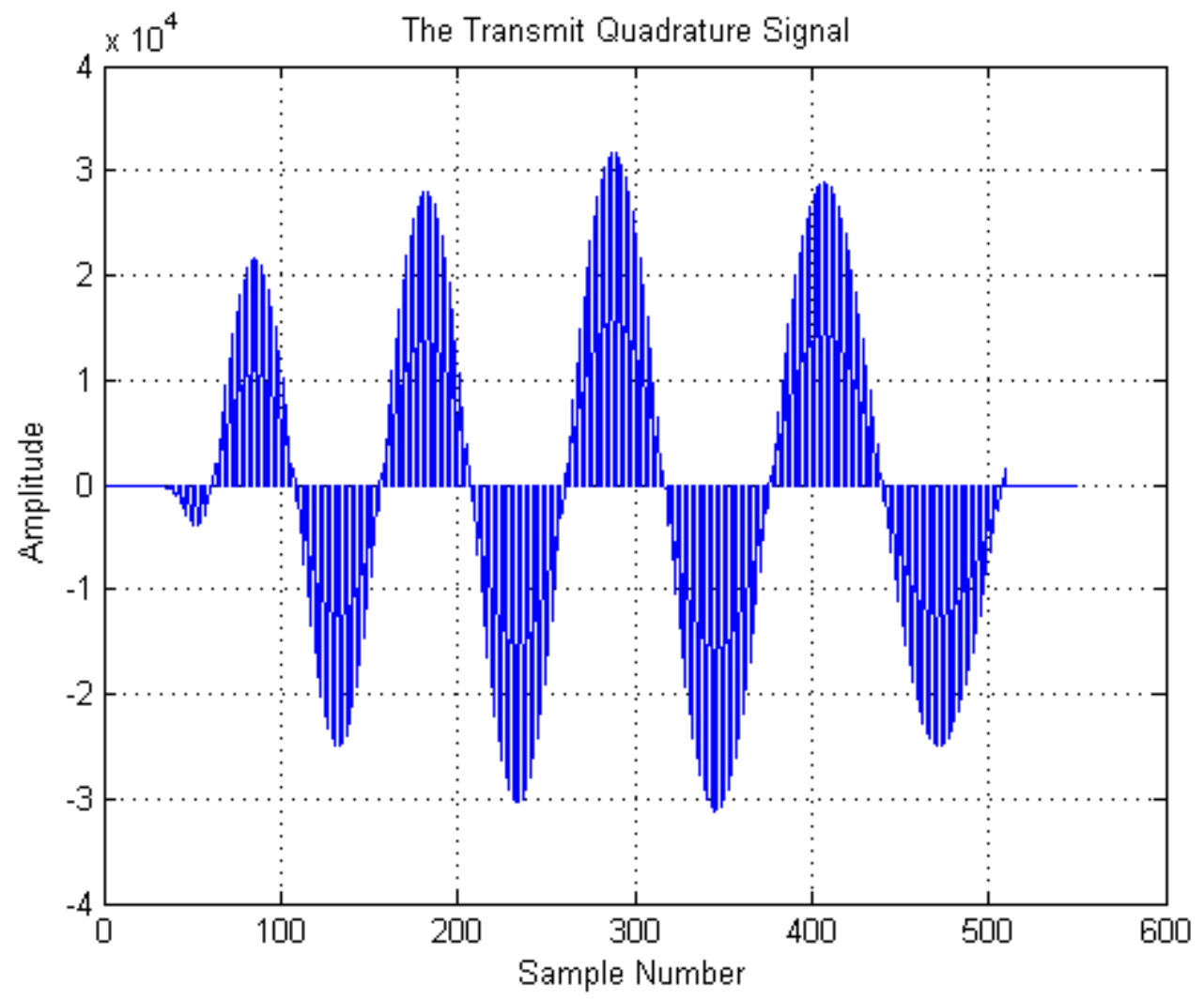

Figure 42: Q TX Signal. 


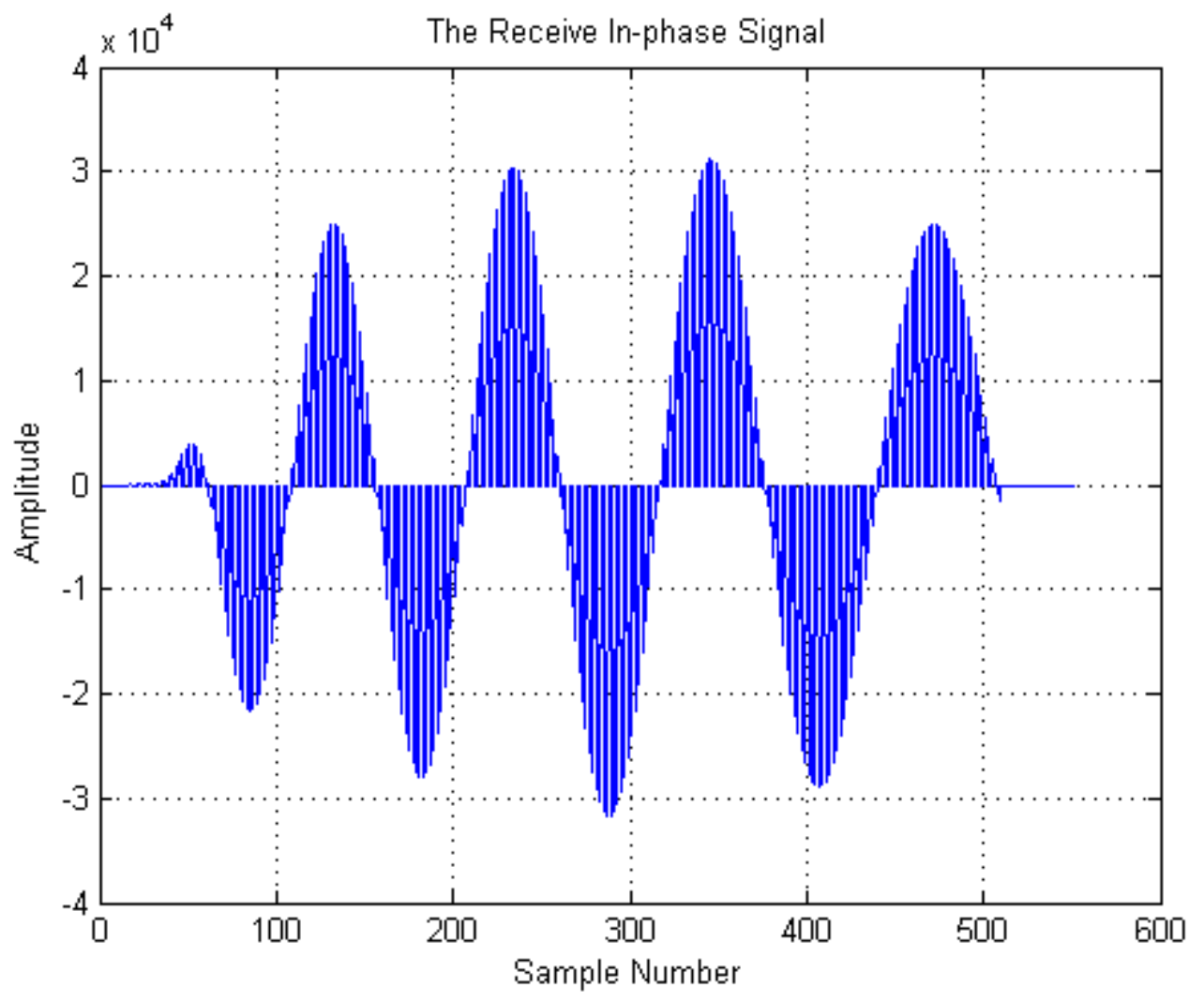

Figure 43: I RX Signal. 


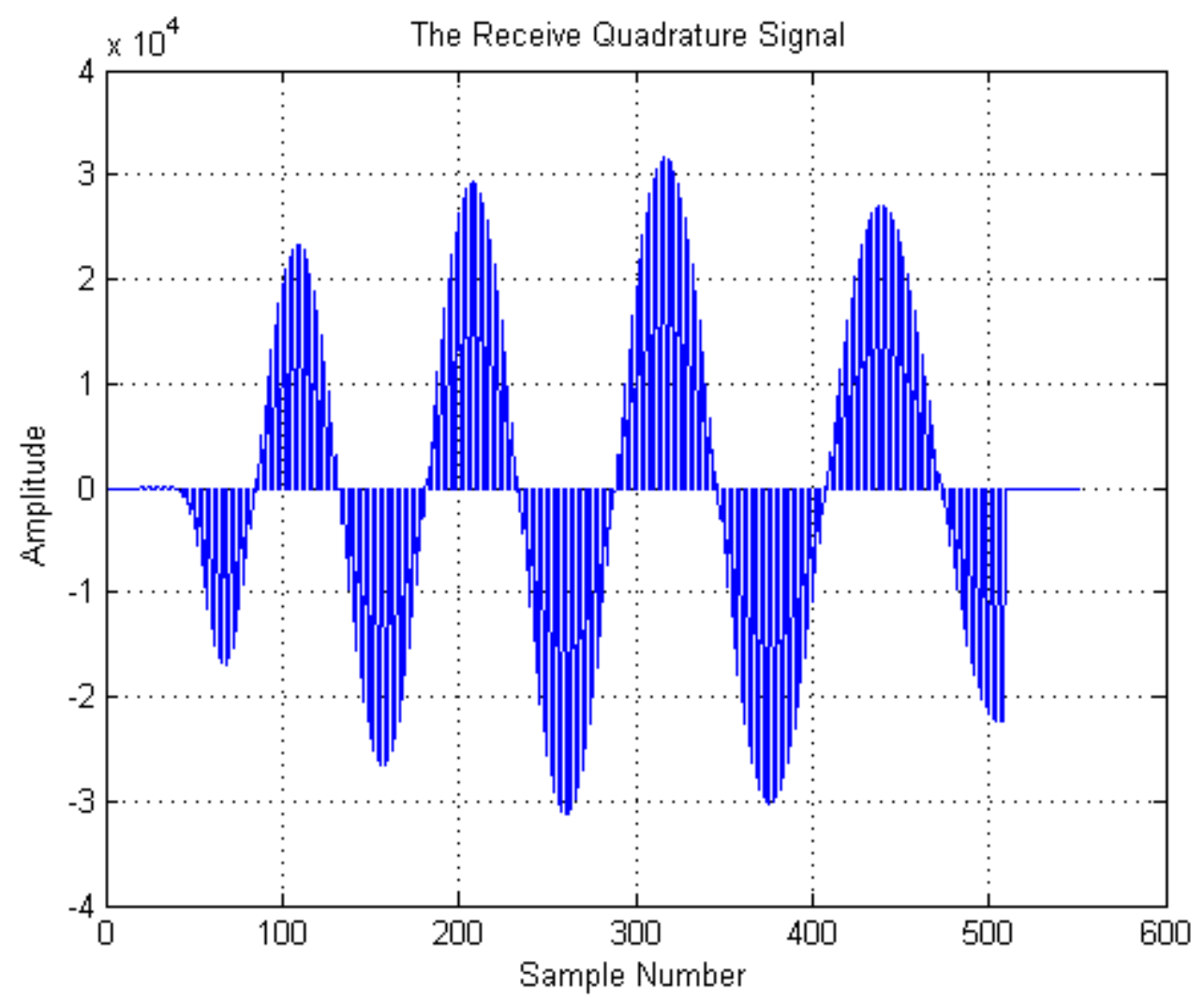

Figure 44: $Q R X$ Signal. 
The mixed TX and RX will then go through a low-pass filter with $50 \mathrm{MHz}$ cut-off frequency. After filtering, the signals will get decimated by 2, to go from $200 \mathrm{MHz}$ to $100 \mathrm{MHz}$. Since the system clock is $200 \mathrm{MHz}$, the signals have to go through FIFO for short time storage then read out to get the decimated signals synchronized with the rest of the FPGA blocks. Figure 45, Figure 46, Figure 47, and Figure 48 show filtering results for I Tx, I RX, Q TX, and Q RX respectively

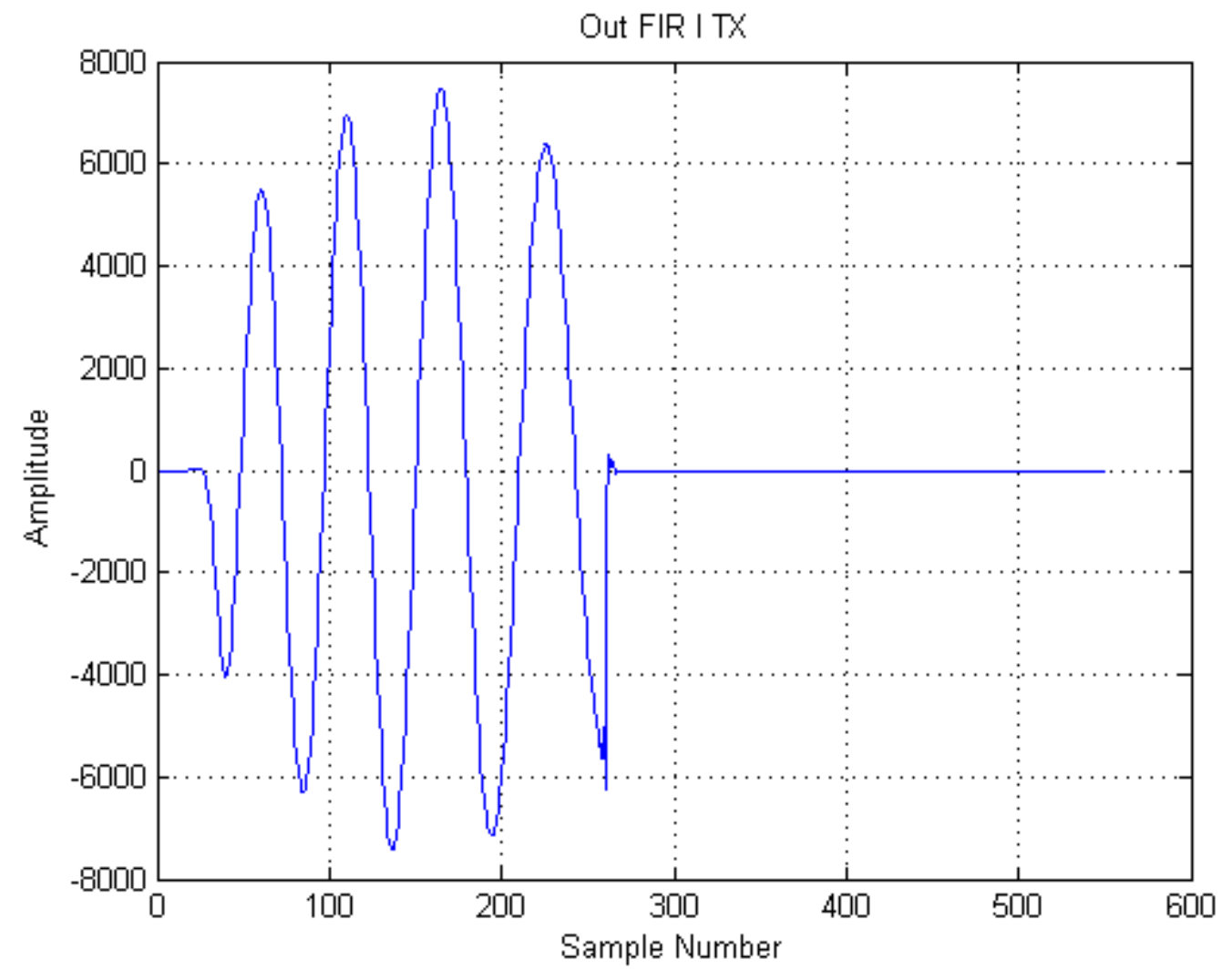

Figure 45: I TX out FIR filter. 


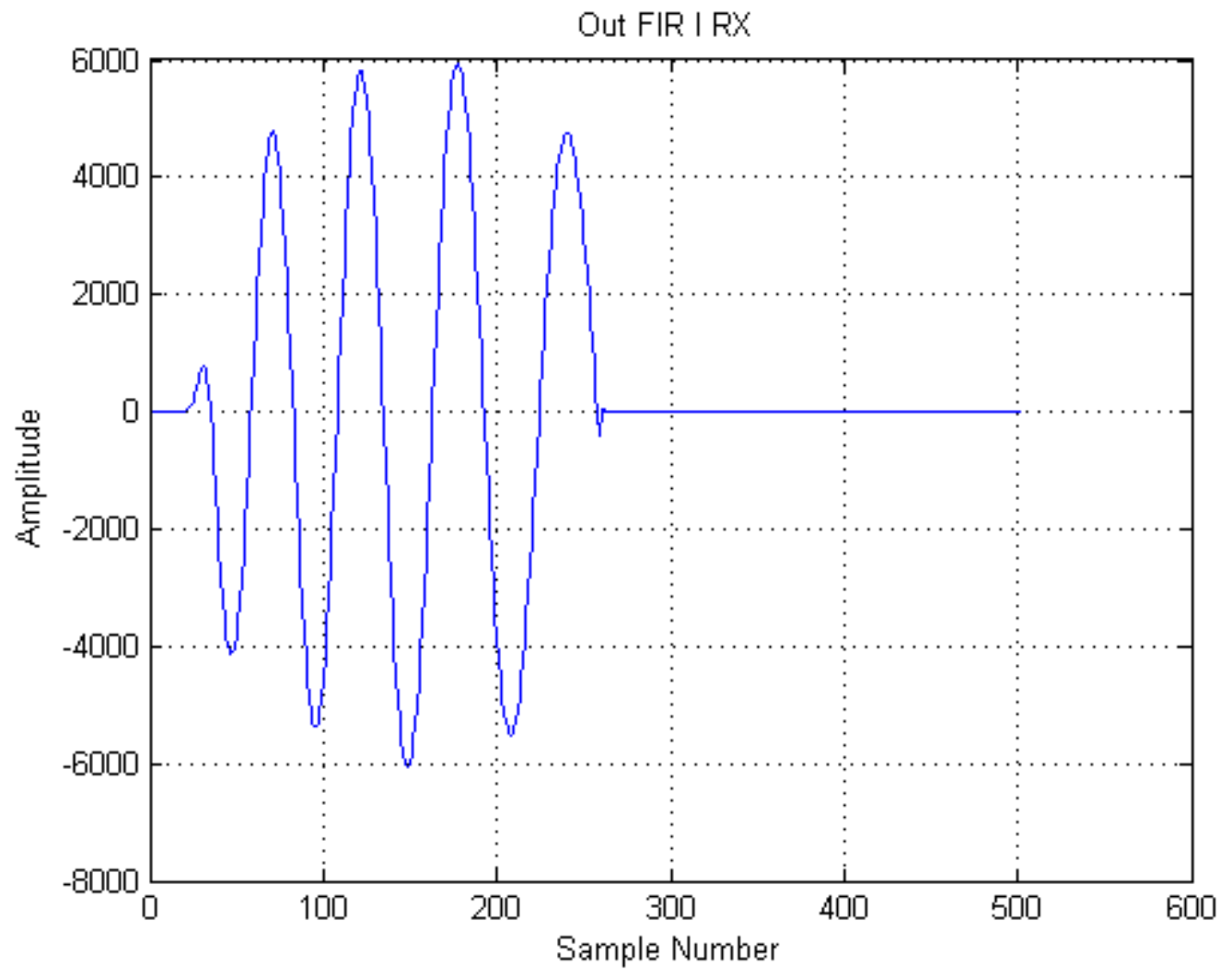

Figure 46: I RX out FIR filter. 


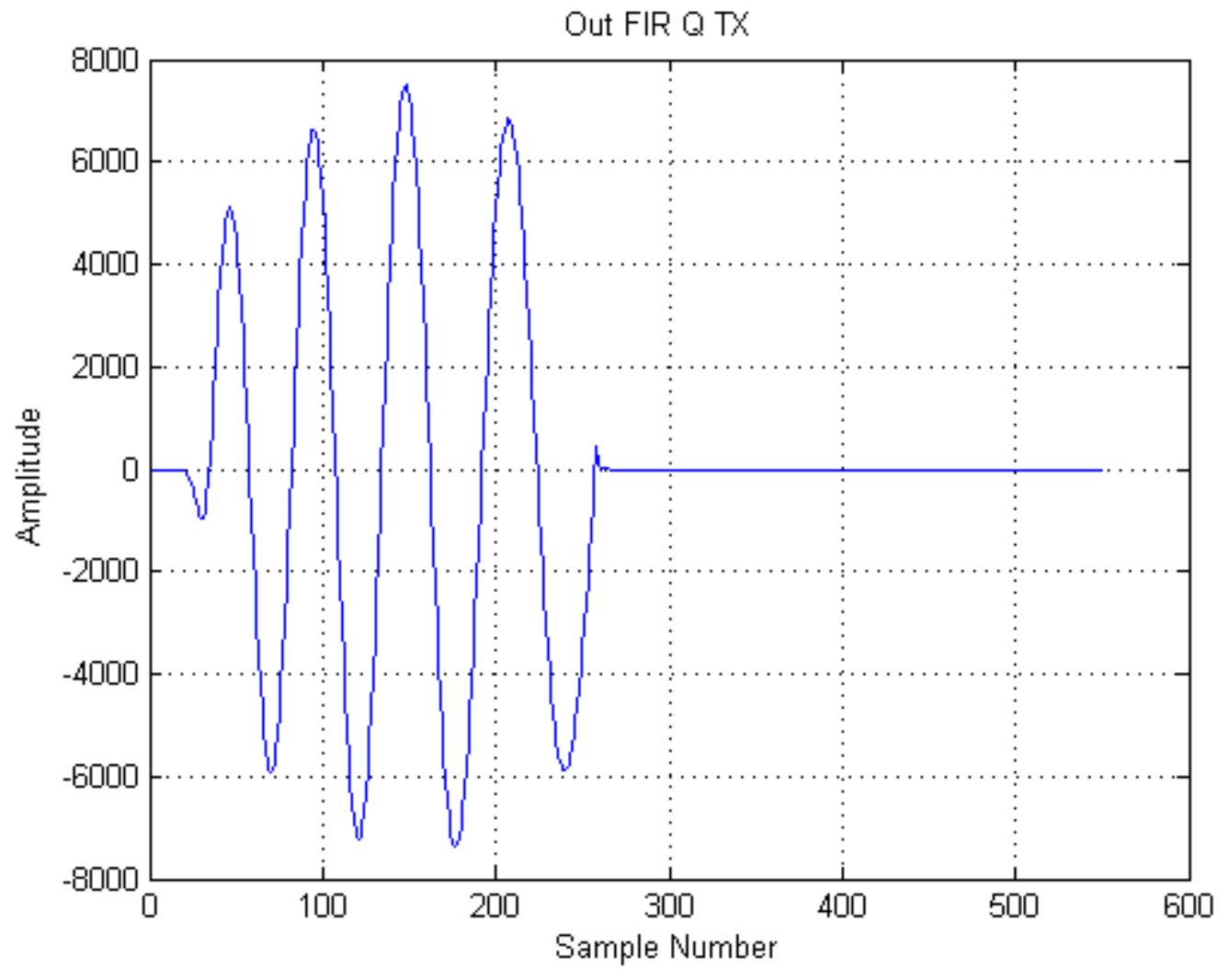

Figure 47: $Q$ TX out FIR filter. 


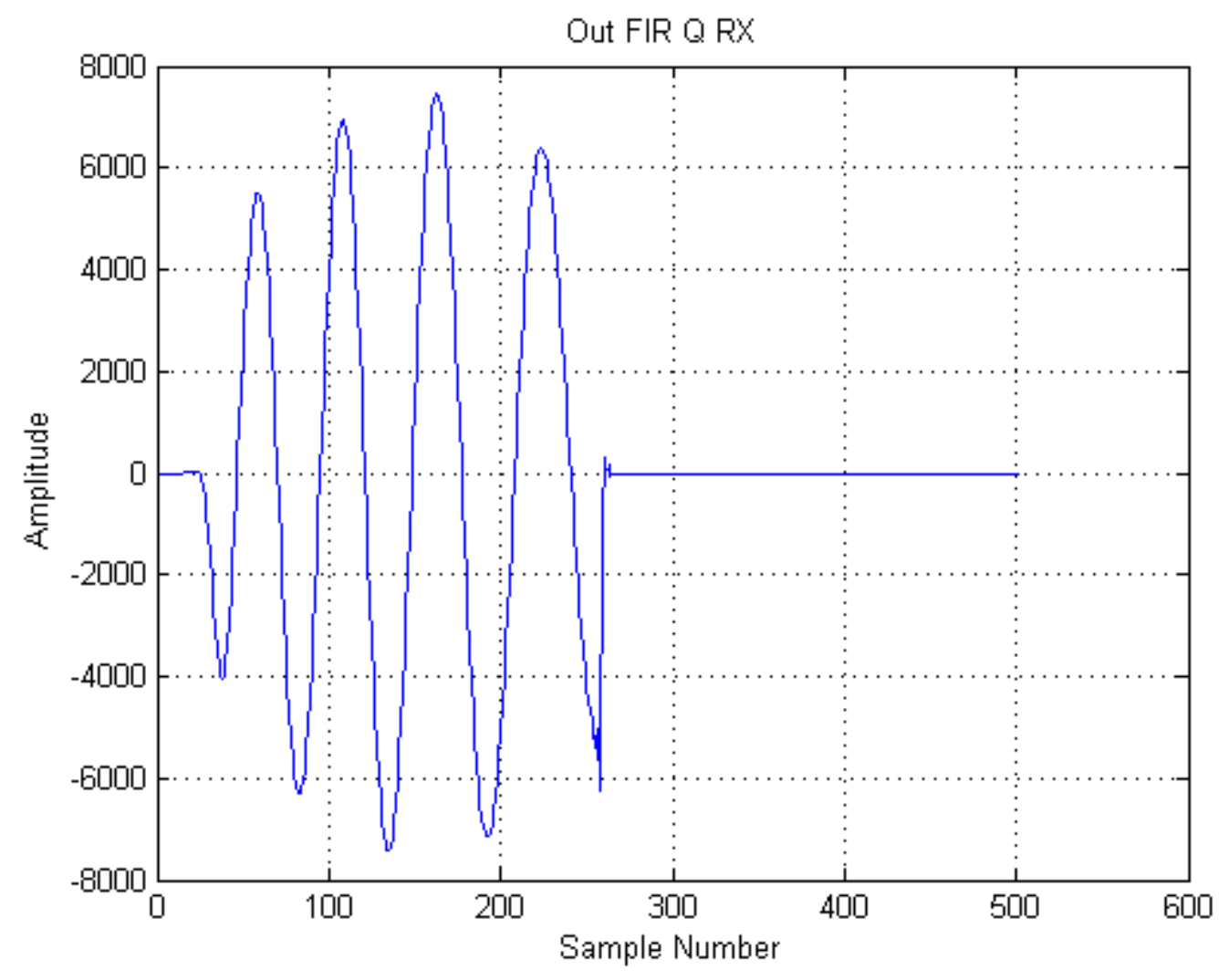

Figure 48: $Q R X$ out FIR filter.

The filtered TX and RX signals will then go through FFT block to go from the time domain to frequency domain for matched filter (cross-correlation). After these two signals are converted to the frequency domain, the result will then complex multiplied by each other. We know that the multiplication in the frequency domain is equal to convolution in the time domain. Figure 49, Figure 50, Figure 51, and Figure 52 show FFT results for I Tx, I RX, Q TX, and Q RX respectively 


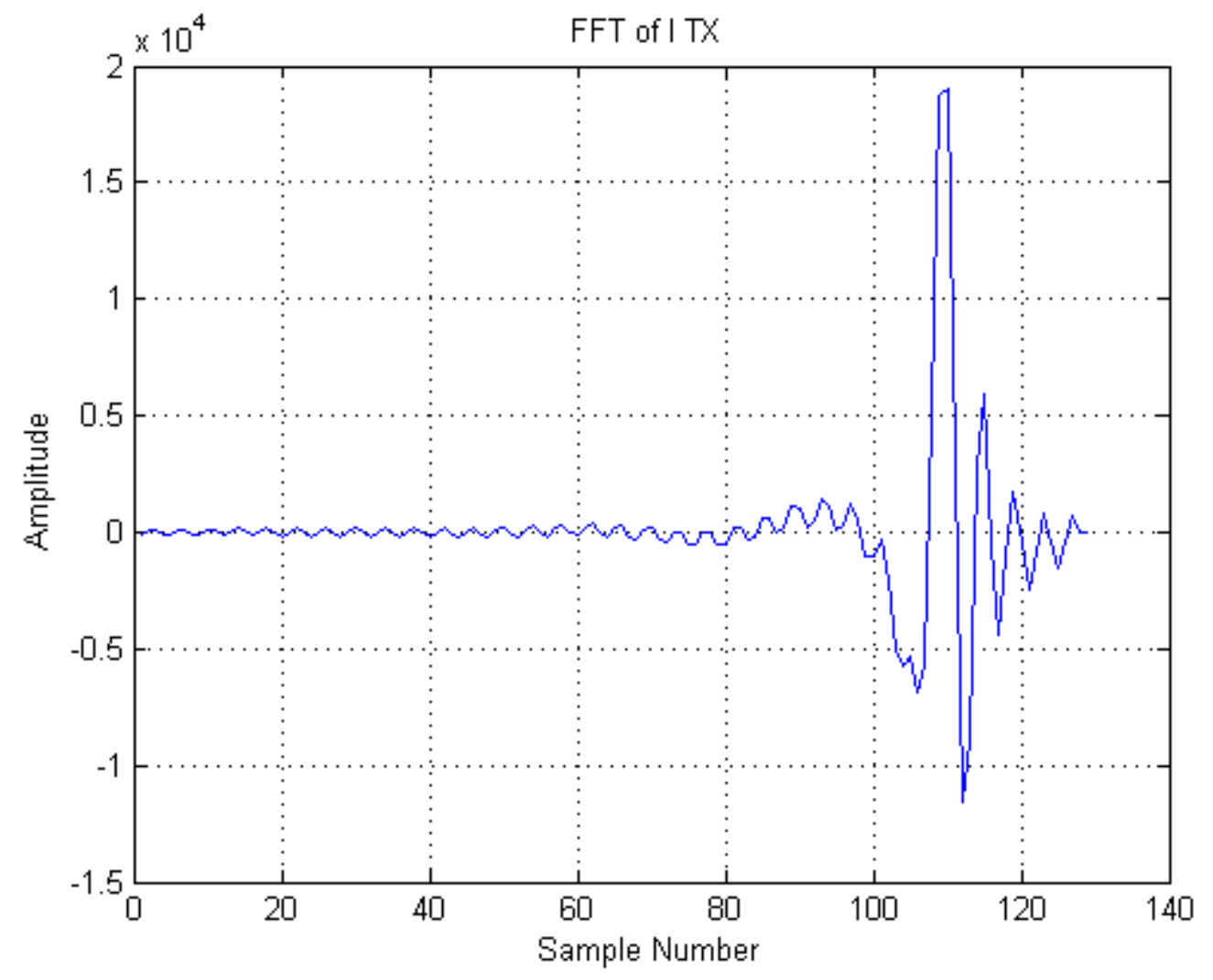

Figure 49: I TX out FFT block. 


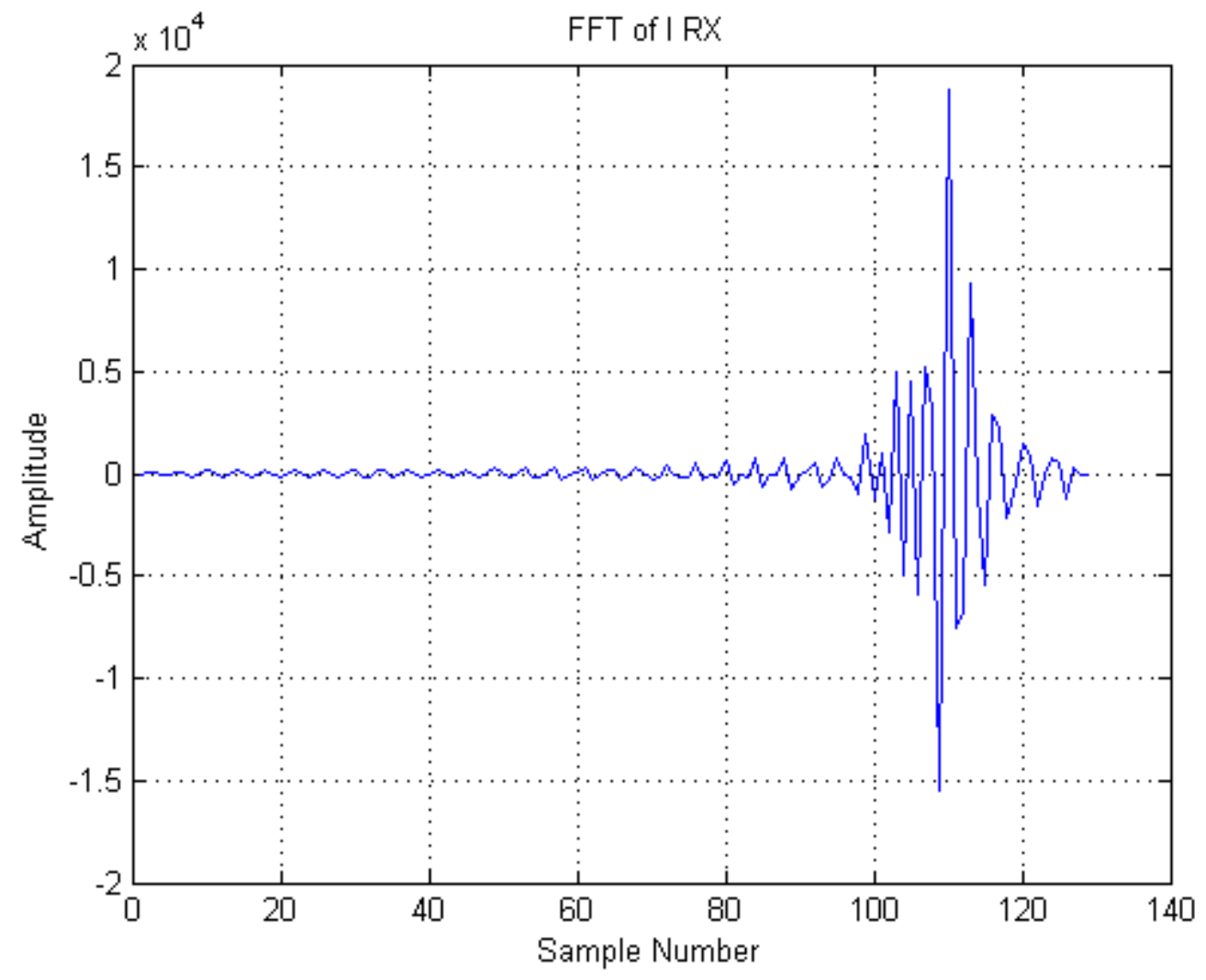

Figure 50: I RX out FFT block. 


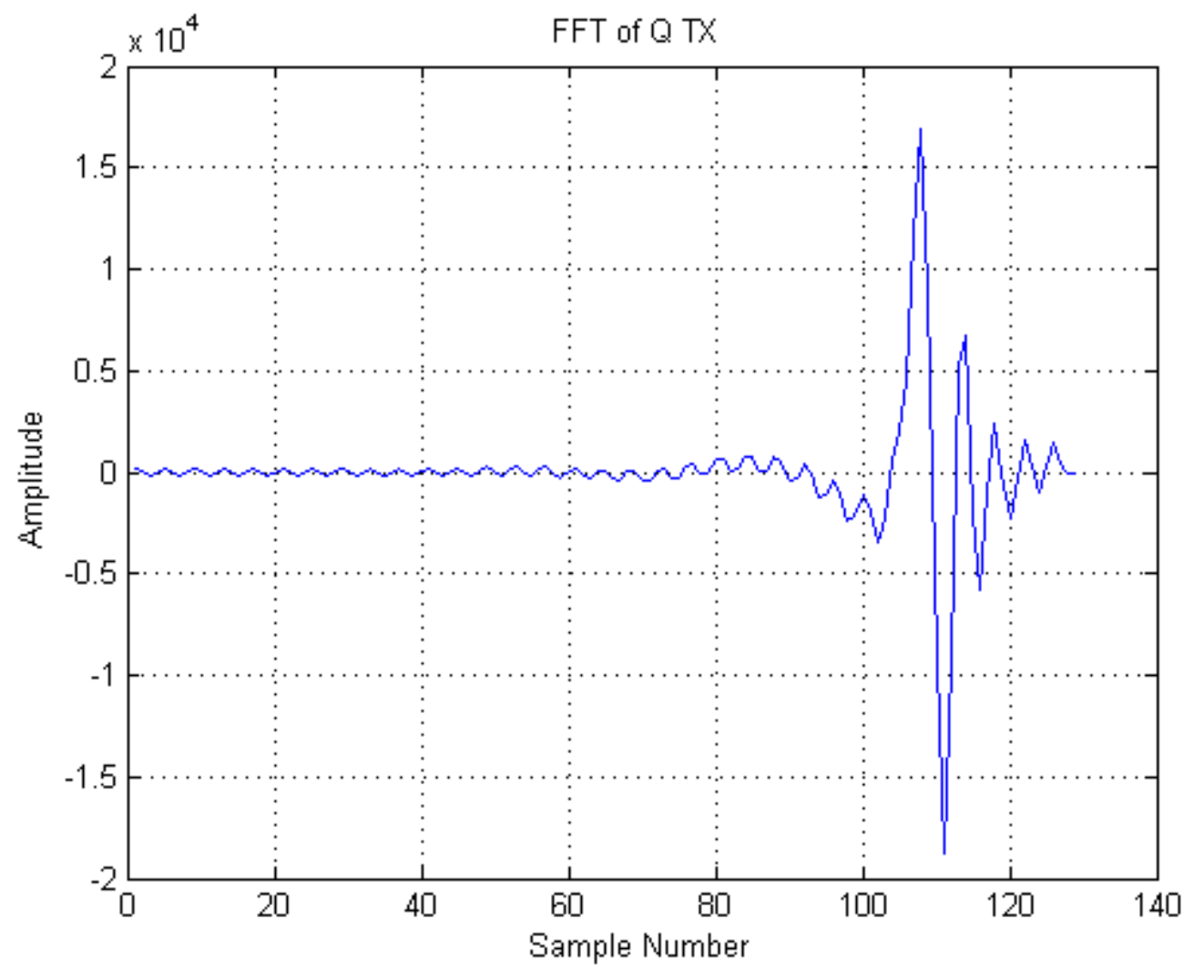

Figure 51: Q TX out FFT block. 


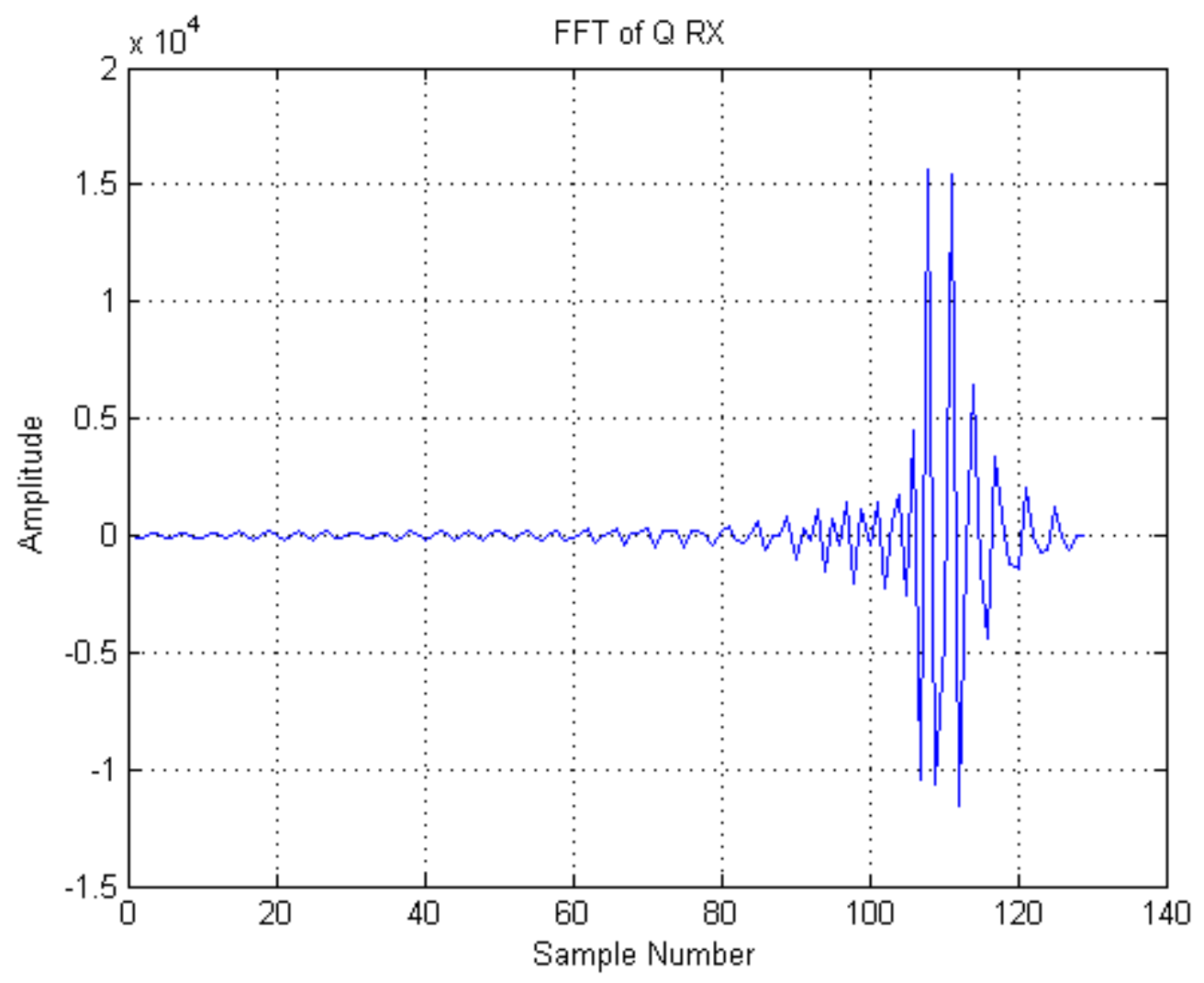

Figure 52: Q RX out FFT block. 
The next step is complex multiply I TX by I RX and Q TX by Q RX. Figure 53 and Figure 54 show complex multiplier results for I Tx by I RX and Q TX by Q RX respectively.

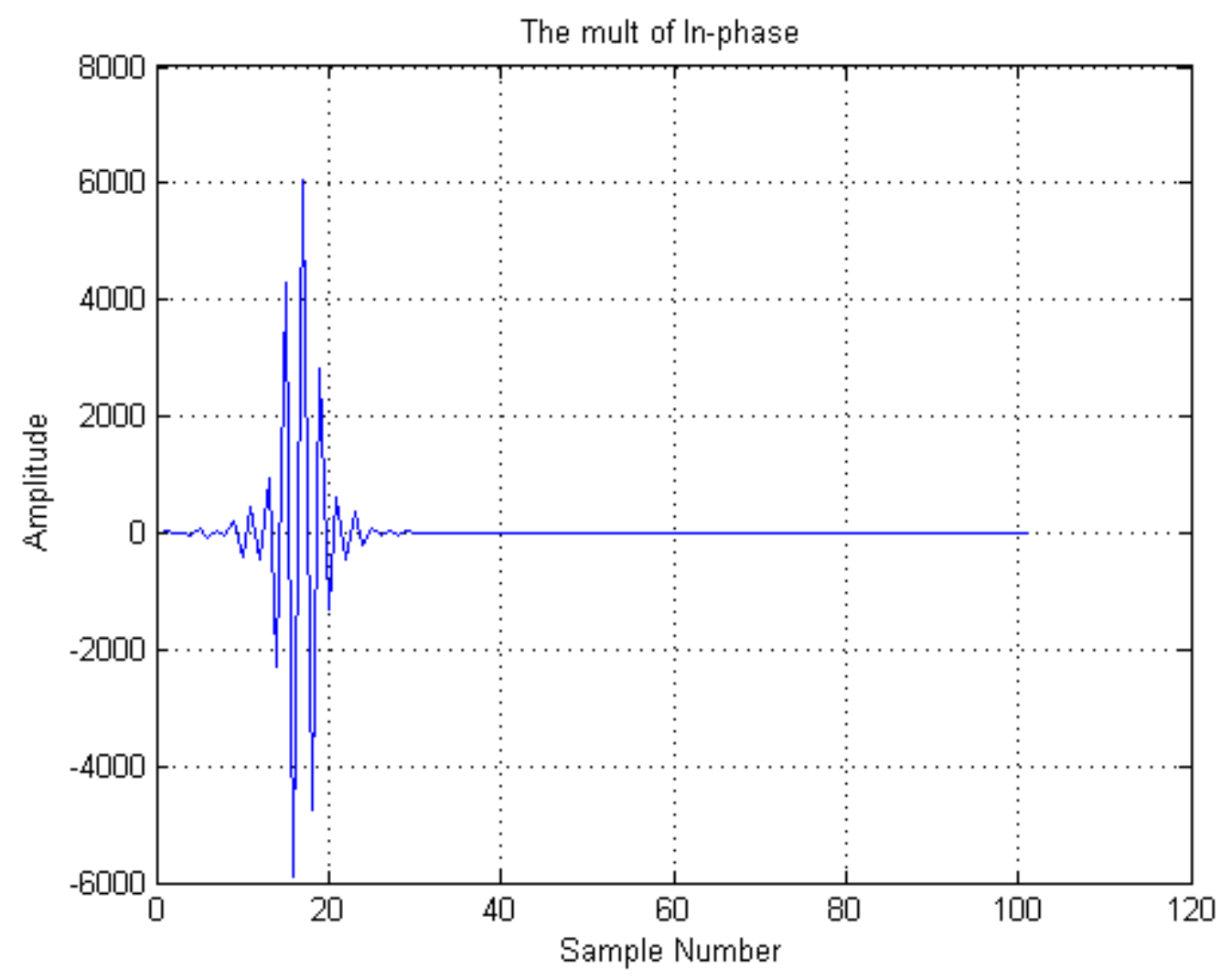

Figure 53: I signal out of the complex multiplier. 


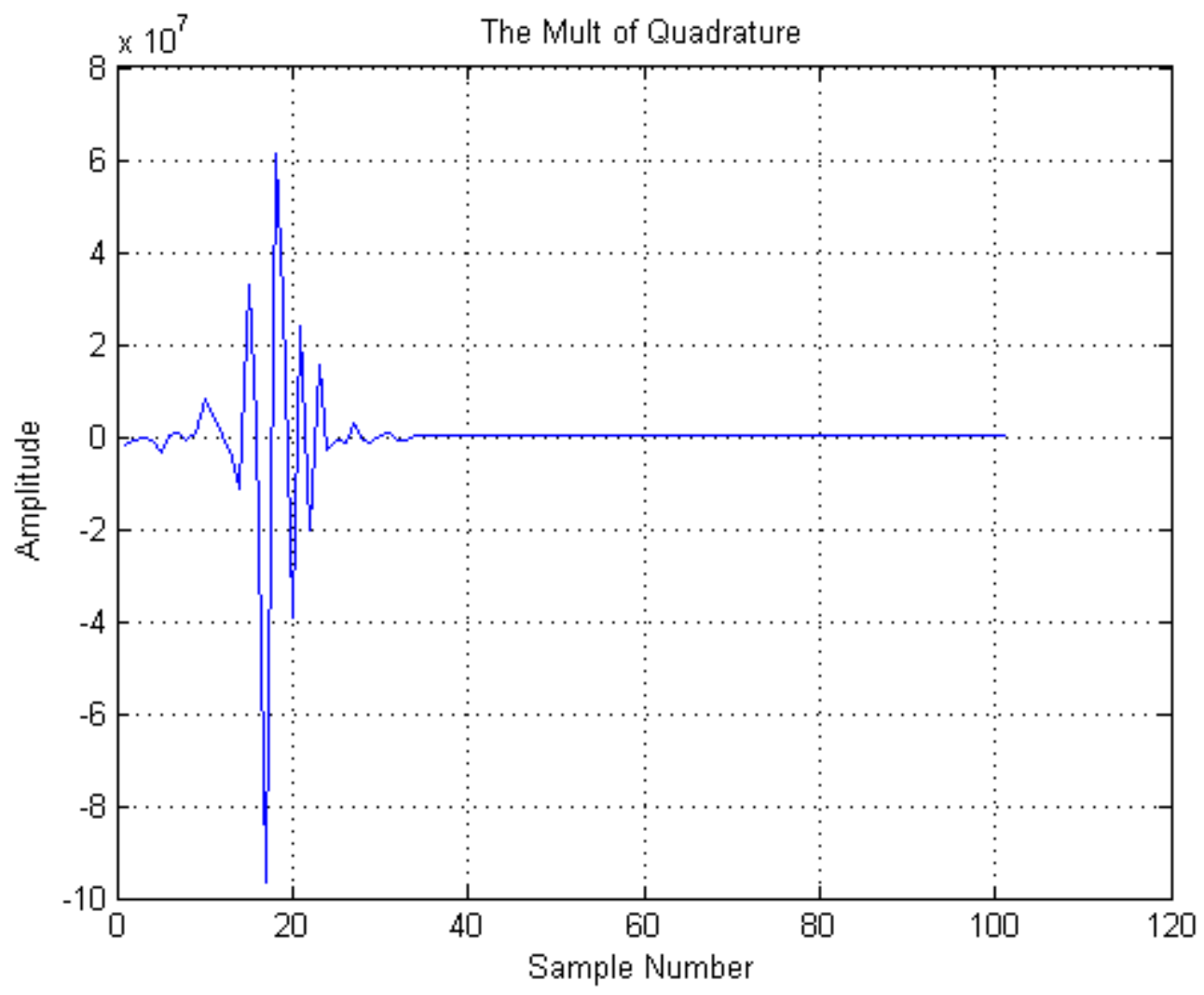

Figure 54: Q signal out of the complex multiplier.

The next step is to IFFT the result of the complex multiplier to convert the signals from the frequency domain back to the time domain. The result of the IFFT is the crosscorrelation between the TX and the RX signal.

Figure 55 and Figure 56 show the results of the IFFT, 


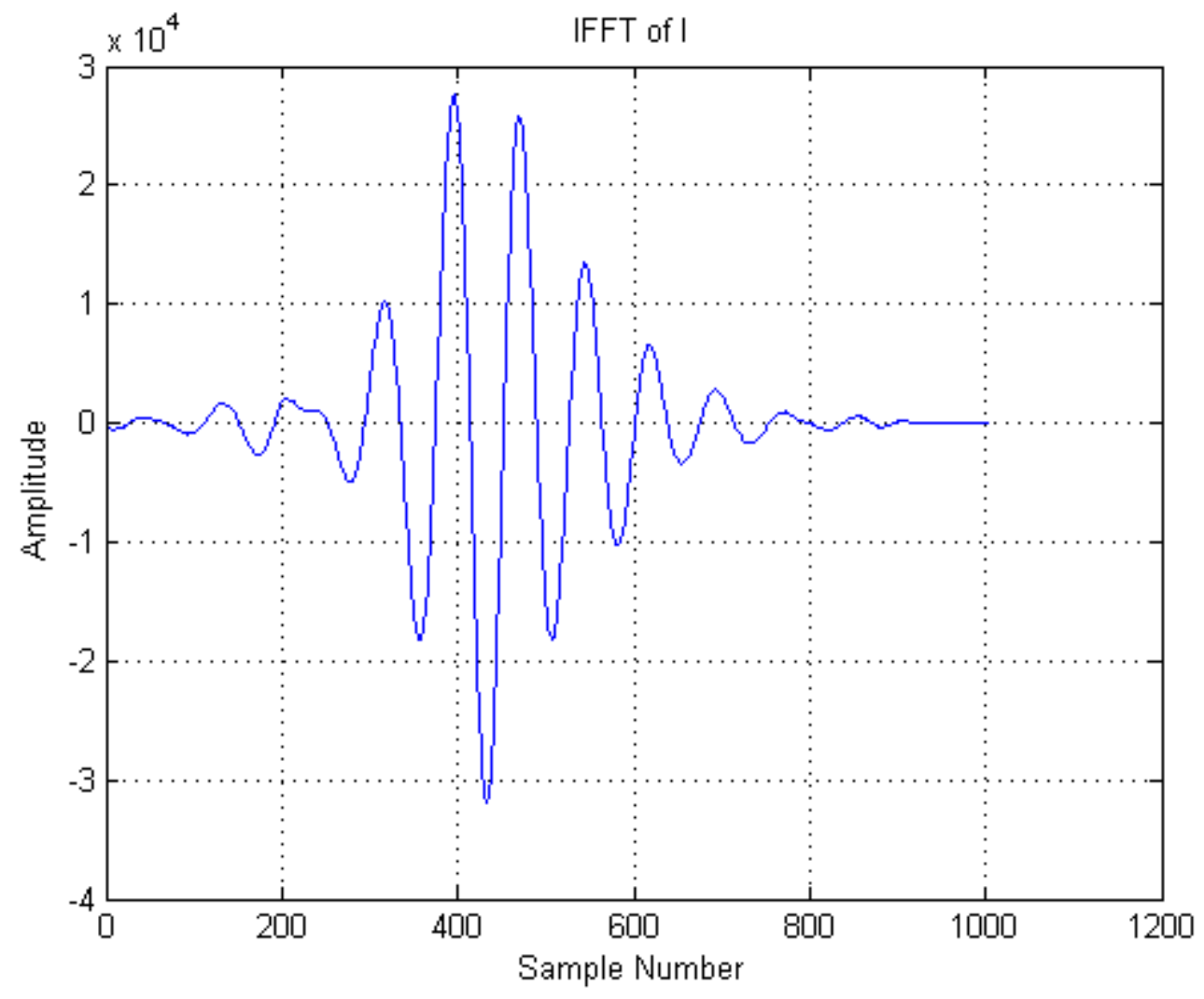

Figure 55: FFT to I signal that is out of the complex multiplier. 


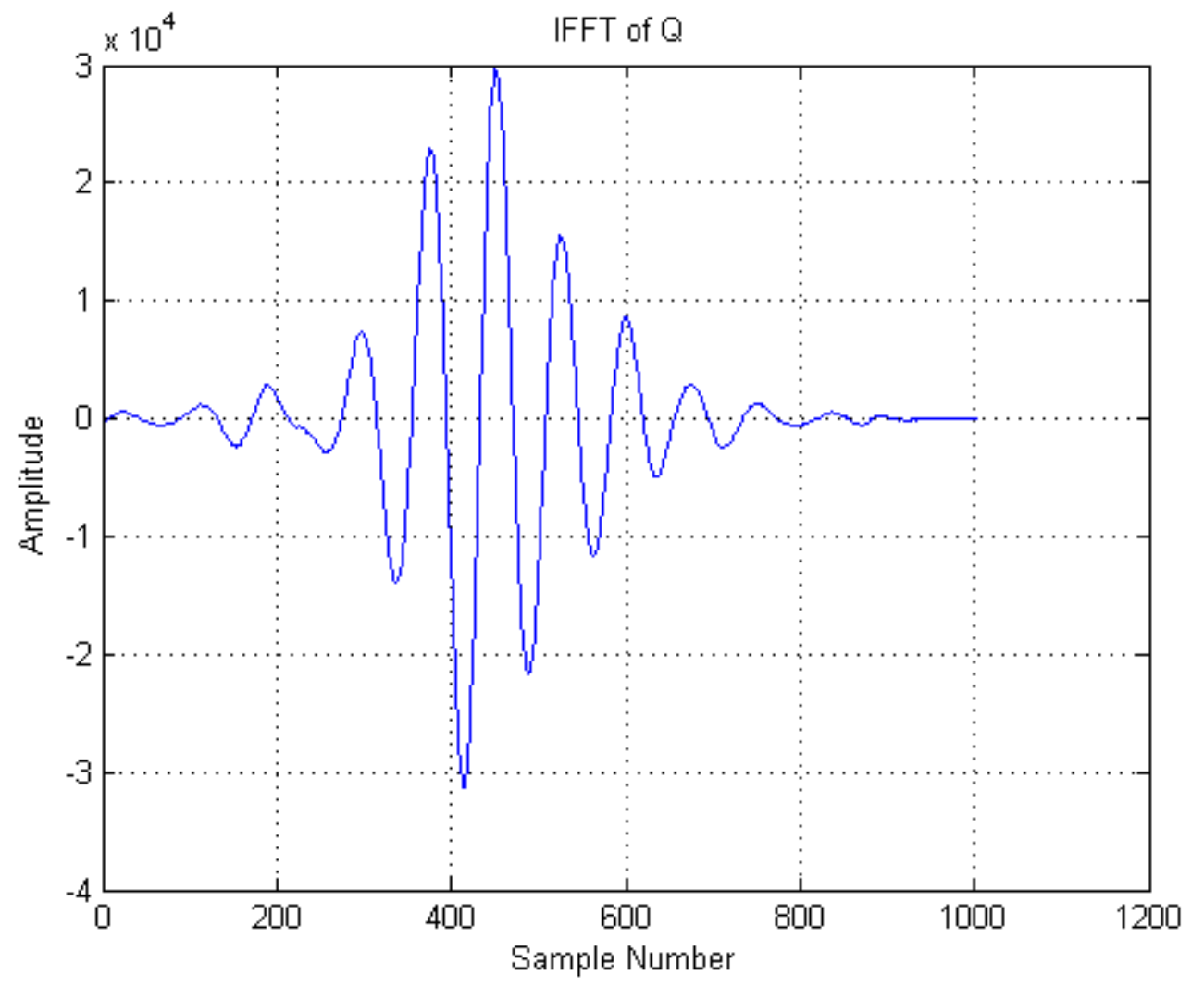

Figure 56: FFT to $Q$ signal that is out of the complex multiplier. 


\subsection{Results}

Figure 57 shows 3D plot of the matched filter's output.

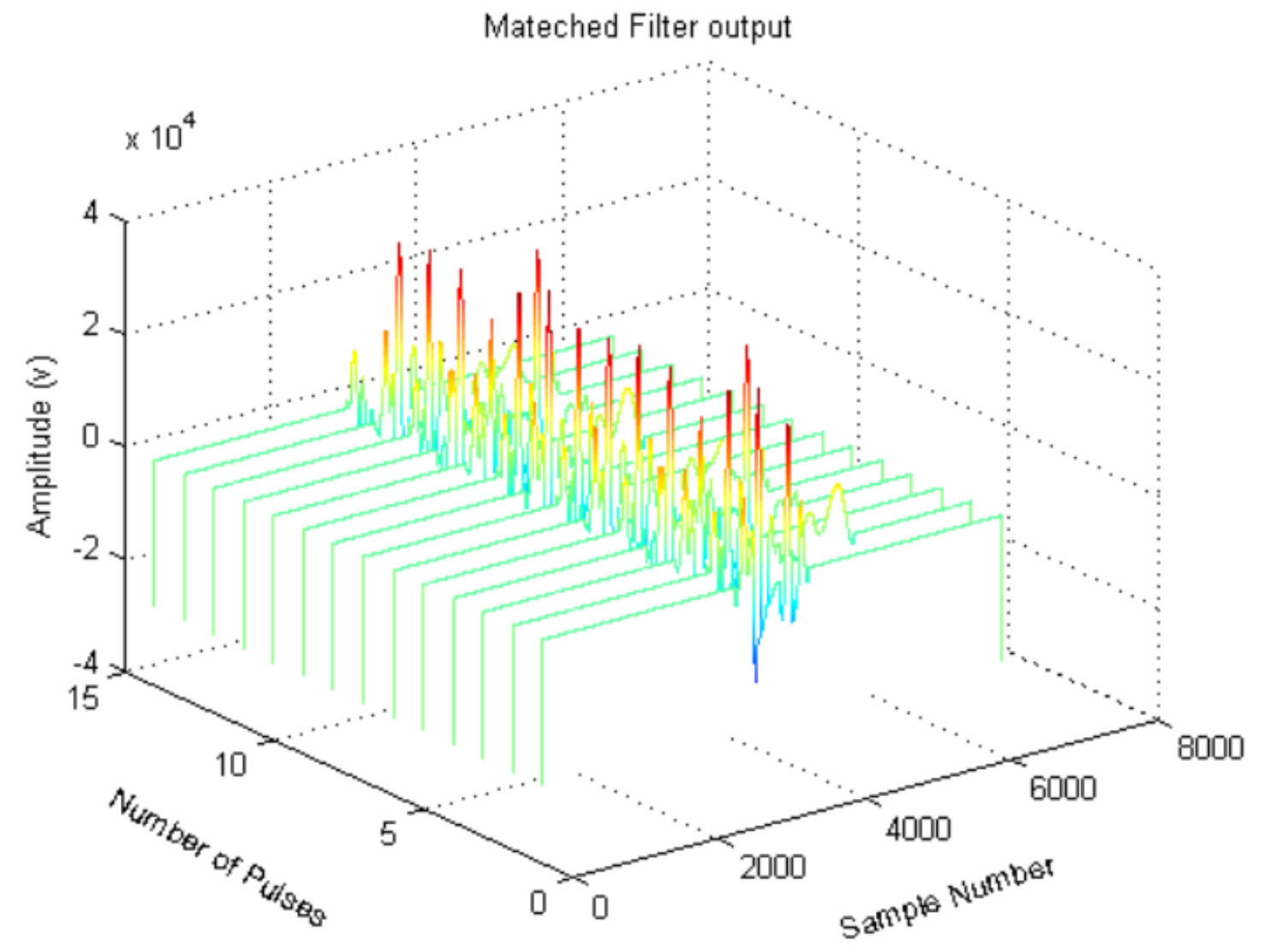

Figure 57: Output of the Matched Filter

The output of the IFFT will get put into 2D matrix (fast and slow time). Now we FFT the in the row of the matrix to get the Doppler frequency $\left(D_{f}\right)$.

The result of the Doppler range is plotted in Figure 58 and Figure 59. 


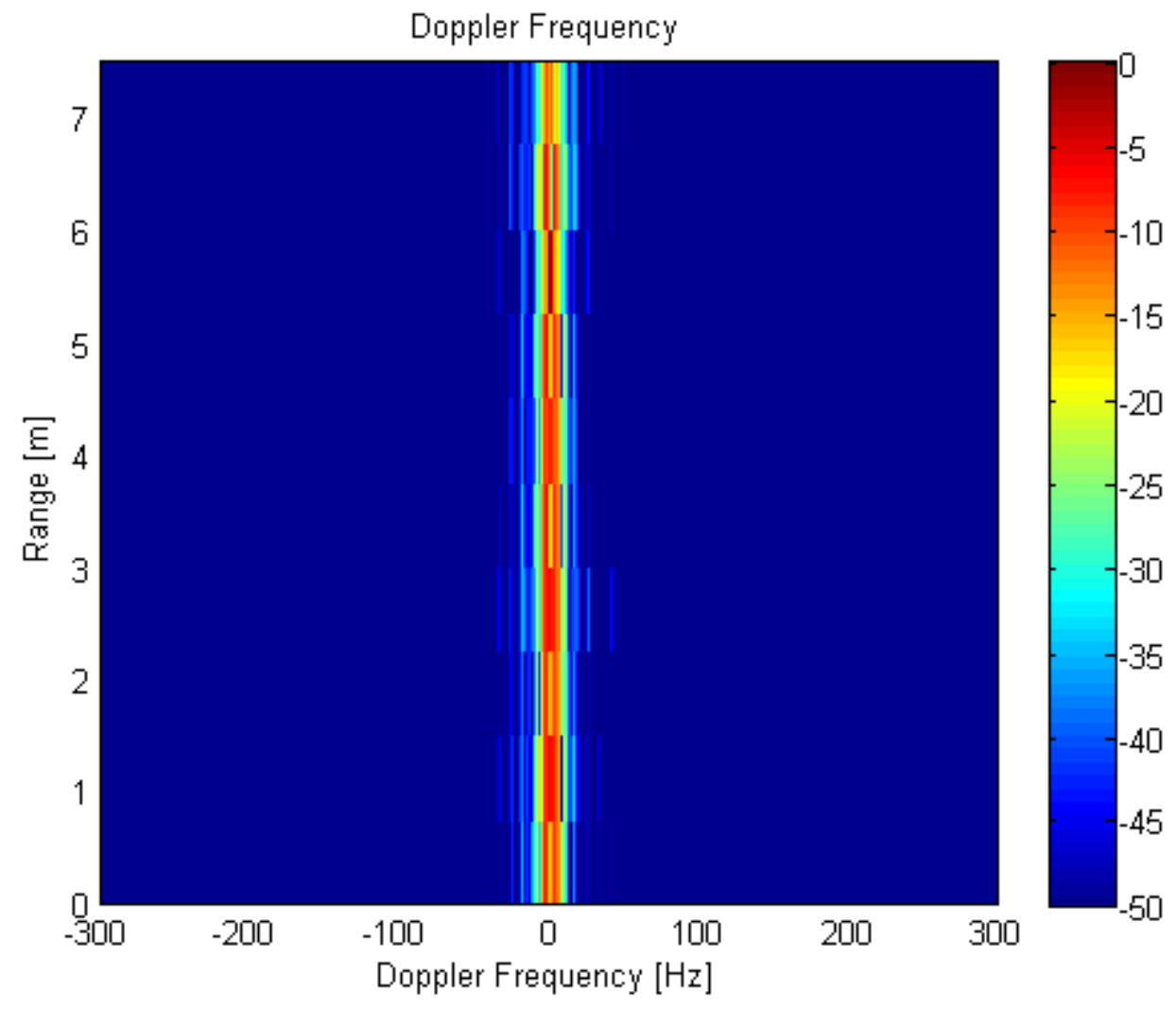

Figure 58: Range-Doppler Image Result of the FPGA design. 


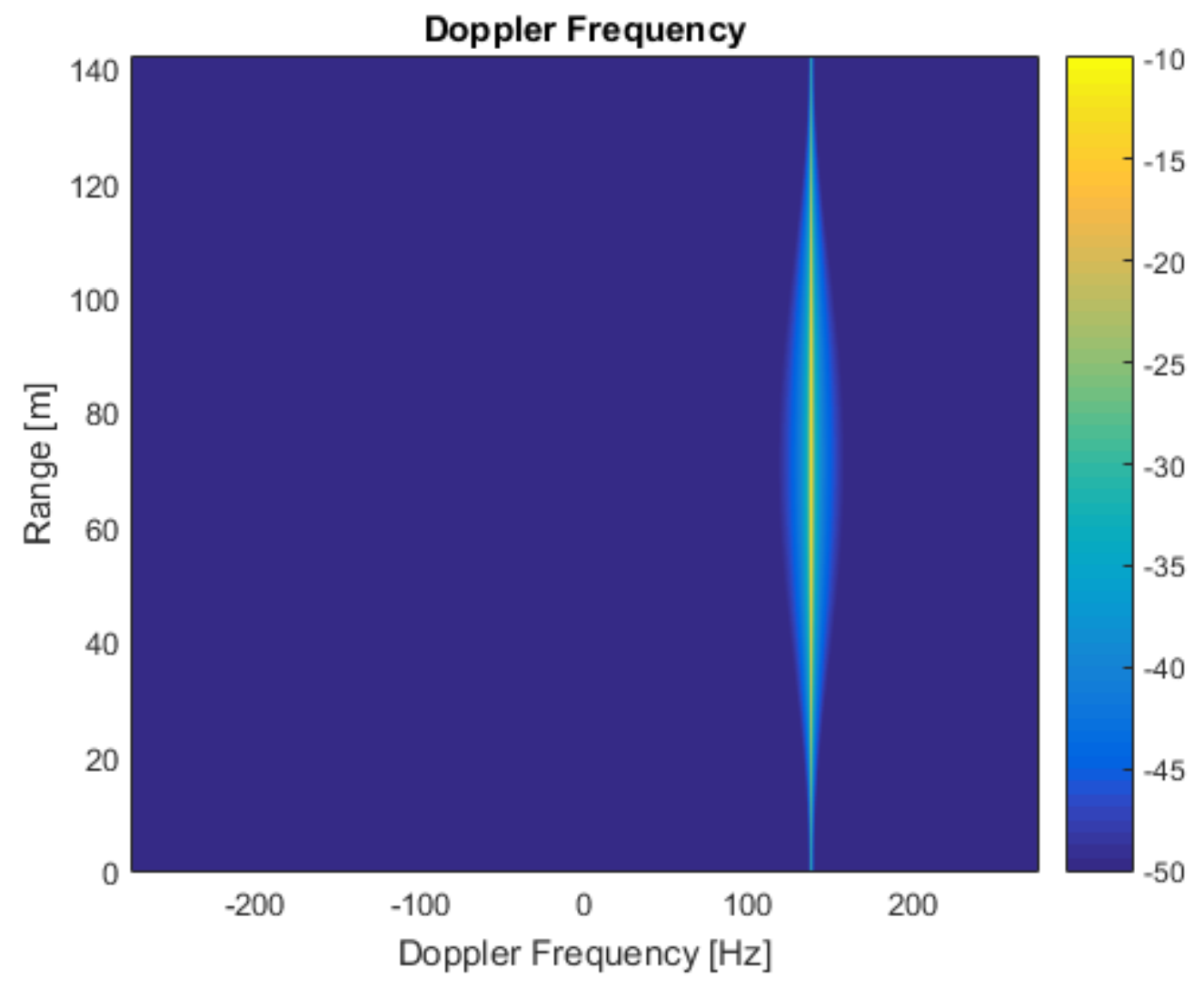

Figure 59: One more example of Range Doppler. 


\section{CHAPTER 7}

\section{CONCLUSION}

Marine radars are reliable and affordable, but they only detect the range of the targets but not the velocity. So the concept of applying digital-coherent processing to data collected by magnetron based navigation radar has been demonstrated.

This research is to sample and coherently process measurement data collected via a magnetron oscillator based marine radar system. Such radars operate non-coherently, and as such, offer limited surveillance in clutter rich environments. That's mean the Doppler frequency is unknown, so, the speed of the target is unknown too. For that reason, we used FPGA technology to sample and process the received data for Doppler shift analysis. We have produced a design to modify the marine radar hardware and capture the transmit and the receive signals. We process the captured data on FPGA boards. Matched-Filter and FFT/IFFT analysis for Doppler decomposition is used to compute the velocity of moving targets. Also, in our research, we are improving the affordability of edge-of-the-art marine radars by using the cutting-edge FPGA technology to improve performance. Modern sensors and systems use FPGA technology instead of microprocessors or microcontroller. Finally, we will coherently integrate two or more marine radars for bi-static operation. 


\section{BIBLIOGRAPHY}

[1] Furuno US webpage

http://furunousa.com/products/productdetail.aspx?product=1623\&

category $=$ Products $\% 20 \% 3 \mathrm{~A} \% 20$ Radars $\% 20 \% 3 \mathrm{~A} \% 20$ Radars

[2] J.P.Y Maa and H.K. Ha, "X-band radar wave observation System," Project report. Minerals Management Service. US Department of the Interior, 2005.

[3] L. Pedersen, I. Zawadzki, N.E. Jensen, and H. Madsen, "Assessment of QPE results from $4 \mathrm{~kW}$ x-band local area weather radar (LAWR) evaluated with s-band radar data," The Fifth European Conference on Radar in Meteorology and Hydrology, 2008.

[4] N.E. Jensen, L. Pedersen, and H. Skov, "Development and application of x-band radar systems for automatic detection and registration of migrating birds," 34tl' Conference on Radar Meteorology, 2009

[5] Lim S. et al. ' Reflectivity retrieval in a networked radar environment". IEEE International Geoscience and Remote Sensing Symposium, 2004

[6] Dolan B. and S. Rutledge. "An integrated display and analysis methodology for multivariable radar data”. AM S 32nd Conference on Radar Meteorology, 2005. 
[7] Bharadwaj N. and V. Chandrasekar. 'Waveform design for casa x-band radars”. AM S 32nd Conference on R adar M eteorology, 2005.

[8] Bharadwaj N. and V. Chandrasekar. 'Waveform design considerations for casa radar network". ERAD, 2006.

[9] Patent US 2009/0315754, Downloaded from:

http://www.freepatentsonline.com/20090315754.pdf

[10] G.E. Smith et al. "High Power Coherent-On-Receive Radar For Marine Surveillance", 2013 International Conference on Radar

[11] Pastorino, M. (2010) Applications of Microwave Imaging, in Microwave Imaging, John Wiley \& Sons, Inc., Hoboken, NJ, USA.

[12] Kristoffersen, S.; Hoel, K.V.; Thingsrud, O.; Kalveland, E.B., "Digital coherent processing to enhance moving targets detection in a navigation radar," in Radar Conference (Radar), 2014 International, vol., no., pp.1-6, 13-17 Oct. 2014 doi: 10.1109/RADAR.2014.7060295

[13] L. Lo Monte, D. Erricolo, F. Soldovieri, M.C. Wicks, "Radio Frequency Tomography for Tunnel Detection," IEEE Transactions on Geoscience and Remote Sensing. Vol 48, No. 3, pp. 1128-1137, Mar 2010.

[14] Wicks, M. C.: RF tomography with application to ground penetrating radars. In Proc. IEEE 41st Asilomar Conference ACSSC 2007, pages 2017-2022, Nov 2007. 
[15] Nicolaisen, H.; Holmboe, T.; Hoel, K.V.; Kristoffersen, S., "High resolution rangeDoppler radar demonstrator based on a commercially available FPGA card," in Radar, 2008 International Conference on , vol., no., pp.676-681, 2-5 Sept. 2008

[16] Hessner, K.; Hanson, J.L., "Extraction of coastal wavefield properties from X-band radar," in Geoscience and Remote Sensing Symposium (IGARSS), 2010 IEEE

International , vol., no., pp.4326-4329, 25-30 July 2010 